\title{
Exploration of Narrative Structure in Games for Story \\ Creation
}

by

Bethany Dunfield

A thesis submitted to the Faculty of Graduate and Postdoctoral Affairs in partial fulfillment of the requirements for the degree of

Masters of Information Technology
in

Digital Media

Carleton University

Ottawa, Ontario

(C) 2017

Bethany Dunfield 


\section{Abstract}

While video games have become a popular medium for storytelling, as a more subjective form of game content, evaluation of written or generated narratives remains difficult. We propose a model for game narrative (the combination of story and its discourse) that can be used to both help write game stories, and evaluate existing ones. This model is based on scholarly models of narrative, alongside narrative structuralism, which can give information about each narrative event and its' position within the overall sequence of events making up the story. We also show from the literature that strong stories feature an external goal or quest which can only be achieved by a character resolving their own internal struggles.

To create our model, we performed a study of existing video game stories, using the aggregate critical score as our metric for quality. Stories were broken down into their component events, and data was logged about each event's importance, whether it sourced internally from a character or externally, and whether the event corresponded to a component of narrative macrostructures such as "The Hero's Journey". Results from our analysis show a strong relationship between the ratio of internal "character-driven" events versus external "plot-driven" events and a story's quality. We were also able to make recommendations about overall story event structure, such as limiting strings of external, or "plot-driven", events. 


\section{Acknowledgements}

I would like to dedicate this thesis to the late Dr. Anthony Whitehead, who was my thesis supervisor and mentor until his death. There were many times through the course of my graduate studies that I considered quitting. But I left every meeting with him inspired and ready to take on the new challenges of the week. Without his wisdom and guidance, I truly do not feel I would be writing this document.

I would also like to thank Dr. Ali Arya, who stepped in to fill Anthony's shoes. Coming into a project in its late stages, and having no knowledge of what we had done before is a difficult situation. However, Ali worked hard to make sure the adjustment went as smoothly as possible. My thanks extend to the entire administration and faculty of the School of Information Technology, who went above and beyond to make the transition for us students as easy as they could, despite dealing with the loss of a valued colleague and friend. 


\section{Table of Contents}

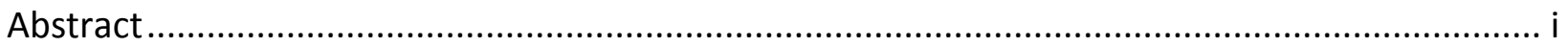

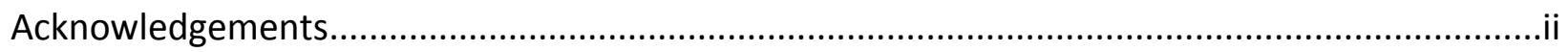

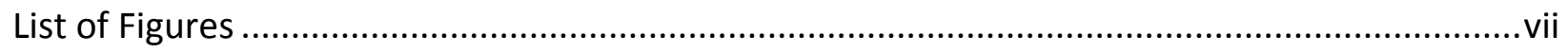

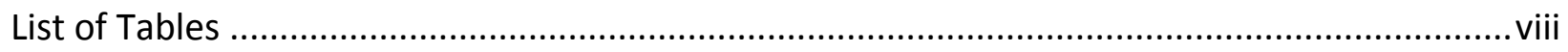

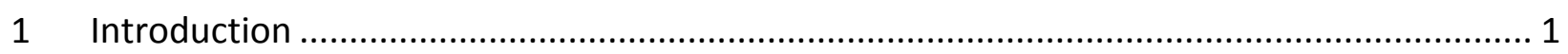

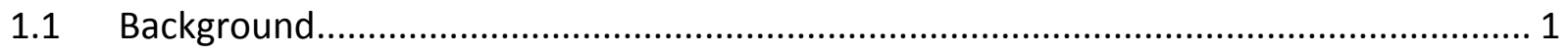

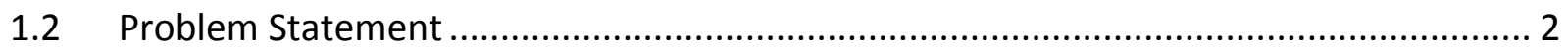

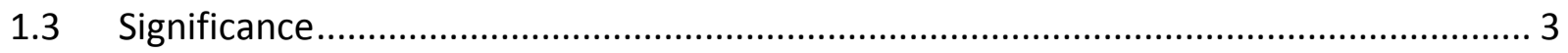

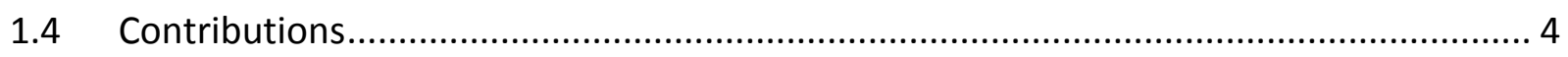

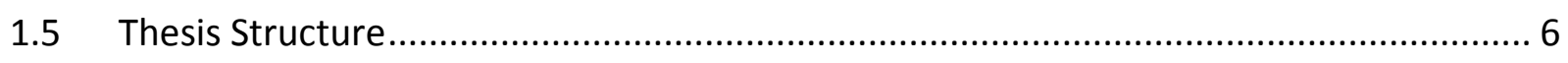

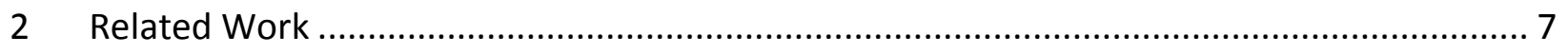

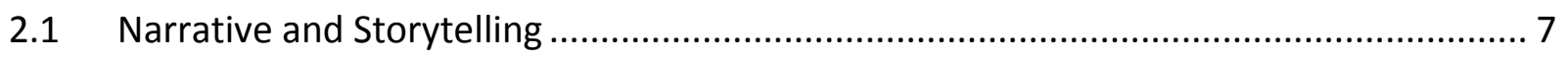

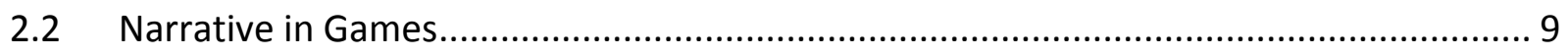

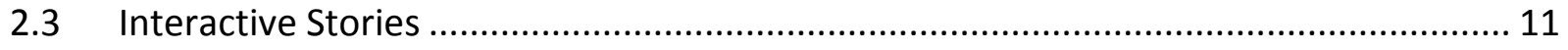

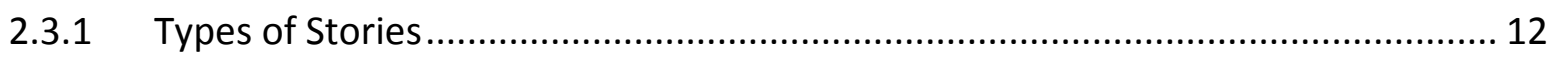

2.3.2 Player versus character ..................................................................... 13

2.3.3 Embedded and Emergent Spectrum ..................................................... 14

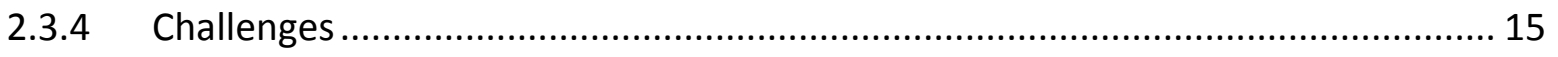

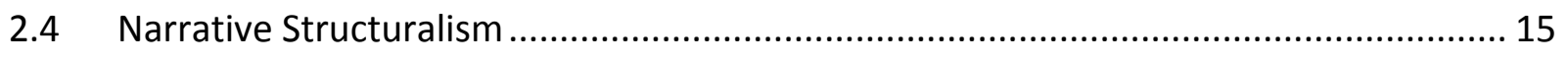

2.5 Procedural Content Generation Overview....................................................... 18 
2.5.1 Narrative Generation

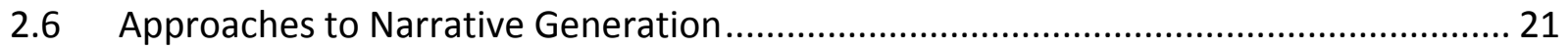

2.6.1 Emergent Narratives / Player-Driven..................................................... 22

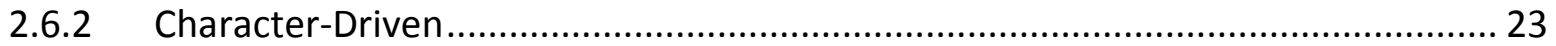

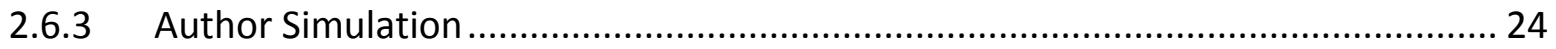

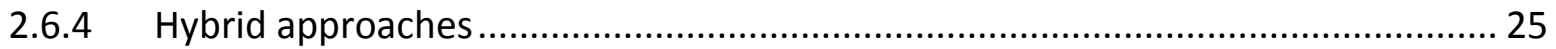

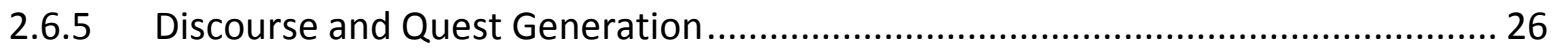

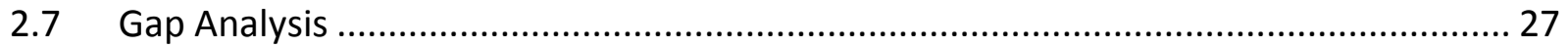

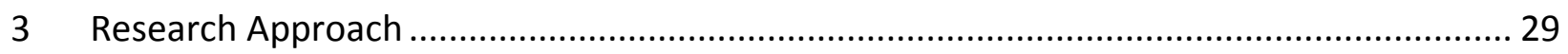

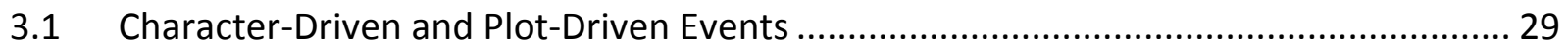

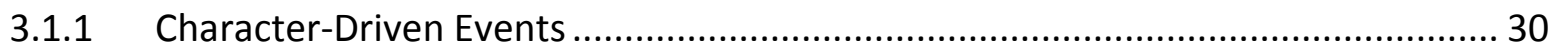

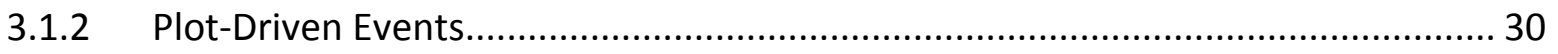

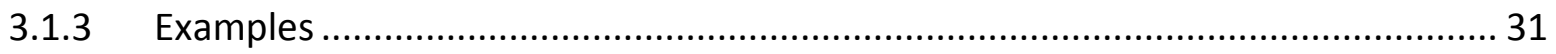

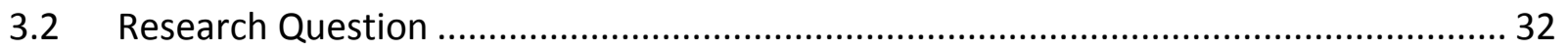

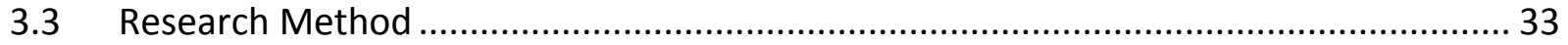

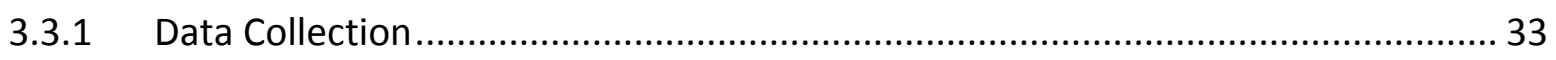

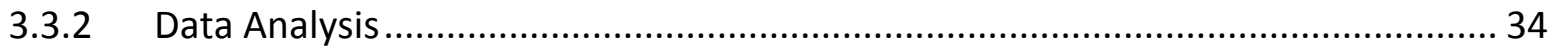

3.3.3 Visualizer Development for Preliminary Analysis ....................................... 37

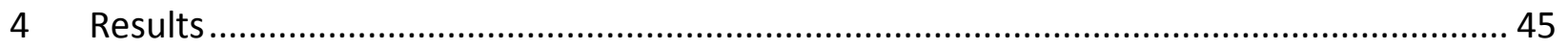

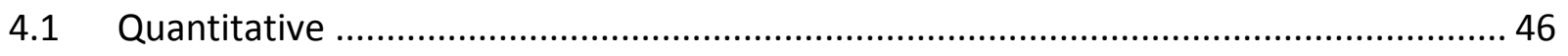


4.1.1 Overall Results ................................................................................................ 46

4.1.2 Character-Driven and Plot-Driven Probability Analysis .......................................... 48

4.1.3 Narrative Macrostructures …………………................................................ 54

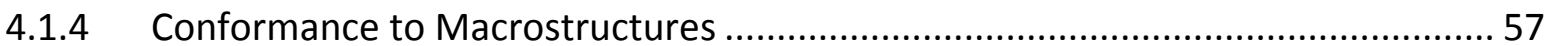

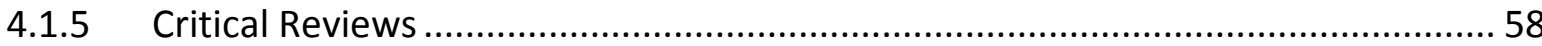

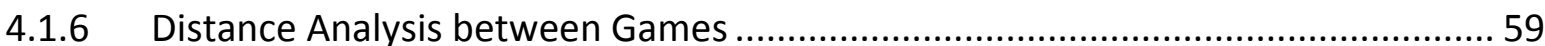

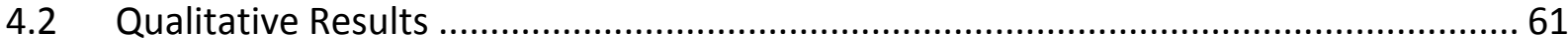

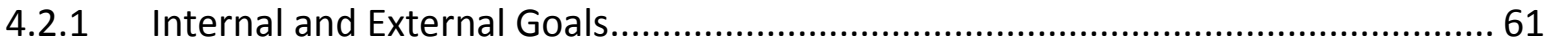

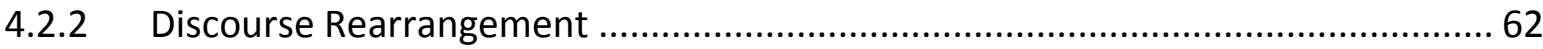

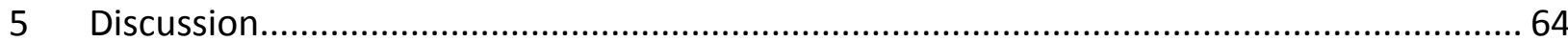

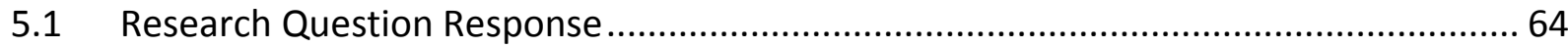

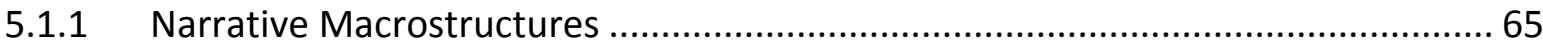

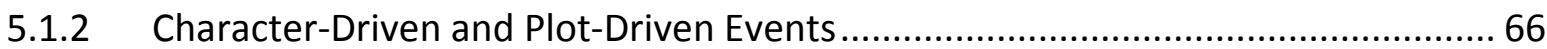

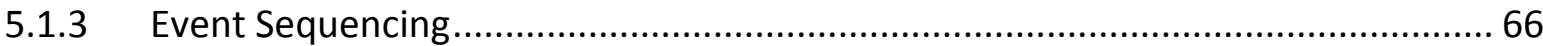

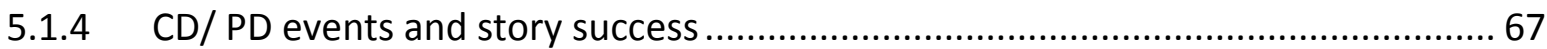

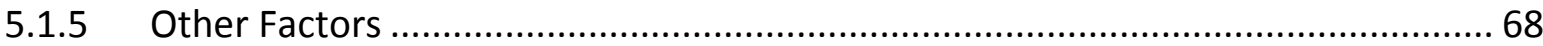

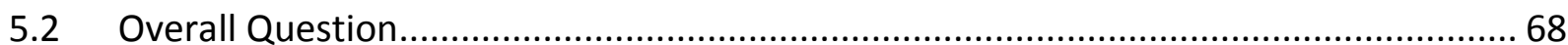

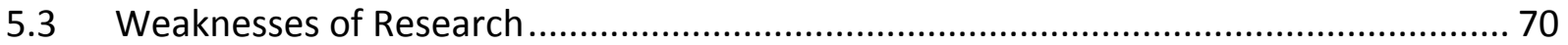

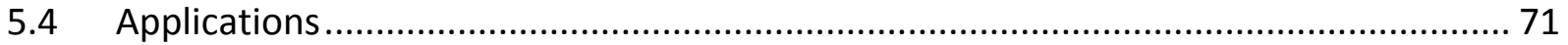

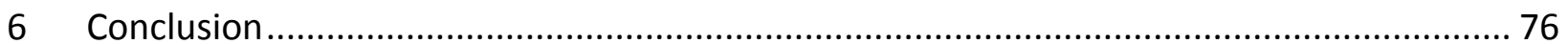




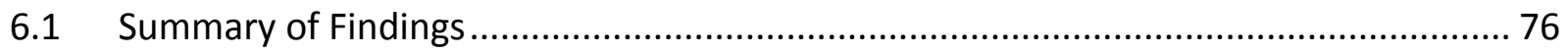

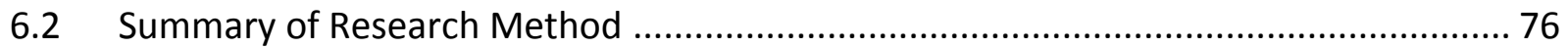

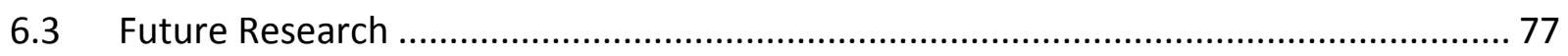

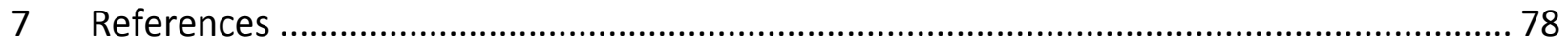

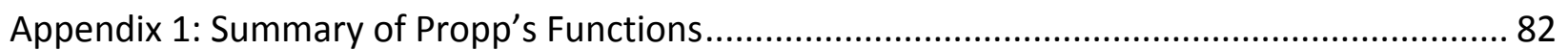

Appendix 2: Narrative Macrostructure Equivalency Comparison ......................................... 83

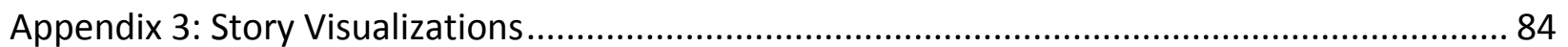

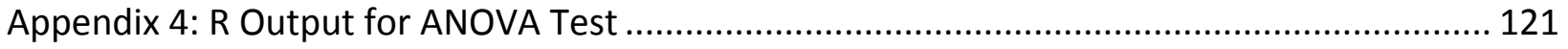

Appendix 5: Character Internal / External Goal Analysis..................................................... 123 


\section{List of Figures}

Figure 1. Graphical representation of a linear story..................................................... 12

Figure 2. Branching and Hypertext sample structures .................................................... 13

Figure 3: Narrative Visualizer interface for the Arc and Character Interactions visualizations ... 38

Figure 4: Narrative Visualizer interface, showing the game selection list .............................. 39

Figure 5. Arc Visualization of the story from "The Last of Us" video game. ............................ 40

Figure 6. Character Interactions Visualization for Game Stories........................................ 41

Figure 7: The Arc and Character Interactions visualizations overlaid .................................. 42

Figure 8. Character Co-occurrence Matrix for the story of "Metal Gear Solid 3"..................... 43

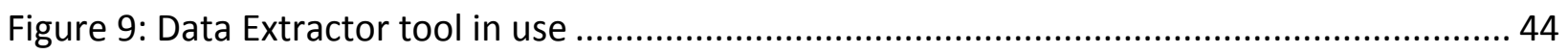

Figure 10. Percentage of Character-Driven events against Game Score............................... 46

Figure 11. Percentage of kernel events which are Character-Driven versus game score ............ 47

Figure 12: Markhov State Machine for 2-length story event strings ..................................... 49

Figure 13: Markhov State Machine for 3-length story event strings. ................................... 52

Figure 14: Number of PP-P Event Strings plotted against Game Score................................ 54 


\section{List of Tables}

Table 1. Sample Data Sheet for "The Legend of Zelda: Ocarina of Time".............................. 35

Table 2 . Listing of analysed games and their aggregate critical scores................................... 45

Table 3. Analysis of Variance of the count of CD and PD events, related to the game Score..... 47

Table 4. Summary of Median and Mean scores when the data set is split............................. 48

Table 5. Observed Probability values for 2-tuple event strings ........................................... 50

Table 6. Chi Squared Comparison of the Median and Third split datasets for 2-event strings ... 50

Table 7. Observed Probability values for 3 -tuple event strings ............................................. 51

Table 8. Binomial p-values for 3-tuple event strings. All Binomial tests were "Greater". .......... 51

Table 9. Chi Squared Comparison of the Median and Third split datasets for 3-event strings ... 53

Table 10. The Hero's Journey Structural Data within the Dataset .......................................... 55

Table 11. The Writer's Journey Structural Data within the Dataset......................................... 55

Table 12. Structural Data for Propp's Functions within the Dataset..................................... 56

Table 13. Hamming Distance Matrix for Mid-Truncation....................................................... 61

Table 14. Number of positive and negative story opinions within critical reviews. ................. 59

Table 15. Summary of Discourse Rearrangement styles found in our data..............................63 


\section{Introduction}

\subsection{Background}

Narrative and storytelling are complex structures and activities that have been well studied throughout history. A common model for stories features a person who faces an internal struggle (which may or may not be known to them). This person overcomes external challenges which force them to confront and grow past their internal struggles in order to accomplish their ultimate goal [1][2]. Another important aspect of narrative is the concept of structure. Structuralist approaches break down narrative into its component parts and analyze the properties of each part at a variety of levels [3]. This allows for in-depth analysis of each event in the story and understanding how they come together to create a complete narrative.

A more common application of narrative structuralism is in what we term in this research "narrative macrostructures". These structures outline an entire story from start to finish and describe the key events within the story to get from the beginning to the end. The Hero's Journey [4] is a well-known macrostructure for adventure stories. Other examples include Propp's 31 functions describing the fairy tale [5], and Vogler's adaptation of the Hero's Journey: The Writer's Journey [6].

In this work, we study stories as they relate to video games, as a new storytelling medium. Videogames are capable of supporting complex narratives, as well as both linear and non-linear story structures. However, the narrative structure in video games, while significant in their success, has not been properly studied. 


\subsection{Problem Statement}

Game development costs have increased as technological capabilities and team sizes have grown [7]. This can lead to a higher risk of failure should the final game not be a success. And, as costs rise, the metric for success rises as well, with games often not turning a profit unless they are top sellers [8]. Stories, while not necessary for games [1], can make a game more attractive to players [9]. Stories are a subjective domain though, which makes them difficult and potentially costly to evaluate [10].

Procedural Generation of game content is one way to mitigate development costs [11]. Many forms of procedural generation have seen widespread industry adoption. Tools such as SpeedTree which create vegetation are used in many large titles today [12]. Generation of random levels has created entire genres of gaming, such as the roguelike [13]. While these types of generation are relatively common in commercial games, story generation remains rare outside of academic circles. Researchers have also frequently identified major deficiencies in the stories their generators create [14][15].

A common thread between both academic and industry problems is that tools for evaluating subjective aspects of games such as their narratives are lacking. We propose with this research that narrative concepts, particularly structuralism, can be used to model game stories. Our primary research question is whether this structural model can adequately describe game narrative and distinguish between or identify "good" and "bad" stories, based on differences in structural properties. To answer this, we developed several sub-questions: 
1. What components of the narrative macrostructures are present, and are the overall macrostructures we have chosen well suited to game stories?

2. Can the source of a story event, as either internal or external, be easily determined, and is it a useful metric for modelling story events?

3. Does this model accurately provide a picture of how events are sequenced together in a typical game story?

4. What is the relationship between the source of story events (as either character-driven or plot-driven) and their ordering, and the quality or success of the story?

5. Are there other factors which affect this relationship, such as the importance of a story event as either a Kernel or Satellite?

\subsection{Significance}

A model for game narrative analysis would have many applications, both academic and industry. In general, game story writers could benefit from a structural model, as either a starting point for their stories or a method to evaluate a written story. Larger studios with high budget and high-risk games may also benefit from a tool which could allow them to better evaluate their in-development projects and potentially reduce the risk of commercial failure. Evaluation in this way may also reduce the need for costly user testing sessions, which could lead to cost savings against the game's budget.

In academia, we see procedural generation of narrative as a field which could benefit from this model. Evaluation of content is one of the key challenges when designing content generators, particularly for subjective content such as narrative [11]. Our model could be used 
as a metric to evaluate generated stories against. The model could also be used in the generation process, to better inform content selection.

Procedural generation of quality narrative can increase the replayability of a game [16]. If players enjoy the mechanics or design of a game, having unique narrative campaigns could keep them playing longer than if they had to repeat the same campaign.

Better story quality might lead to industry adoption of procedural generation techniques in commercial games. This has benefits for two sectors of game development: small teams, and rapid content release games. Smaller teams that have fewer development resources available could benefit from offloading the narrative to a generator, allowing them to focus their resources on other game features such as gameplay or asset production. Additionally, we see applications within games that are designed for continual content release to their player base, such as Massively Multiplayer Online games. Developer production of content, even in large teams, often cannot keep pace with player consumption. Procedural generation of narrative content could again, increase the output of development by allowing developers to allocate resources elsewhere.

\subsection{Contributions}

To answer the above research questions, we developed:

- A method for data collection and presentation for game stories

- A visualizer to present this data

- A proposed model for game stories based on analysis done on our collected data 
The major contribution of this research is the creation of a structural model for game stories. The model is event-based and contains information about the types of events within a story, and their ordering.

To create this model, we also developed several tools with which to analyse game stories. We designed a method for discretizing game stories into their individual events and defined a list of properties that could be categorized for each event. We defined a new property for story events, where an event could be either "character-driven" or "plot-driven". This categorization was designed to cover the narrative concept of internal and external character goals. We also used the structuralist properties of "Kernel" and "Satellite" to classify the importance of an event within the story.

We developed a story visualization tool which allowed us to graphically view different properties of our collected story data. This visualizer allowed us to both draw initial conclusions and refine our research questions.

Both qualitative and quantitative analysis was done on our collected data. Statistical analysis was done when possible on quantitative data. We were able to confirm the details of our model by accepting or rejecting each of the categories we studied as useful. Our results also suggest that the implementation of internal and external character goals has a strong relationship with the quality of the game story. Based on these results, we were able to suggest guidelines for the overall event structure of a narrative. This structure is presented in terms of narrative macrostructures, character-driven/plot-driven, and kernel/satellite events. 


\subsection{Thesis Structure}

Chapter 2 will give an overview of relevant information and research. Narrative, storytelling, and the components of these concepts will be outlined, as well as their relationship and applicability to the medium of video games. We will also overview Procedural Generation of Content (PCG) for Video Games, and study the various approaches to narrative generation that have already been explored.

We will discuss our research questions and our methodology in Chapter 3. Chapter 4 contains the results of our study. In Chapter 5, we will discuss our findings, and Chapter 6 contains our conclusions and a discussion of future potential work.

The remainder of the document contains our appendices, which further elaborate our data collection and analysis methods. 


\section{Related Work}

In this chapter, we give a review of the relevant concepts to our work. We begin with an overview of narrative and its relationship to video games. We then discuss various methods for interactive storytelling. We cap our discussion of narrative with an overview of structuralism. We next move on to a discussion of procedural generation and its benefits. We finish the chapter with a review of the different methods for narrative generation, identifying their weaknesses, which we hope to address with this research.

\subsection{Narrative and Storytelling}

Storytelling is a quintessentially human activity. Since the earliest civilizations, we have lived our lives surrounded by stories [1], we have communicated through them [2][17], and they have inspired us to reach, create, and further ourselves [18]. Despite being a popular and common activity, storytelling remains a complex process. The study of narrative is an equally complex field, full of many theories, arguments, and debates. While any person can describe a sequence of events, these do not always make for interesting or entertaining stories. In this section, we will discuss narratives from the scholarly perspective, and focus on the components of a "good" story.

A narrative is made up of two parts. The first is the plot: the actual sequence of events [1], which is termed the story [19]. A story event is one action within the overall sequence. Any story could, therefore, be broken down into a list of these story events. The term "story" is often used to refer to "narrative", however, here we will distinguish the two. The story is one component of narrative. The second component of narrative is the manner in which that story 
is told, or the discourse [19]. The order that the events are told may differ from the order they occur in the story. Information may be withheld, or events may be revealed to the reader out of temporal order through flashbacks or foreshadowing, alongside other storytelling techniques. Altering the discourse of the sequence of events creates a narrative. Both the story and the discourse of narrative have been heavily analysed.

Good narratives can generally be boiled down to the same basic outline: a protagonist who has a goal, and who overcomes a series of difficult challenges in order to achieve it [1][2][20][5][4][17]. The "goal" may not take the form of an outright objective. Propp has termed it as a "lack" or misfortune[5], that is to say, a deficiency in a character's life. It may also appear as a disequilibrium or perturbation in the world around the character.

Stories are about people [2][3]. The actual characters may not be human, but they always should represent people. Sometimes, characters are added purely to serve a function of the plot. This should be avoided; characters should be considered entities of their own, instead of as the means to perform a plot function [1][19]. The world around the characters and the things in it may feature prominently in the narrative, but the focus should always be on the people, not the things [17]. Going one step deeper, the story is about the choices made by the characters and the changes they experience as a result of their journey [1][2]. These internal experiences are the true driving force of the story [2]. In strong stories, external problems and challenges thrown at the characters are related to the character's internal issues. Characters move towards overcoming their internal problems through the completion of external events [2]. In Star Wars [21], for example, Luke Skywalker's external goal of joining the Jedi and 
stopping the Empire is rooted in his internal issues surrounding the identity of his father, and his own identity. In The Matrix [22], Neo's internal goal of understanding the truth about himself and the world around him is achieved through the external acts of learning to survive in the real world and fighting off machine attacks both in and out of the Matrix in order to save his friends. In Neo's case, he becomes The One, an external manifestation of the resolution of his internal desires.

Narratives should contain some form of conflict [17]. Suspense and surprise [5][9], and curiosity are other factors which can affect user enjoyment of a story [23]. Surprise, for example, is described as the omission of key information earlier in the discourse. By placing the information later in the discourse, the reader is surprised as they make sense of the new information in the context of its omission [23]. Suspense occurs when an event can lead to "significant consequences" to a character, which can be positive or negative [23]. If a perceived solution to a problem is removed, this can also heighten suspense in readers [24]. Curiosity is the deliberate omission of an event, with the reader being aware that information is missing. Entertainment is a key component of a story [17], and some theorists even argue that narratives without an entertainment purpose are not stories at all [23].

\subsection{Narrative in Games}

Video games as a medium contain far more than just the narrative. Narrative is not even required in order to have a good game [1]. In academia, the study of video games has at times been a topic of great debate between narratologists and ludologists [25]. Ludology, a term coined by Gonzalo Frasca, studies games purely on their mechanics and "play activities" [26]. 
Ludologists reject the application of literary theories to games, arguing that doing so causes us to lose sight of what sets games apart from other media such as film or print. Narratologists, on the other hand, see video games as a new medium for storytelling, as discussed above. In recent years, the debate has mostly died down, with a compromise being reached from both sides: Narrative is not a requirement for a game, however, games can be used as a medium to tell stories [1].

Narrative can provide strong framing for player actions within the game and can offer players motivation to continue playing [18][9]. This occurs both in the sense of providing the player with a desire to know "what comes next" [9], but also providing emotional context to the game's tasks. As Salen and Zimmerman write,

"It does not make much narrative sense to knock down rows of colored blocks if the behavior of those blocks has no connection to your presence in the game world. Once you identify those colored blocks as a force field designed by the forces of evil to stop your advance through the universe, you are much more motivated to enter into a conflict with them. Your action becomes meaningful within the narrative frame of the game" [9] Games as a medium also provide many other avenues through which they can tell the story. This includes cutscenes, dialogue, level design, player controls, and the gameplay mechanics themselves. Games can provide a strong medium for storytelling, as gameplay is typically built on a recursive structure, much like narrative conflict. Such a structure involves challenges and rewards become increasingly large as the game/story progresses, building to a climax [27]. The 
interactive component of video games has allowed them to emerge as a medium for telling interactive stories.

\subsection{Interactive Stories}

Chris Crawford defines interactivity as "a cyclic process between two or more active agents in which each agent alternately listens, thinks, and speaks" [17]. Traditional written forms of storytelling are non-interactive, in that the reader or consumer has no ability to affect the narrative. When the user is given some agency over the events and outcome of the narrative, it becomes "Interactive Fiction". As computers have become more widespread, interactive fiction has often been seen as exclusive to that domain [28]. However, interaction is present in many forms of "traditional" storytelling. The I Ching is a form of interactive storytelling which was written thousands of years ago, where user-generated "hexagrams" are used to decide which passages are read [29]. Choose Your Own Adventure books are a more recent example. The capability for user interaction affecting the story was first referred to as "Interactive Fiction" by Niesz and Holland [28].

Narratologists agree that the fundamental concepts behind narrative and storytelling are independent of medium [1][30][19][3][31]. Janet Murray's pioneering book, "Hamlet on the Holodeck" sought to explore how digital media and the interaction they provide could lead to better storytelling experiences for users. Murray disagrees with the term "suspension of disbelief", and instead proposes that story listening is not a passive activity. Listeners take an active process in creating their belief"[28][30]. Interestingly, Crawford disagrees with this idea, saying that the amount of thought put in by the reader will never affect the narrative, so it 
cannot be an active process [17]. Murray's view, by contrast, is that in traditional narratives we participate, but we do not have agency [30]. She, therefore, proposes that interaction in digital media can be used to reinforce and take advantage of the reader's active process of "listening" to the story, ultimately strengthening immersion.

\subsubsection{Types of Stories}

Most non-interactive forms of storytelling are termed linear, that is to say, the sequence of events will occur in the same linear order every time the narrative is read.

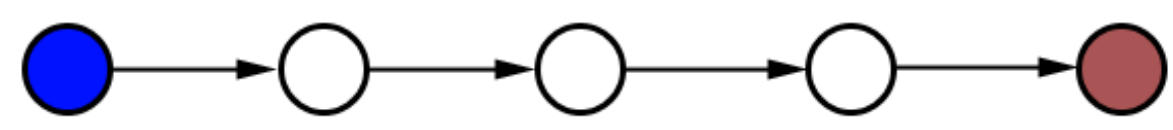

Figure 1. Graphical representation of a linear story, with each node representing a story event.

Niesz and Holland's description of interactive narrative is that of a tree-shaped branching narrative, where player choices lead to a series of pre-authored outcomes [28]. Branching narratives have the disadvantage of requiring exponential work on the part of the writer, while the resultant story is still a fixed number of steps as it would have been in a linear presentation [17]. To limit exponential ending growth with every choice, "fold-back" structures can be used, where different choice paths converge to the same ending. However, this reduces the impact of user choice and can make users feel that their choices do not truly affect the story.

Hypertext narratives are another form of non-linear storytelling. Hypertext narratives are similar to branching narratives, in that story segments are written, and user choices will link them between these segments [32][1]. However, story segments are more interwoven, with 
more possibilities for moving between narrative segments. Segments can also be visited multiple times, and loops are possible, which is not the case with branching narrative. Figure 2 illustrates branching and hypertext structures. The "Choose your Own Adventure" series of books use a hypertext structure.
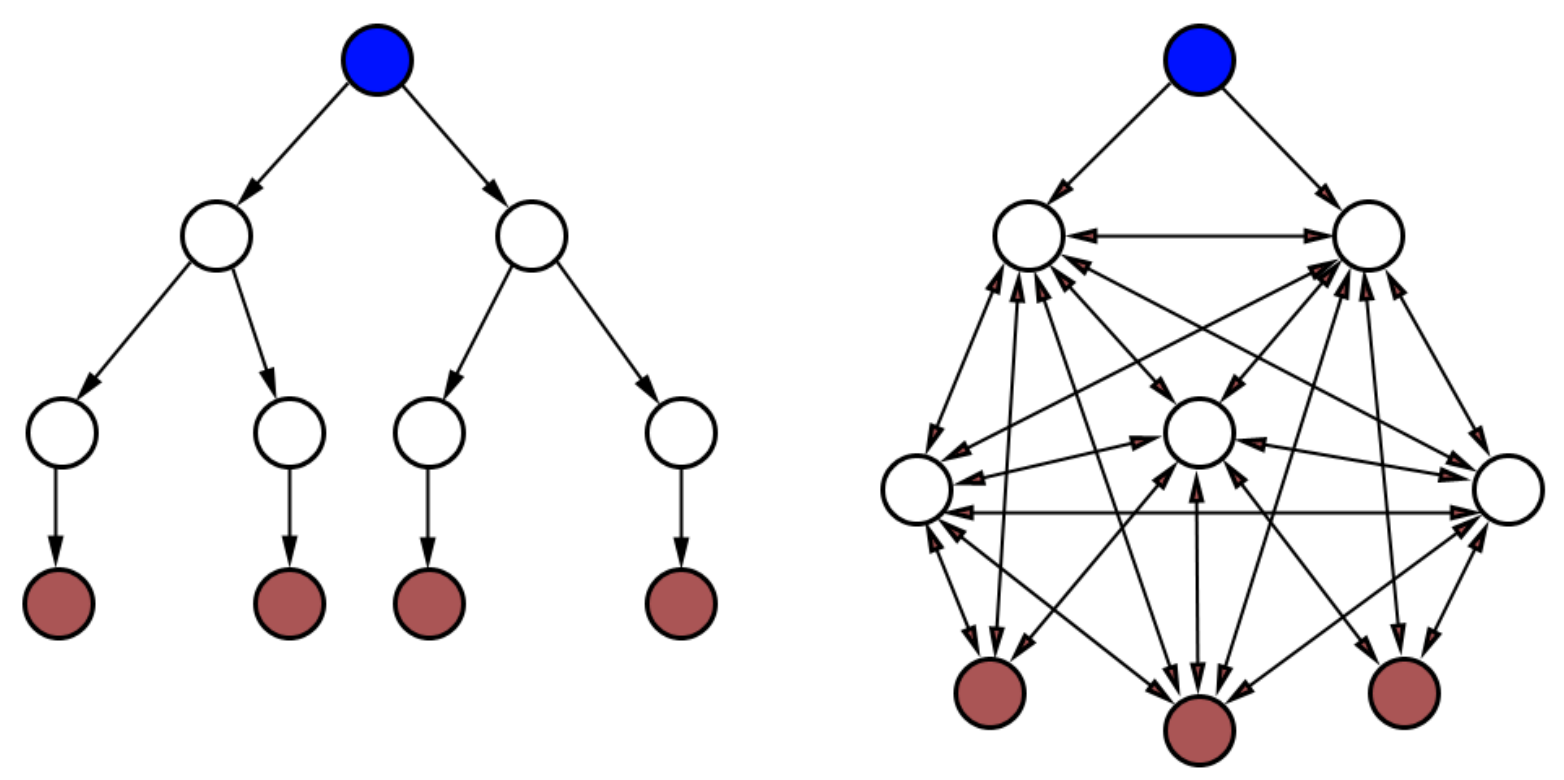

Figure 2. Branching and Hypertext sample structures. Start nodes are coloured blue, while end nodes are coloured red.

\subsubsection{Player versus character}

It is important to distinguish here between the user, or in games the "player", and the character within the story. In a traditional linear story, Montfort describes this relationship as the user playing the game and directing the character, but not playing the character in the dramatic sense [33]. The author, not the player, decides the character's actions and reactions, and the player is responsible for "steering" the character through the narrative [33]. Even in interactive stories, it is important to view the player as a separate entity from the character they are operating. It is clear that, at least in games, there are two types of interactivity 
possible. A game is interactive by virtue of the medium, even if the player cannot affect the narrative [18]. The game story can also be interactive, or "player-driven" when the player themselves is able to exert control over the outcome of the story [1][18].

\subsubsection{Embedded and Emergent Spectrum}

Interactivity in storytelling falls along a spectrum, from non-existent to fully user-driven narratives [18], termed "embedded" and "emergent" narratives respectively. Embedded narrative is the traditional storytelling approach: the story is pre-written, and "embedded" into the story. Player interaction cannot change the story, and the player is much like the reader of a book [9]. Emergent narrative, on the other hand, is the narrative experience created in the player's mind based on their experiences in the game. Games such as Minecraft or the Sims contain little to no embedded story. Players create and act out narratives based on their own interests and goals within the framework of the game's capabilities. Most games combine different amounts of embedded and emergent narratives. However, even games with embedded stories will produce different emergent experiences in their players [9].

The spectrum between embedded to emergent can take many forms. A purely embedded story is a strictly linear sequence of events, experienced by the player, with no opportunity for them to influence it. Branching narratives can give the player choice over a series of pre-authored story paths, allowing them to influence which story they ultimately experience [18]. Open-ended stories take the concept of branching even further and provide players more options for approaching the game content. These tend to be the more "open- 
world" style games, where players are free to approach and complete content in whatever order and method they choose [18].

\subsubsection{Challenges}

Successful player-driven narratives are rare and challenging to create. Narrative audiences will generally act to reduce tension and avoid conflict [1] and may lack personal qualities such as adequate creativity to create a compelling story [34]. As we have discussed above, factors such as suspense and surprise are critical to good narrative [1][23], and so players are less likely to make story choices which would lead to an interesting narrative. Narrative in traditional media has often actively tried to limit or remove audience participation, in order to avoid this problem and sustain narrative immersion [30]. Explicit player control over a narrative is also not always a desired trait from the player perspective. As Glassner writes, "Audiences do not want to do the author's work" [1].

\subsection{Narrative Structuralism}

Narratives contain a story, made up of events, and a discourse, or the ordering of those events and how they are told to the reader. While events can be told in the order they happened, reordering of the discourse can elicit more emotions or satisfaction in the reader during their experience of the story [23]. Narrative structuralists are concerned with the analysis of stories from this perspective and examine story events both individually and within the context of their overall placement within the discourse.

Vladimir Propp's dissection of the Russian Fairytale in 1928 was a pioneering structuralist work. Propp broke down these narratives into 31 "functions", which he found to be common 
between a large number of stories [5]. Each of these functions was given an accompanying symbol, and he was able to structuralize stories by listing their events in terms of their function symbols. With regards to the discourse of these functions, Propp indicated that in his study of tales he found no requirements for specific ordering. Some functions might be repeated multiple times in a tale, or occur "out of order", while others may not be present at all [5].

Joseph Campbell identified the popular macrostructure "The Hero's Journey" in his book "The Hero with a Thousand Faces" [4]. This structure divides the typical adventure plot into 17 stages. Christopher Vogler adapted and simplified Campbell's structure into a twelve-step version known now as the Writer's Journey. Vogler summarizes a story following this simplified structure as follows:

"The hero's journey, once more: The hero is introduced in his ORDINARY WORLD where he receives the CALL TO ADVENTURE. He is RELUCTANT at first to CROSS THE FIRST THRESHOLD where he eventually encounters TESTS, ALLIES and ENEMIES. He reaches the INNERMOST CAVE where he endures the SUPREME ORDEAL. He SEIZES THE SWORD or the treasure and is pursued on the ROAD BACK to his world. He is RESURRECTED and transformed by his experience. He RETURNS to his ordinary world with a treasure, boon, or ELIXIR to benefit his world." [6]

The Hero's and Writer's journeys propose a more rigid discourse structure compared to Propp's work, where all the functions play out in chronological list order. Propp and Campbell's analyses contain many shared functions and an overall similarity in structure, although with 31 functions Propp's definition allows for more detail in the categorization of story events. Not 
every event in a story must correspond directly with a function from one of these structures, but the overall flow of an adventure plot generally adheres to them. More generally, these structures all adhere to the general description of a story we described earlier: "a protagonist who has a goal, and who overcomes a series of difficult challenges in order to achieve it $[1][2][20][5][4][17] "$. It is interesting to note that Vogler interpreted the hero's journey as actually containing two major ordeals. While the original problem is resolved with the first ordeal, this usually gives rise to a second challenge that must be overcome before the overall quest is truly finished. Vogler's interpretation agrees with Propp, who proposed that most tales contain two "moves" of action. A list of the various functions and events from each structure and a comparison between these macrostructures can be found in Appendices 1 and 2 .

Barthes structuralized narrative on three levels: "functions", "actions", and "narration" [3]. Barthes' function level echoes Propp's definition, that is, the individual story events. His "narration" level corresponds to the discourse of the story. Actions lie between the two. A function derives its meaning from its place within a line of action, and the action is meaningful only through how it is communicated through the discourse [3].

Chatman identifies two types of story events: kernels, and satellites [19]. Barthes identified the same hierarchy within his functions, using the terminology of cardinal functions and catalyses [3]. Chatman's terminology is used here as it is the terminology featured in this work. Kernels are story events which are required to understand the logic of the story. They cannot be omitted without altering the plot. Satellites are the opposite. They add to the texture of the story, but if they were to be omitted, the story would remain essentially unchanged, and could 
still be understood by the reader [19][3]. Barthes' Actions level is concerned with characters. Actions as a structural level are a more broad concept than the lowercase "a" actions that make up the functional layer. He believed characters should be defined by what they do, rather than their traits [3].

Games tend to gravitate towards action and adventure stories. It is much rarer to see romances or sitcoms, though they do exist [18]. Narrative macrostructures that center on adventure-type plots, such as the Hero's Journey, and Propp's morphology, are therefore excellent choices with which to analyse game narratives [18][19]. However, the other structural elements we have discussed here are not often discussed in-game narrative research.

\subsection{Procedural Content Generation Overview}

Procedural Content Generation (PCG) is defined as "the algorithmical creation of game content with limited or indirect user input" [35]. PCG is an active field of research and sees widespread success in commercial titles. In games "content" can be nearly anything. Procedural generation has been used to create objects [12], animations [36], dungeons [13], quests [10], narratives [37], and game mechanics [11] among others. Games such as Minecraft [38] and No Man's Sky [39] have drawn attention with their infinite procedural worlds. Procedural generation has also seen widespread commercial success through development tools such as Speedtree [12], which creates trees and vegetation without the need for hand modelling.

PCG has many advantages when applied to the medium of video games, which contribute to its continued popularity in both commercial and academic circles. Procedural Generation allows for instant customization and tailoring of a game to each player, which has been shown 
to increase player enjoyment [40]. With its capability to produce constantly novel content, procedural generation has been proposed as a method to both extend or create theoretically infinite replayability in games [41]. New and unique genres of play may be possible with procedural generation techniques [41]. As technological capabilities of games increases, development team sizes have substantially increased [7]. Procedural Generation can be used as a tool to augment the capabilities of existing human designers and developers, helping to keep costs and team sizes manageable without compromising on quality [11]. No Man's Sky, for example, was a major AAA release, with a development staff of only 15 [39].

This is not to say that PCG is a panacea for game development. There are many challenges facing the field, both academically and in the industry. Evaluation of generated content remains a struggle. Given that generators are designed to create novel and varied content, it is difficult to guarantee that every possible generation will meet requirements [11]. Game creators also have different desires from content generation than players may have, and evaluation can be done from either perspective.

Generating content which is varied, and yet novel, is a challenge. Too little variation and generated content can be boring. Too much stochasticity and the content may not feel as though it belongs in the game [11]. Along similar lines, balancing generator freedom with the controllability expected from a game designer is another difficulty.

\subsubsection{Narrative Generation}

Generation of narrative is an active field of PCG research. Narrative generation shares the same advantages of PCG as a whole: namely the ability to add interaction and 
customizability to a game, which increases player immersion [40]. It can augment the abilities of human writers, allowing them to create more story content in less time. This can lead to games with campaigns that never end, or allow content patches to be more frequently released.

Narrative generation shares the overall challenges faced by procedural generation techniques, with additional challenges of its own. Evaluation is uniquely difficult in narrative generation, due to the subjective nature of stories. Researchers generally make use of human studies to combat this [10][42], with techniques such a Turing-style test where users are asked to guess which story was computer authored [43]. Significant work has gone into developing new ways of evaluating generated narratives, against various metrics such as novelty [44], uniqueness, or surprise [45]. In general, evaluation of generated narratives remains an outstanding area of research.

A second challenge when looking at narrative generation for games is the two-fold nature of stories. Both the story events and the discourse must be generated to a high degree of quality for any narrative generation system to be considered a success. As we will discuss below, typically generation systems choose to focus on either one or the other. It is rare for systems to attempt both. While this can still be interesting from a research perspective, a successful commercial generator would require both components.

A particular debate within narrative generation is why it should be generated at all. One of procedural content generation's big advantages is its allowance for dynamic, or endless content. However, with narrative, we have shown that this is not necessarily desirable. While 
procedural generation can further the medium of games through new gameplay genres, mechanics, and so on, it is very unlikely that the process of storytelling will see much innovation from PCG research. Endless stories, while possible, contradict narrative theories that suggest all narratives must have a clear endpoint [4][5]. Additionally, as we have shown, player agency over the story has a high likelihood to negatively alter the story experience [1][30].

Despite these setbacks, generation of narrative has many applications and implications. Generation of narrative could assist with creating serialized or episodic content. Episodic stories have seen great success in television shows, and have in recent years become more popular in games with titles such as Life is Strange, or games produced by Telltale Games. MMORPG games tend to feature relatively quick content cycles, and often player desire for new content outstrips development pace [16]. A commercially viable narrative generator, especially one with a robust discourse or quest generation component, seems well suited to address this issue.

Outside of practical applications, research into narrative generation presents an opportunity to learn more about ourselves as people. Storytelling is a fundamentally complex process, which has been studied for millennia [1]. If we reach the stage where we can automate the process of storytelling, then we must on some level finally understand both storytelling, and how it affects the people who both compose and consume stories. With every step forward in this field, we come one step closer to understanding this complex human activity.

\subsection{Approaches to Narrative Generation}


Procedural narrative generation has been widely explored from many angles. In this discussion, we categorize systems based on their approach to narrative generation, building off the themes discussed previously. These categories are emergent and player-driven narratives, character-driven simulations, author simulation, and discourse generation. While every approach has its merits, there remain weaknesses with all the approaches. We feel that these weaknesses are a result of a lack of application of structuralist concepts to the narrative generators.

\subsubsection{Emergent Narratives / Player-Driven}

Chavin et al. propose an architecture for emergent narrative generation which is built off of what they term "Narrative Processes" and an "Interpretation Engine". Narrative Processes are player-activated scripts which modify the game world and story in real time. The Interpretation Engine is the system responsible for maintaining knowledge of the story world, which the Narrative Processes can then access in order to make more meaningful changes to the story. This framework for narrative generation considers the player and the character to be one and the same [34].

PaSSAGE (Player-Specific Stories via Automatically Generated Events), takes what they term to be a "player-modelling" approach. As the game is played, PaSSAGE creates a player model, which is then used to draw from a database of story branches and "encounters" [46]. While the player is not conscious of their effect on the story, the resultant narrative is completely player-driven. 
This approach to generation often creates low-quality narrative experiences, due to explicit player-control over the story [1][23][34].

\subsubsection{Character-Driven}

Character-driven systems generally operate by creating models of characters and allowing them to autonomously interact with one another in order to generate a narrative. Meehan's TALE-SPIN is one of the earliest attempts at procedural story generation [37]. TALESPIN was developed off of Alan Perlis' vision of what he termed "the metanovel", a computer program capable of telling stories in a way only possible through that medium. TALE-SPIN tries to model the knowledge required to tell a story, both the world and characters with motives, emotions, and relationships. It tells a story by creating "a simulation of rational behaviour by the characters in the model" [37]. Meehan found that while the system was able to generate coherent stories, most of them were not very interesting.

Divine Intervention Operator Service (DIOS), generates stories from agent-based social simulations. The system was designed to answer the question of whether this type of simulation model is capable of creating interesting narratives. The results of the DIOS study showed that it is possible for such a system to create interesting narratives and that the frequency with which they happen could be somewhat controlled [47]. However, an interesting narrative is not guaranteed from a free running character simulation. Given that a key factor of a generator is its ability to guarantee certain properties of its output [11], this poses a problem for purely character-driven generators. 


\subsubsection{Author Simulation}

Another approach to narrative generation arose from the weaknesses of characterdriven systems. These systems attempt to assert more control over the narrative by modelling an author. Dehn's AUTHOR, for example, models an author of the story, an entity who has its own memory, knowledge, and goals that are independent of the characters within the story [48]. Dehn discusses the importance of, and the difference between character goals and author goals. However, AUTHOR implements only Author goals, in contrast to TALE-SPIN, which implements only character goals.

Turner's MINSTREL is another generation system which attempts to model the "cognitive processes of storytelling and creativity" [15]. Storytelling was approached as fundamentally a problem-solving exercise. MINSTREL attempts to fill four authorial goals: to make the story interesting, understandable, artistic, and creative. MINSTREL was limited to the domain of King Arthur and the Knights of the Round Table and was able to create text-based stories which were perceived by users to have been written by children in the 10-15 year age group [15]. Turner was struck by the extreme complexity and knowledge required for the system to be able to generate even the simplest of stories.

Tension and suspense are key to a good narrative, as we have discussed previously [1][23]. Suspenser, a narrative generation system, was built around the idea of creating suspense within its narratives. Suspenser follows Bernardo and Gerrig's model [24], which relates reader suspense to the number of possible solutions they can imagine to a character's problem. This is another type of author modelling approach. 
Purely author-driven approaches lack the character internal goals that Cron has identified as part of a successful story. If authorial events are not related to a strong set of character goals, the overall story quality suffers [2].

\subsubsection{Hybrid approaches}

The Virtual Storyteller is an agent-based system consisting of both character agents and a "director" agent. Character agents create plans towards achieving their goals. The director determines whether this action fits within the bounds of the plot and whether it will likely lead to an interesting story. The director cannot force a character to take an action but can affect the story world to encourage character action. While The Virtual Storyteller contained no player interaction, the authors expressed interest in allowing users to perform some of the director agent's tasks [49].

Fabulis is another system which attempts to combine "character-believability" with "plot-coherence" [14]. Fabulis generates the story from an open world: the algorithm searches the set of characters within the world for ones whose personalities and goals fit what the story needs. Characters are not human-authored. An authorial model is used to keep plot-coherence by constraining the character simulations within the bounds of the story, in a very similar fashion to The Virtual Storyteller.

Systems such as The Virtual Storyteller and Fabulis avoid the shortcomings of both character-driven and authorial simulation by combining them in this hybrid approach. However, the story plans generated by these systems fail to take into account the narratological theories and structures described earlier in this chapter. These concepts have been shown to be 
necessary components of a good story. Fabulis, in particular, identifies the lack of a structural model of narrative in their system as a weakness [14]. Additionally, while Fabulis contains both a story content and a discourse component, it considers both aspects to be separate, rather than part of the overall story [14].

\subsubsection{Discourse and Quest Generation}

As we have seen so far, the bulk of generators are focused on creating the content of the story, that is, the story events. However, the discourse of a story is equally important to narrative. In generation research, we have found the terms "quests" and "narrative" to be at times used interchangeably. Quests are a form of the narrative's discourse; they are a way to integrate the narrative with the game's mechanics and objectives [11][50]. Quests themselves are not the narrative, however, they create space for the narrative to occur [25]. Systems such as ReGEN attempt to compare their generated narratives to those of other systems. However, ReGEN's narratives are restricted by the limitations of its quest generator. The true capabilities of their narrative generator are, therefore, not accurately showcased [10]. Any story generator will need a robust companion quest generator in order to create a fully realized narrative generation system.

Prevoyant [45] does not generate stories but modifies the discourse of an input story through flashback and flashforward devices in order to elicit surprise in its reader. Their study raised the question of how much complexity in temporal discourse rearrangement was ideal for readers: too little and readers were bored, too much and they became confused [45]. Given 
that both story events and discourse are key components of any story, this poses a problem with regards to story generators.

\subsection{Gap Analysis}

Purely character-driven systems are unlikely to generate interesting narratives [37][14]. Though it is possible for interesting narratives to arise, a "good" generator should be predictable and consistent [11], and character-driven systems are neither. On the other hand, generators which follow a pure authorial simulation produce stories which are not peoplecentric or rooted in strong relatable character goals [2][14].

Frequently, narrative generators focus on either the story events, or the discourse, but not typically both. Fabulis [14], while addressing both the story and the discourse, did not do so from a structural perspective. It considered the story and the discourse to be separate entities when, in reality, both the story events and their ordering make up the narrative as a whole. Generators which focus solely on story content tend to produce stories where events are communicated in a linear and chronological fashion. Discourse-focused systems such as Prevoyant were unable to draw clear conclusions about how much rearrangement of story events was ideal from a viewer perspective [45].

All the generators we reviewed approached narrative generation from a content perspective. We found no generators which made use of narrative structure to inform this content. That is to say, we saw no generation from the perspective of a narrative containing both a sequence of story events, each with individual properties, which are ordered to create a 
discourse. Structuralism provides a large amount of additional data about the story, which we believe could aid in the selection of which content to generate and in what form it should take. This type of story model is well suited to an algorithmic approach to narrative generation. From a generation perspective, the ability to view a story as a sequence of events, each with properties and functions related to it can simplify the process of narrative generation. Additionally, we believe that this type of story model could assist in the controllability factor of a generator. By having an established model to compare to, stories created by a generator could be better evaluated to ensure successful outputs.

We propose with this research to define an event-based model of game story structure. Such a model will include information about the types and orderings of events within game stories. We propose to define this model through a study of existing game stories. By compiling a dataset of structuralized game stories, we will be able to draw wider conclusions about the structure of game stories as a whole, both in terms of story event properties and the resultant discourse. 


\section{Research Approach}

The goal of this work is to create an event-based model of game story structure. We propose to create such a model by studying existing game stories through the structuralist lens of a purposefully ordered sequence of events. We propose that the model will consist of a sequence of events, which may or may not be presented to the player in the same order in which they occurred. We predict that the model will be based on strong goal-oriented characters that both drive events and react to external events. We propose the model include existing structuralist concepts such as Kernels and Satellites and narrative macrostructures such as The Hero's Journey. Additionally, we propose a new categorization of story events which is based on the concept of internal and external character actions. These categorizations will allow conclusions to be drawn both at the individual story event level and at the discourse level of the narrative.

\subsection{Character-Driven and Plot-Driven Events}

A story is about a protagonist who has an internal goal. The protagonist overcomes a series of external goals and challenges in the hopes of achieving their internal goal [2]. We propose a categorization for story events based on this internal/external binary, using the terms "character-driven", and "plot-driven" to refer to internal and externally sourcing events respectively. Essentially, a story revolves around character-driven events performed by the characters as a reaction to plot-driven events, with both types of event driving the narrative towards the character achieving their internal and external goals [1][2]. 


\subsubsection{Character-Driven Events}

We consider characters within a narrative to be entities themselves, with personalities and goals that they are disposed to working towards [1]. We consider a story event which occurs as a result of a conscious decision by a character within the story to be "characterdriven". The key here is that there are multiple courses of action available to the character, and it is their choice which determines how the story proceeds.

Some events can be carried out by a character, or involve a character without being character-driven. The event must contain a character choice or intent in order to be considered character-driven.

\subsubsection{Plot-Driven Events}

As we have discussed above, it is the author's job to create external issues for the characters to overcome. These author-sourced events are what we term "plot-driven". These are events which happen to characters, as opposed to the characters driving the event.

Plot-driven events can be events that occur regardless of character actions or events which would have occurred regardless of which character(s) are involved. Even if a character performs the action, if they have no choice in the matter, the event cannot truly be characterdriven. Using Cron's terminology, these are "external" events, which source from outside the character [2]. 


\subsubsection{Examples}

\subsubsection{Character-Driven}

Metal Gear Solid 3: Volgin orders Ocelot to shoot Snake, but he refuses, citing a promise to The Boss.

Ocelot makes the choice to refuse his orders because of a personal promise with another character.

Final Fantasy X: At the temple, Wakka is worried that the Summoner has not returned yet. Tidus rushes in to help despite it being forbidden for non-summoners or guardians.

Tidus chooses to disregard the rules about his ability to enter the temple because Waka's worry has awakened his desire to help others.

The Last of Us: Ellie and Joel travel to meet Bill, who owes Joel a favour. Bill agrees to fix up a car if Joel and Ellie agree to help gather the parts.

Bill agrees to help Joel and Ellie but only under a condition. Because the character of Bill actually has agency here, his decision to help if his demands are met makes this a characterdriven event.

\subsubsection{Plot-Driven}

The Legend of Zelda: Ocarina of Time: Link has aged 7 years, as he was too young to be the Hero of Time

This event happens TO Link, with no input from him or any other characters. 
Uncharted: Drake's Fortune: Elena triggers a trap, locking Sully away from Drake and Elena.

This is a plot-driven event because Elena was not aware of the trap, and did not intentionally trigger it.

\section{Destiny: They use the Eye to gain access to the chamber of the Black Garden's Heart.}

This is a plot-driven event because it is merely the protagonist carrying out the external goal assigned to them. No internal character goal or choice caused this event to occur.

\subsection{Research Question}

Our overall question is whether a game story can be modelled structurally in a useful way. That is, can we model a successful story and distinguish it from an unsuccessful one? This overall question is comprised of many parts:

1. What components of the narrative macrostructures are present, and are the overall macrostructures we have chosen well suited to game stories?

2. Can the source of a story event, as either character-driven or plot-driven, be easily determined, and is it a useful metric for modelling story events?

3. Does this model accurately provide a picture of how events are sequenced together in a typical game story?

4. What is the relationship between the source of story events (as either character-driven or plot-driven) and their ordering, and the quality or success of the story?

5. Are there other factors which affect this relationship, such as the importance of a story event as either a Kernel or Satellite? 
By performing our analysis, we hope to confirm that these narrative theories, categorizations and macrostructures are applicable to the medium of games and that a clear model of a successful game story can be derived. Such a model would be beneficial to inform a future generator.

\subsection{Research Method}

This section will outline the process by which we collected our data. Afterwards, we will describe the analysis tools we developed and our overall methodology for studying our data.

\subsubsection{Data Collection}

In order to determine structural data about game stories, we analysed 20 existing game stories. Games were selected across multiple genres. In order to draw conclusions about the analysed stories, it was necessary for us to define some metric for quality. Since the perception of a narrative is subjective, it is difficult to find an objective rating for quality. We decided to use the aggregate critical score of each game, drawn from a set of popular review sites (IGN, GameSpot, GamesRadar, RPG Fan, Electronic Gaming Monthly, and Gamelnformer). Not every game was present from every reviewer, but this allowed us to get an aggregate score of at least 3 reviews per game. While we are aware that game reviews encompass far more than just the story quality, games were selected only where the story featured prominently in the game reviews.

In addition to a prominent story, we selected only embedded narrative games. Our selected games, if not fully linear, involved simple branching narratives, where each ending was fully embedded, and where there were infrequent choices/branches. No hypertext stories were 
chosen to study. Games were selected from a variety of genres, although some genres feature more prominently due to them being better suited for complex narratives. Role-playing and adventure games were most common, although first-person shooters were also analysed. As discussed above, the score of the games was used as a quality metric. We attempted to analyse both high and lower scoring games, to see if inferences about story structure could be made by viewing the differences between higher and lower scoring game structures.

When at all possible, games were personally played through to collect data. For games that were difficult to locate, online playthrough videos were used to experience the story. Playthroughs were chosen without any creator commentary or dialogue to influence the perception of the game story.

\subsubsection{Data Analysis}

For analysis, we broke down the game stories into discrete events. The "plot point" as described by Glassner [1] is the bar for a story event in our data. This is described as "when something happens to change or advance the story" [1]. We categorized each event according to several classifications, which are described below. Table 1 gives a sample table illustrating our data. 
Table 1. Sample Data Sheet for "The Legend of Zelda: Ocarina of Time". This sheet does not show the entire game, just illustrates a few data point examples.

\begin{tabular}{|c|c|c|c|c|c|c|c|c|}
\hline EventType & Importance & Arc & EventDescription & $\begin{array}{l}\text { Characters } \\
\text { Involved }\end{array}$ & $\begin{array}{l}\text { Character } \\
\text { Driver }\end{array}$ & $\begin{array}{l}\text { Propp's } \\
\text { Function }\end{array}$ & $\begin{array}{l}\text { Hero's } \\
\text { Journey }\end{array}$ & $\begin{array}{l}\text { Writer's } \\
\text { Journey }\end{array}$ \\
\hline Plot-Driven & Satellite & Main & Link dreams of a strange evil man. & Link & & & & \\
\hline $\begin{array}{l}\text { Character- } \\
\text { Driven }\end{array}$ & Kernel & Chapter & $\begin{array}{l}\text { The Deku tree sends Navi to fetch } \\
\text { Link, whom he believes can defeat } \\
\text { the evil he feels approaching the } \\
\text { world. }\end{array}$ & $\begin{array}{l}\text { Deku } \\
\text { Tree, Navi,Link }\end{array}$ & $\begin{array}{l}\text { Deku } \\
\text { Tree }\end{array}$ & $\beta$ & & $\begin{array}{l}\text { Meeting } \\
\text { with the } \\
\text { Mentor }\end{array}$ \\
\hline $\begin{array}{l}\text { Character- } \\
\text { Driven }\end{array}$ & Kernel & Event & $\begin{array}{l}\text { The deku tree tasks Link with } \\
\text { breaking the curse he is under. }\end{array}$ & $\begin{array}{l}\text { Deku } \\
\text { Tree,Link }\end{array}$ & $\begin{array}{l}\text { Deku } \\
\text { Tree }\end{array}$ & $\gamma$ & $\begin{array}{l}\text { The Call to } \\
\text { Adventure }\end{array}$ & $\begin{array}{l}\text { Call to } \\
\text { Adventure }\end{array}$ \\
\hline $\begin{array}{l}\text { Character- } \\
\text { Driven }\end{array}$ & Kernel & Main & $\begin{array}{l}\text { After Link defeats the curse, the } \\
\text { deku tree implores Link to stop } \\
\text { the evil man who set the curse } \\
\text { from gaining the Triforce. }\end{array}$ & $\begin{array}{l}\text { Deku } \\
\text { Tree, Link }\end{array}$ & $\begin{array}{l}\text { Deku } \\
\text { Tree }\end{array}$ & $\delta$ & $\begin{array}{l}\text { The Call to } \\
\text { Adventure }\end{array}$ & \\
\hline $\begin{array}{l}\text { Character- } \\
\text { Driven }\end{array}$ & Kernel & Chapter & $\begin{array}{l}\text { The Deku tree gives link the } \\
\text { Spiritual Stone of the Forest, and } \\
\text { tells Link to go to the castle and } \\
\text { meet the Princess. }\end{array}$ & $\begin{array}{l}\text { Deku } \\
\text { Tree,Link }\end{array}$ & $\begin{array}{l}\text { Deku } \\
\text { Tree }\end{array}$ & B & $\begin{array}{l}\text { Supernatural } \\
\text { Aid }\end{array}$ & \\
\hline Plot-Driven & Satellite & Event & $\begin{array}{l}\text { The deku tree dies despite Link's } \\
\text { assistance, too weak from the } \\
\text { curse. }\end{array}$ & Deku Tree & & & & \\
\hline $\begin{array}{l}\text { Character- } \\
\text { Driven }\end{array}$ & Satellite & Event & $\begin{array}{l}\text { On his way to the castle his friend } \\
\text { Saria says goodbye, and professes } \\
\text { their friendship. }\end{array}$ & Link,Saria & Saria & $\uparrow$ & $\begin{array}{l}\text { Crossing 1st } \\
\text { Threshold }\end{array}$ & $\begin{array}{l}\text { Crossing } \\
\text { 1st } \\
\text { Threshold }\end{array}$ \\
\hline
\end{tabular}




\section{Character-Driven or Plot-Driven}

The source of the event was determined and classified as either sourcing internally from a character or sourcing externally from the author. Character-sourced events were labelled character-driven, and author-sourced events were labelled plot-driven.

\section{Kernels and Satellites}

This categorization has to do with the importance of an event to the story. Satellites are story events which can be freely deleted from the discourse without affecting the overall structure and logic of the narrative. Kernels, on the other hand, are necessary to the narrative and cannot be omitted without changing it [19][3].

\section{Main, Chapter, Event}

"Main" events are derived from Glassner's definition of a story "arc" [1]. These events are central to the overarching plot or goal of the narrative. "Chapter" events are also turning points in the story, within the larger arc. Generally, in games, they correspond to a new level or the accomplishment of a minor goal and the switch to a new one. Remaining events are classified as "Events", and have no special properties in this categorization.

\section{Narrative Macrostructures}

Events were also categorized against three narrative macrostructures: Propp's Functions, The Hero's Journey, and the Writer's journey. If the event corresponded to a component or function of the macrostructure, it was labelled as such. 


\subsubsection{Visualizer Development for Preliminary Analysis}

Before we completed the entire study, we performed high-level pilot analysis of our early data points in order to clarify our research questions. To that end, we developed a visualizer that allowed us to more easily view patterns in the data.

The visualizer was developed as a simple web application using HTML and Javascript, altogether comprised of roughly 1000 lines of code. Web languages were chosen partly because of the researcher's familiarity and skillset with web tools and browser-based visualizations, but also because of data access tools offered by Google. Our data was stored in a Google Sheets spreadsheet, and Google offers a Visualization API which allows Javascript queries to Sheets data using a SQL-like syntax [51]. Every game in our data is stored as a different sheet within the master document. Google assigns every sheet a unique GID, which is used during the query to indicate which game data we wish to access. Visualizations are rendered using the browser canvas element.

The visualizer contains three visualization styles. These visualizations are the Arc, the Character Interactions, and the Character Co-Occurrence Matrix. Each visualization is described later in this section. While figures accompany each visualization description, Appendix 3 contains visualizations for each game in our dataset. 


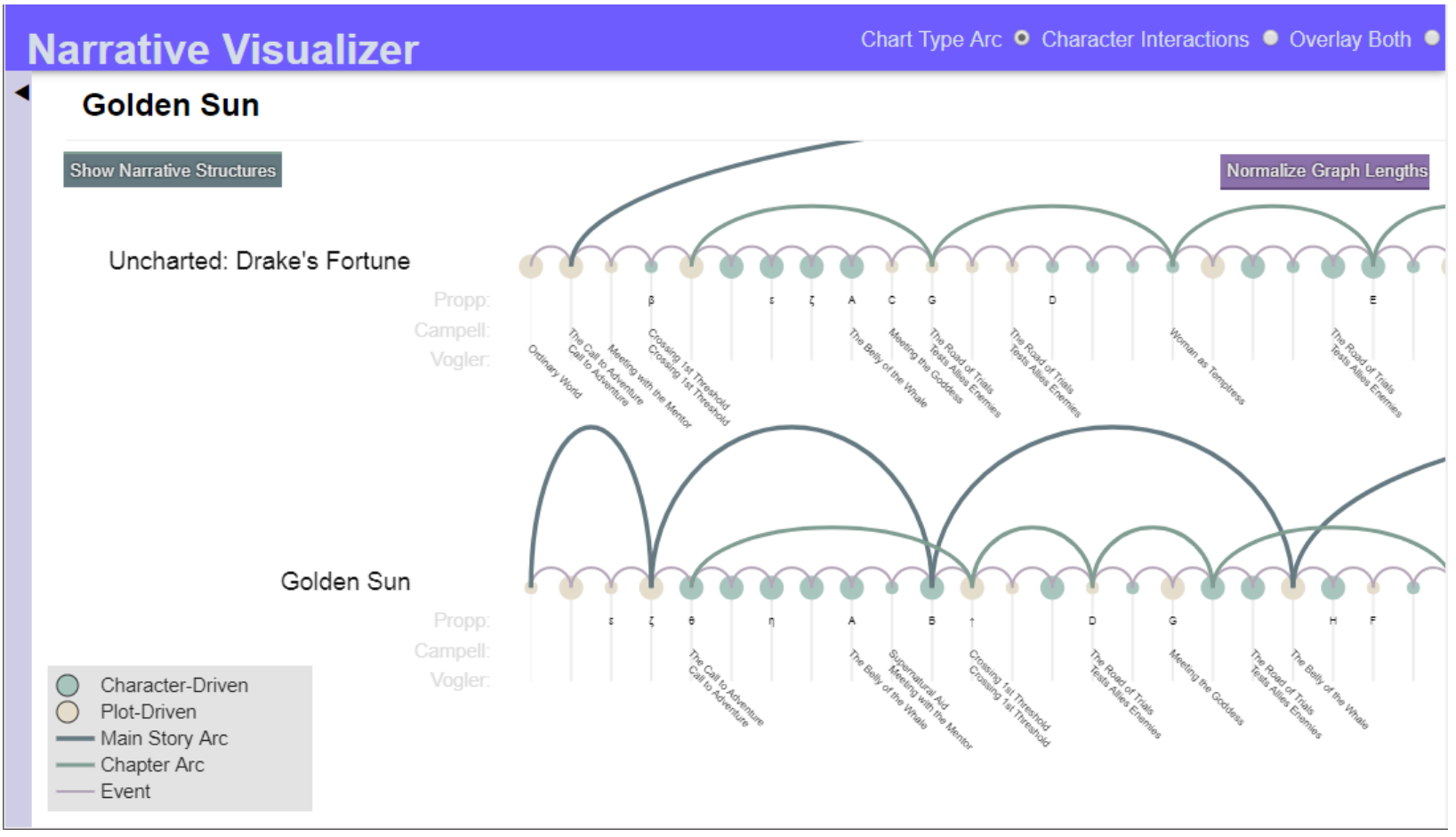

Figure 3: Narrative Visualizer interface for the Arc and Character Interactions visualizations. In this view the game selection list is collapsed. Two games have been selected to display.

The Arc and Character Interactions visualizations were developed concurrently. The overall interface for these two visualizers is shown in Figures 3 and 4 . These visualizations are event-based, meaning information is shown along a timeline for each event within the story. The Arc and Character Interactions visualizations are interactive. They can be zoomed in and out to see more detail, and more information about each story event can be accessed by hovering over the event node with the mouse. We additionally developed a feature to allow multiple game visualizations to be displayed at once, to better compare and view similarities and differences between games. Graph lengths can be visually normalized to compensate for different lengths in compared games. 


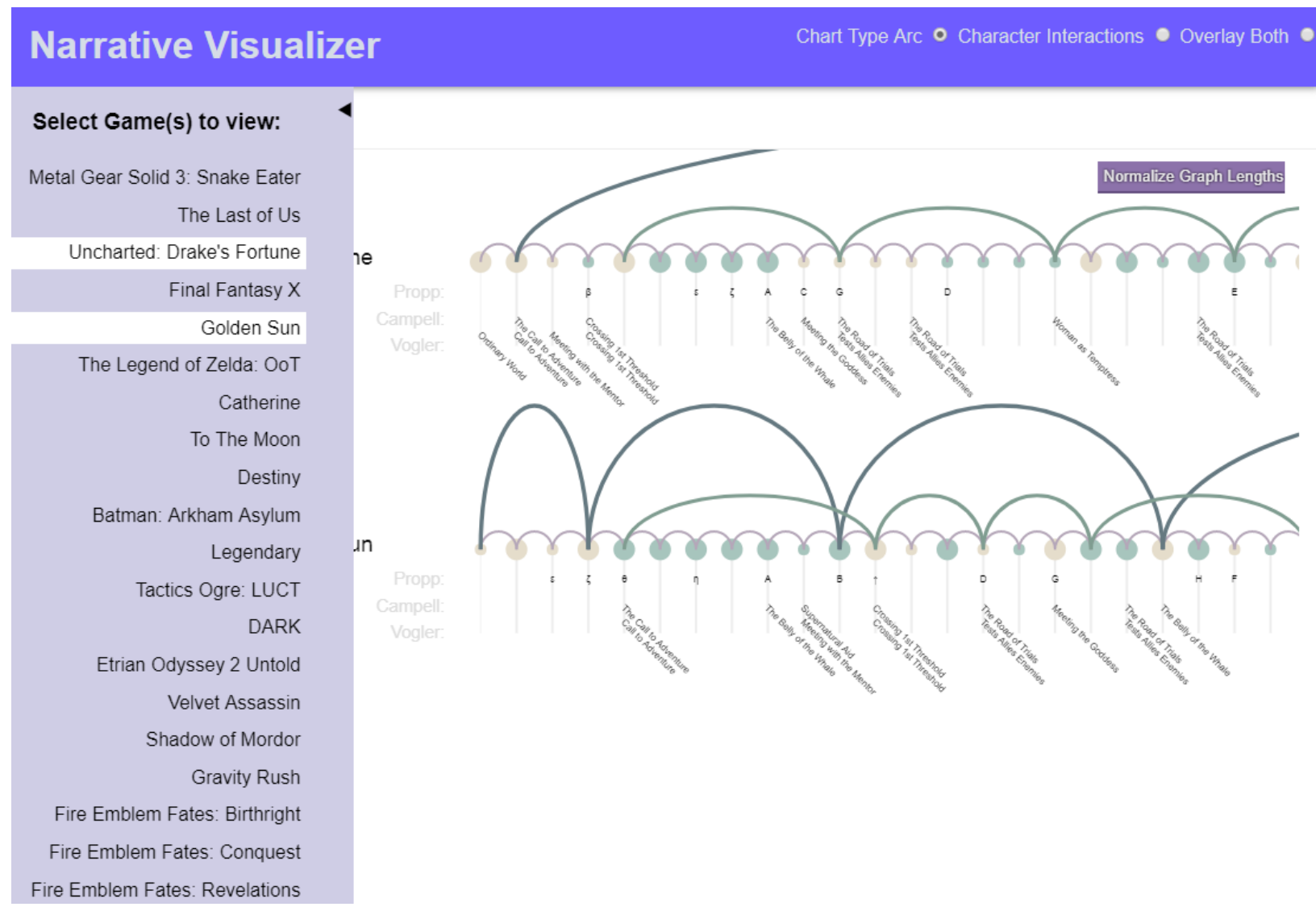

Figure 4: Narrative Visualizer interface, showing the game selection list. Multiple games can be selected, and selected games are highlighted as shown.

The Character Co-Occurrence Matrix was developed separately and does not feature the interactive or comparative features of the other visualizations. It is character-based, showing information about the story as it relates to the characters, and having no information corresponding to the individual events within the story.

The Arc Visualizer, shown in Figure 5, was meant to graphically show the main, chapter, and event levels as described in the previous section. The graph also has the option to display narrative macrostructure information, which would list macrostructure components which corresponded to that story event. Character-Driven and Plot-Driven events are distinguished by colour. The size of the node indicates whether it is a Kernel or a Satellite, with larger nodes 
being Kernels, and smaller nodes being Satellites. The hope with this visualization was to identify obvious patterns, such as a consistent amount of events per chapters. However, no useful conclusions or hypothesis were found from this visualization.

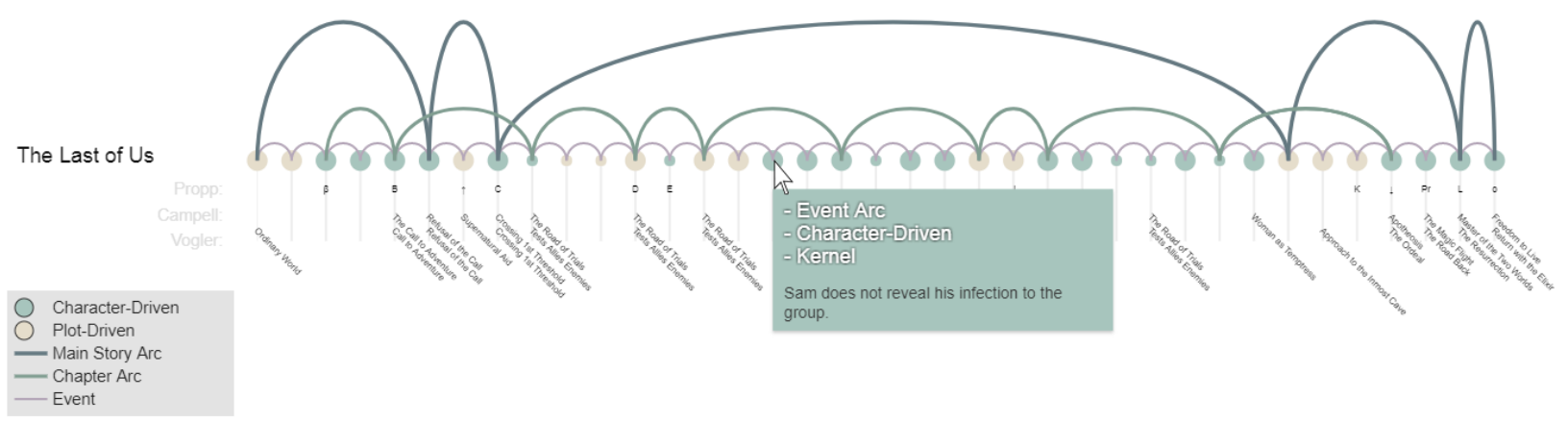

Figure 5. Arc Visualization of the story from "The Last of Us" video game.

The second visualization we developed is the Character Interactions visualization. Again, this is a timeline based visualization, with each event in the story appearing as a circular node along the horizontal access. Every character is delineated by a line on the graph, which is assigned a random colour to differentiate them. Whenever the character participated in a story event, their line would converge to the central axis at that event node. Character-driven and Plot-driven events were distinguished by the colour of the event node. Plot-driven events are drawn as black, while a character-driven event is coloured in the same colour as the character that "drove" the event.

We found that games with higher-rated stories tended to have more activity along the graph. These games would, on average, feature more characters, more character-driven events and characters participating in more events along the timeline. Lower scoring games featured fewer characters, more plot-driven events, and characters less frequently involved in story events. This contrast can be seen in Figure 6. 

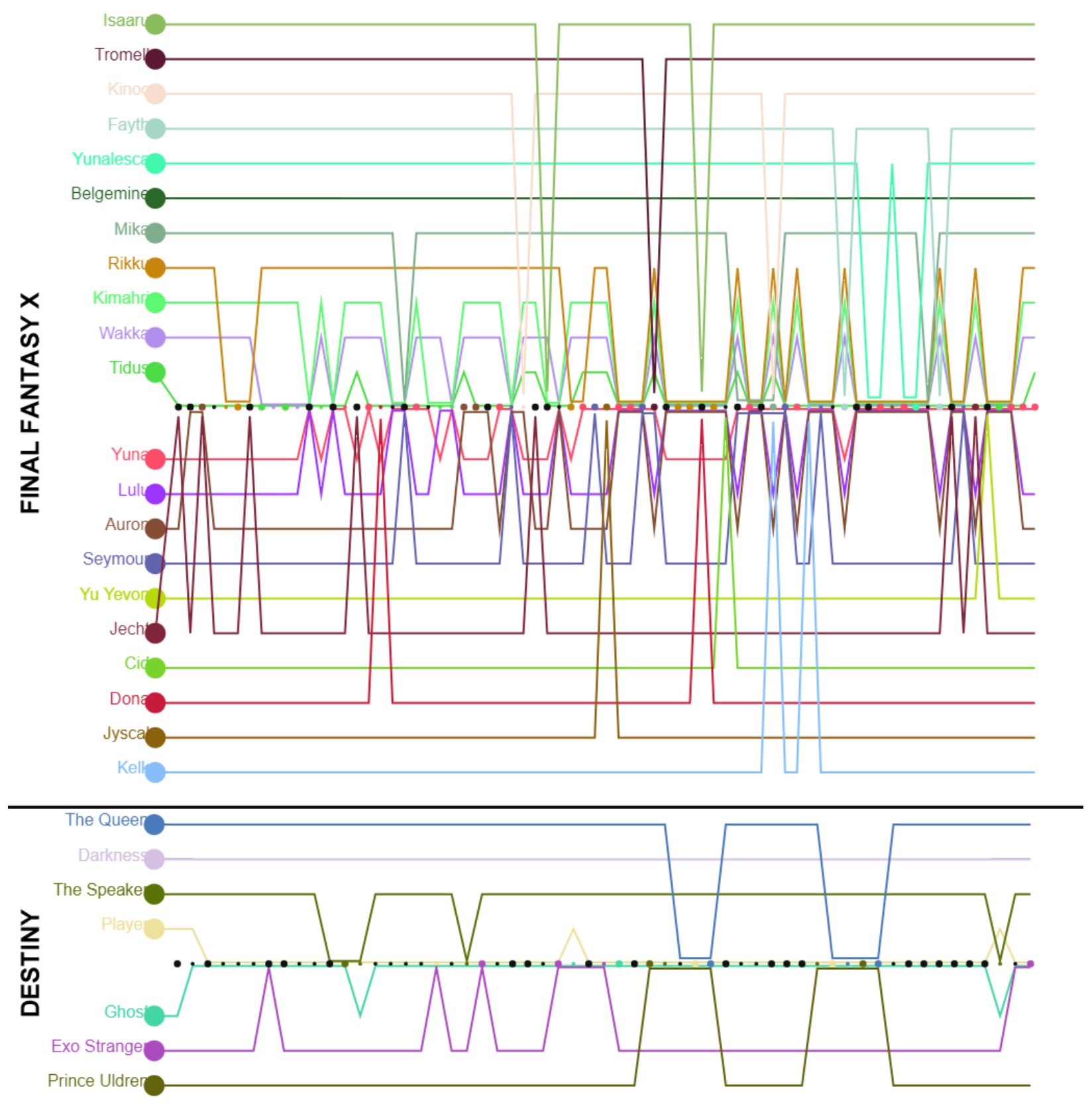

Figure 6. Character Interactions Visualization for Game Stories. The top visual shows a highly rated game story, while the bottom shows a low rated one.

We had initially hypothesized that our idea of distinguishing events as either characterdriven or plot-driven would provide useful information towards our model. This visualization showed us that our hypothesis had merit, and was worth further study and analysis. 
We developed a feature to overlay both of these timeline graphs overtop of one another (Figure 7), in order to see if there was any connection between the two variables. However, as mentioned, we found little usefulness in the main / chapter / event visualization. Therefore, no further insights were gained from this feature.

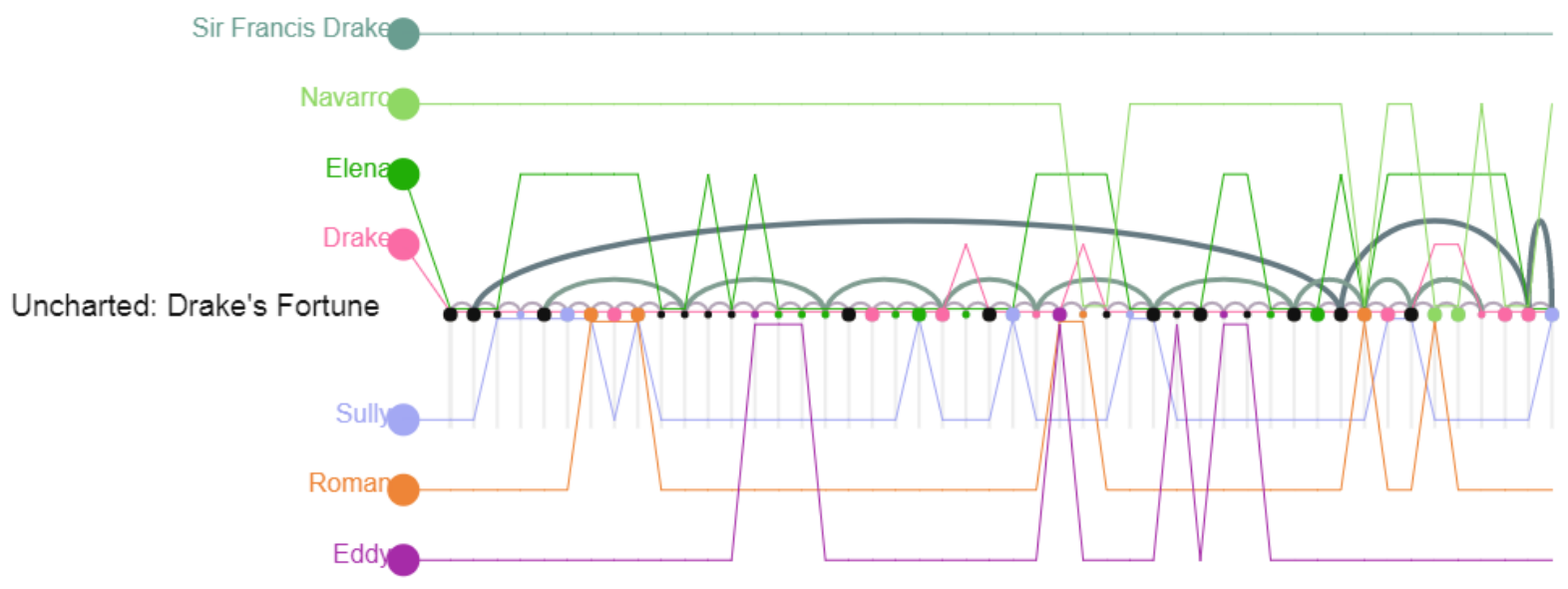

Figure 7: The Arc and Character Interactions visualizations overlaid for the game "Uncharted: Drake's Fortune".

The last visualization is the character co-occurrence matrix, shown in Figure 8. This diagram illustrates the importance of the various characters within the story based on how frequently they appear alongside other characters. The more frequently the characters cooccur, the darker the square is drawn. The exact count of events where the two characters interacted is also displayed. The total number of events in the game story is displayed at the right-hand side of the visualization. Again, as found with the Character Interactions Visualization, games with higher rated stories featured more characters, particularly with more characters interacting with the game's protagonist. 


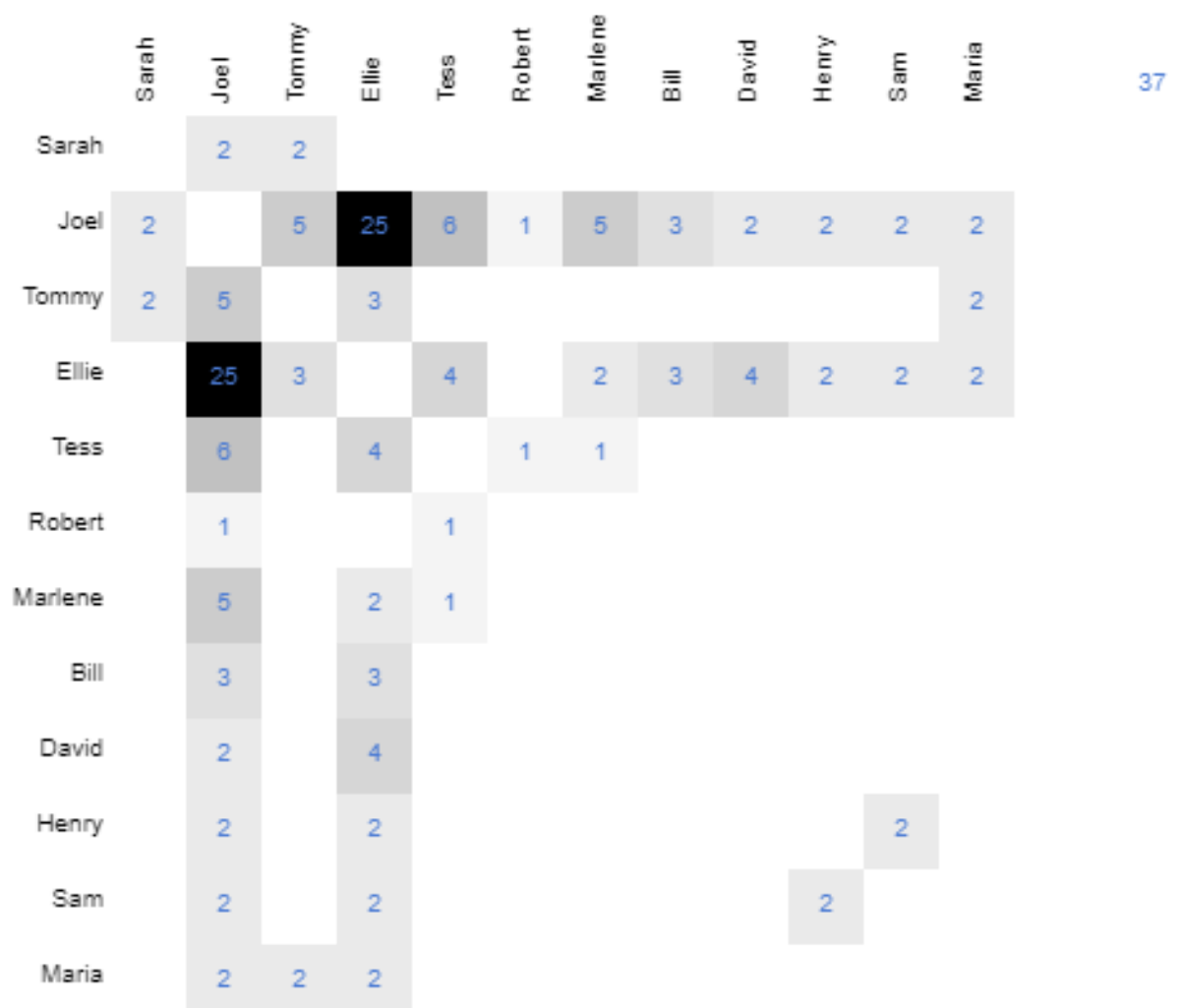

Figure 8. Character Co-occurrence Matrix for the story of "Metal Gear Solid 3".

To facilitate later analysis needs, a basic data-extractor tool was developed, also with HTML and Javascript. The purpose of this tool was to be a simplified single-query system, where the query and desired variables could be altered in the code, and then executed to quickly extract data in string form for use in various analyses. Again, the sheet GID is used to indicate which game we wish to query against. Figure 9 shows the extractor tool output. This tool was used to more quickly generate simplified data-sheets for use in our probability analyses as described in the following section. 


GiD: $1457986752 \quad$ Submit

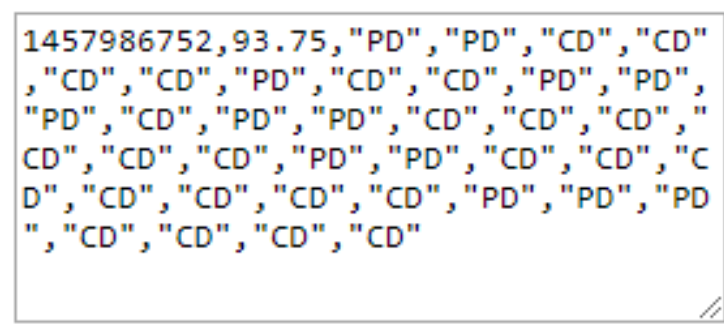

Figure 9: Data Extractor tool in use. In this image, the extractor is configured to generate a string listing the GID, the game score, and then an indication for each event in order as to whether it is character-driven, or plot-driven. 


\section{Results}

The full data-set consists of 20 analysed games. Results have been split into quantitative and qualitative categories. An overall summary of the games studied, and their aggregate critical score is listed in Table 2.

Table 2. Listing of analysed games and their aggregate critical scores.

\begin{tabular}{ll}
\hline Game Name & Score \\
\hline Dark & 41.67 \\
Legendary & 46.25 \\
Velvet Assassin & 59.38 \\
Destiny & 72.6 \\
Gravity Rush & 77.5 \\
EtrianOdyssey 2: Untold & 78.5 \\
To The Moon & 81.25 \\
Catherine & 82 \\
FE:Fates - Conquest & 83.5 \\
Uncharted: Drake's Fortune & 83.67 \\
Shadow of Mordor & 85.17 \\
FE:Fates - Revelations & 86.25 \\
FE:Fates - Birthright & 87.9 \\
Final Fantasy X & 89.5 \\
Tactics Ogre: LUCT & 89.6 \\
Golden Sun & 91.67 \\
Batman: Arkham Asylum & 92 \\
The Last of Us & 93.75 \\
Metal Gear Solid 3 & 94.33 \\
Legend of Zelda: OoT & 96.25 \\
\hline
\end{tabular}




\subsection{Quantitative}

\subsubsection{Overall Results}

We calculated the percentage of events within each game that were character-driven and plotted this against the aggregate critical score (Figure 10). The graph suggests that there is a correlation between a higher percentage of character-driven events and a higher resultant score. Figure 11 shows a similar plot, performed on only Kernel events within the story. Again, we see that higher scoring games feature more character-driven Kernel events. For this initial analysis, percentages were used to try and reduce bias between longer and shorter stories. Story lengths vary widely, with some stories containing fewer than 50 story events, while others feature over 100 .

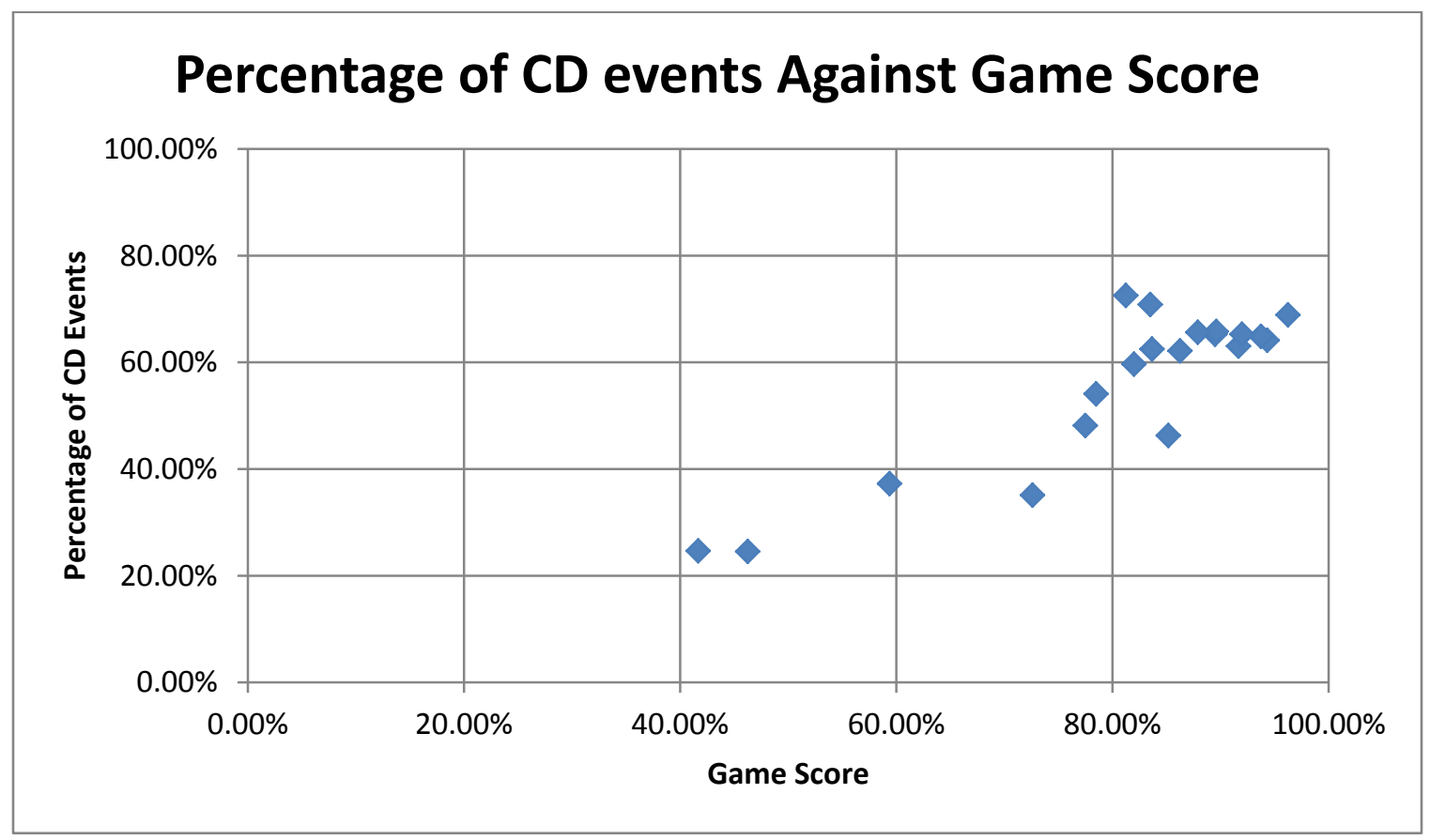

Figure 10. Percentage of Character-Driven events against Game Score 


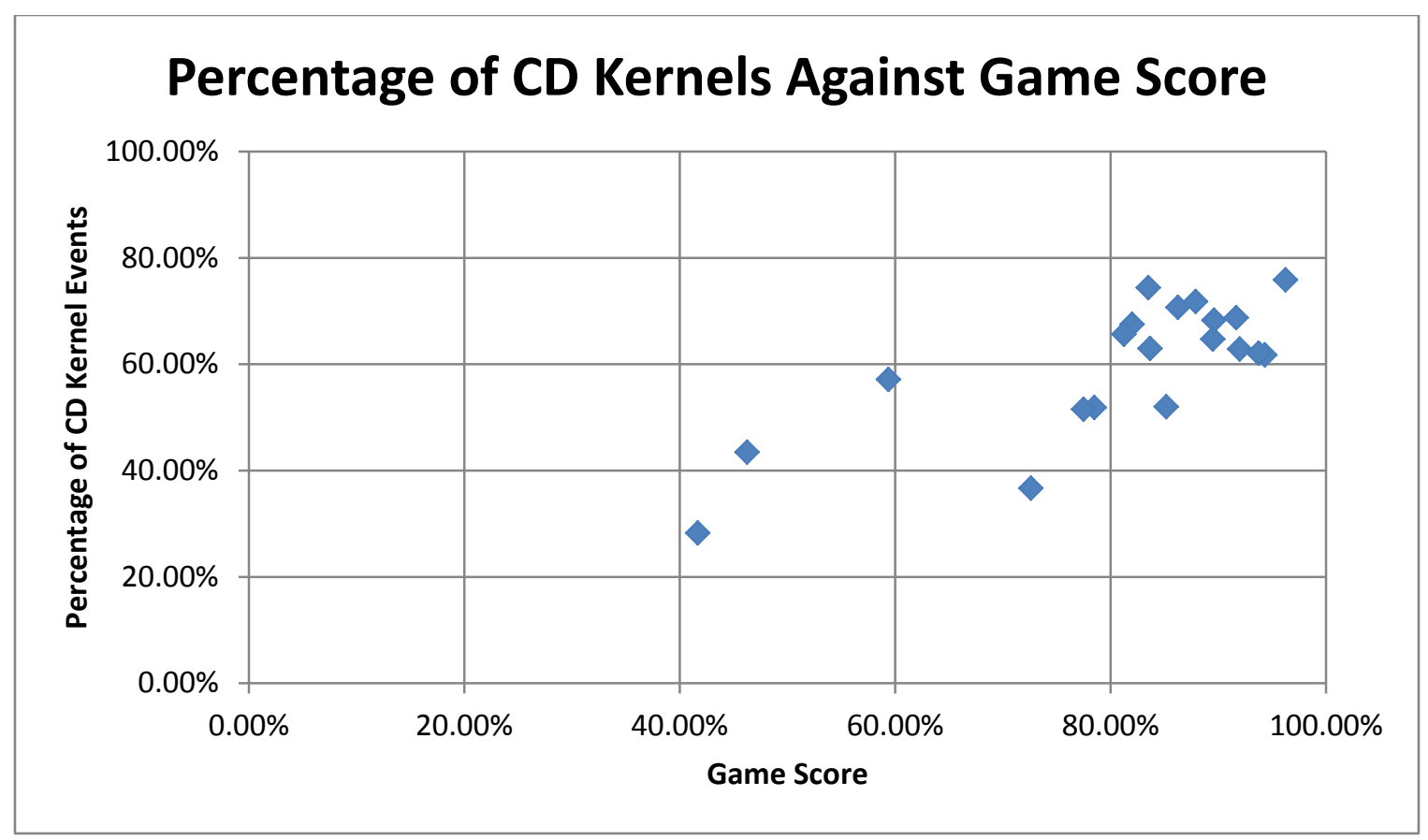

Figure 11. Percentage of kernel events which are Character-Driven plotted against game score

To confirm this correlation, we ran an ANOVA comparing the counts of CD and PD

events for each game to its score (Table 3). Our dataset, though it appears not fully normal, is close to normal because it was selected from random reviewers, and therefore suitable for an ANOVA. The results indicate that the number of PD and CD events and the interaction between them does affect the resultant game score.

Table 3. Analysis of Variance of the count of CD and PD events, related to the game Score

\begin{tabular}{llllll}
\hline & Df & Sum Sq & Mean Sq & F Value & $\operatorname{Pr}(>\mathrm{F})$ \\
\hline Count PD & 1 & 1908.7 & 1908.7 & 41.133 & $8.58 \mathrm{e}-06^{* * *}$ \\
Count CD & 1 & 1460.6 & 1460.6 & 31.475 & $3.91 \mathrm{e}-05^{* * *}$ \\
Count PD:CountCD & 1 & 257.4 & 257.4 & 5.547 & $0.0316^{*}$ \\
\hline
\end{tabular}

Residuals $\quad 16 \quad 742.5 \quad 46.4$ 


\subsubsection{Character-Driven and Plot-Driven Probability Analysis}

One of our goals is to be able to distinguish between better and worse stories within the model. To support this, a median split was done on the data, splitting the data into lower scoring and higher scoring cohorts. However, our data set is skewed towards higher scoring games, with a fairly high overall median of 84.42 . The high median meant that much of the lower half of the data would still score high enough to be considered "good" as opposed to "bad", making it difficult to compare the two halves and make conclusions. Therefore, in addition to the median split, the data was also split into lower and upper thirds, with the middle third being ignored. The lower third contained games scoring from 41.67 - 81.25, while the upper third ranged from 89.5-96.25. A summary of the Median and Mean scores from these dataset splits can be found in Table 4. These values confirm that the overall dataset is "topheavy", given that the medians and means in the upper half and upper third are much closer together than what is seen in the lower half and lower third.

Table 4. Summary of Median and Mean scores when the data set is split

\begin{tabular}{lll}
\hline & Median Score & Mean Score \\
\hline Overall & 84.42 & 80.64 \\
Lower Half & 78 & 70.632 \\
Upper Half & 90.64 & 90.64 \\
Lower Third & 72.6 & 65.31 \\
Upper Third & 92 & 92.44 \\
\hline
\end{tabular}

We analyzed the probabilities of moving between Character-driven and Plot-driven events from these datasets. A graphical representation of this system is shown in Figure 12. 
Probabilities were also measured for strings of events, up to a string of 5. (Example, C-C, CC-P, CPC-C, and so on). We found that this information becomes less useful beyond a string of 3 events, as data numbers begin to thin out and probabilities become unreliable.

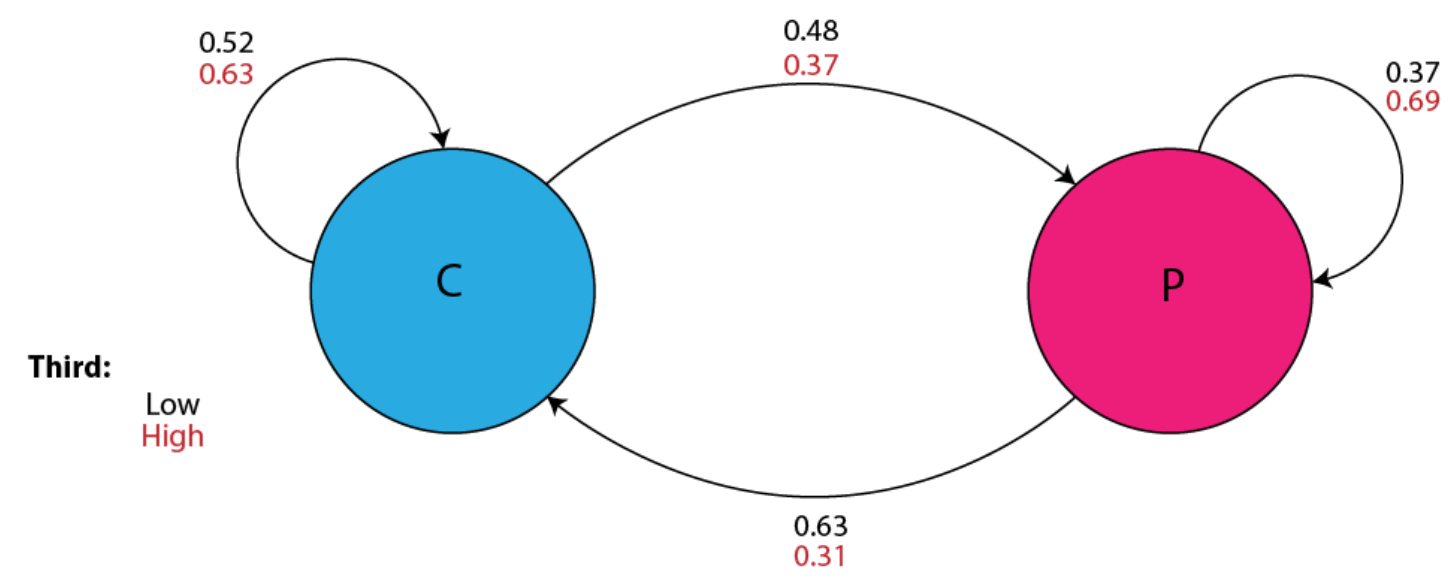

Figure 12: Markhov State Machine modelling the movement between character-driven and plot-driven events, contrasted between the lower and upper thirds of the data set

Table 5 shows the probability data for 2-length strings of events. Binomial tests were run to determine if the observed probability values were significantly different from a neutral $50 \%$ value. In the unsplit dataset, character-driven events were found to occur significantly higher than $50 \%$ of the time when following a character-driven event, with a p-value of $<0.0001$. That is, C-C strings were more probable than C-P strings. Plot-driven events were not significantly more likely to follow plot-driven events (P-P string), with a p-value of 0.103. In the lower half of the data set, character-driven events were more likely to follow both character and plot-driven events, with $p$ values of $<0.0001$ (C-C string) and 0.00259 ( $\mathrm{P}-\mathrm{C}$ string) respectively. 
In order to determine if differences could be identified between the split data-sets, a Chi-Squared comparison was run on the character and plot-driven event counts (Table 6). Median High was compared with Median Low, and Upper Third was compared with Low Third.

Table 5. Observed Probability values for 2-tuple event strings

\begin{tabular}{llllll}
\hline & \multicolumn{5}{c}{ Probability } \\
\cline { 2 - 6 } & Overall & Lower Half & Upper Half & Lower Third & Upper Third \\
C-C & 0.60 & 0.59 & 0.61 & 0.52 & 0.63 \\
C-P & 0.40 & 0.41 & 0.39 & 0.48 & 0.37 \\
P-P & 0.53 & 0.42 & 0.67 & 0.37 & 0.69 \\
P-C & 0.47 & 0.58 & 0.33 & 0.63 & 0.31 \\
\hline
\end{tabular}

Table 6. Chi-Squared Comparison of the Median and Third split datasets for 2 tuple event strings

\begin{tabular}{llll}
\hline Testing Proportion: & Chi-Squared & DF & P \\
\hline C-C Median High vs Median Low & 0.315 & 1 & 0.5745 \\
P-P Median High vs Median Low & 34.538 & 1 & $<0.0001$ \\
C-C High Third vs Low Third & 5.671 & 1 & 0.0172 \\
P-P High Third vs Low Third & 35.596 & 1 & $<0.0001$ \\
\hline
\end{tabular}

A significant difference was found between the proportion of events which follow a plot-driven event between the median high and median low datasets. There is also a significant difference between the proportions of events which follow both character and plot-driven events in the High Third and Low Third.

For 3-length event strings, the observed probability values are listed in Table 7, and a graphical representation is shown in Figure 13. 
Table 7. Observed Probability values for 3-tuple event strings

\begin{tabular}{|c|c|c|c|c|c|}
\hline & \multicolumn{5}{|c|}{ Probability } \\
\hline & Overall & Lower Half & Upper Half & Lower Third & Upper Third \\
\hline C-C & 0.63 & 0.62 & 0.65 & 0.53 & 0.66 \\
\hline C-P & 0.37 & 0.38 & 0.35 & 0.47 & 0.34 \\
\hline P-P & 0.63 & 0.55 & 0.69 & 0.51 & 0.71 \\
\hline P-C & 0.37 & 0.45 & 0.31 & 0.49 & 0.29 \\
\hline PC-C & 0.57 & 0.56 & 0.59 & 0.53 & 0.63 \\
\hline PC-P & 0.43 & 0.44 & 0.41 & 0.47 & 0.37 \\
\hline PP-C & 0.45 & 0.35 & 0.70 & 0.31 & 0.73 \\
\hline PP-P & 0.55 & 0.65 & 0.30 & 0.69 & 0.27 \\
\hline
\end{tabular}

Binomial tests were run to determine if the observed probability values from Table 7 were significantly different from a neutral $50 \%$ value (Table 8 ). Only events following a PP event chain did not differ significantly from $50 \%$.

Table 8. Binomial p-values for 3-tuple event strings. All Binomial tests were "Greater".

\begin{tabular}{ll}
\hline Event Chain & Binomial p-value \\
\hline CC-C & $<0.001$ \\
CP-C & $<0.001$ \\
PC-C & 0.005678 \\
PP-P & 0.07839 \\
\hline
\end{tabular}




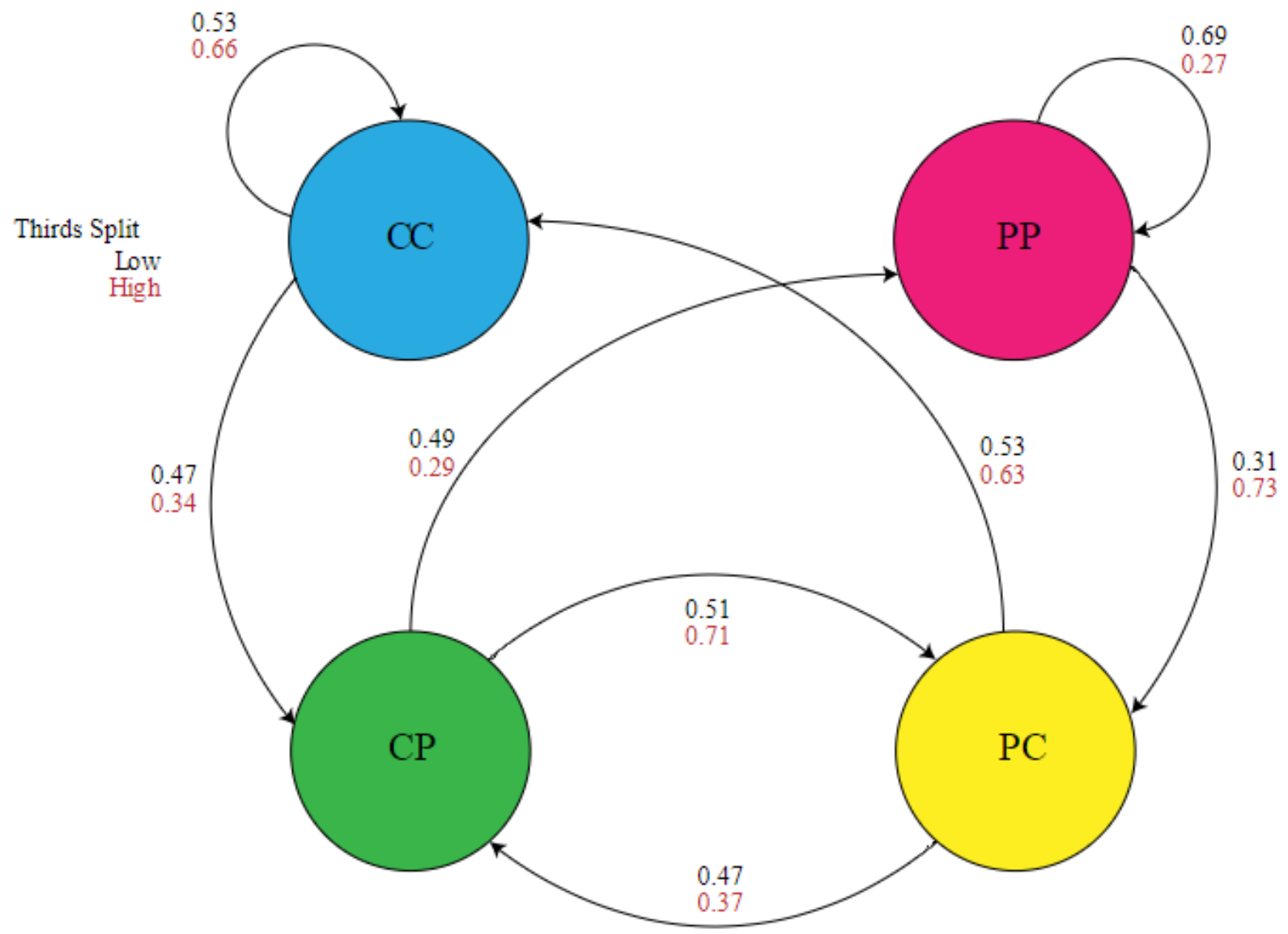

Figure 13: Markhov State Machine modelling the movement between character-driven and plot-driven events in 3tuple strings, contrasted between the lower and upper thirds of the data set.

Chi-Squared comparisons were run for the 3-tuple event strings, similarly to the 2-tuple comparisons (Table 9). When comparing the Median High and Median Low datasets, there is a significant difference between the proportion of character-driven events following CP and PP event chains. When the High Third and Low Third datasets are compared, there is a significant difference between the proportions of character-driven events which follow CC, CP, or PC event chains. 
Table 9. Chi-Squared Comparison of the Median and Third split datasets for 3 tuple event strings

\begin{tabular}{llll}
\hline Testing Proportion: & Chi-Squared & DF & P \\
\hline CC-C Median High vs Median Low & 0.434 & 1 & 0.5099 \\
CP-C Median High vs Median Low & 5.973 & 1 & 0.0145 \\
PC-C Median High vs Median Low & 0.275 & 1 & 0.5997 \\
PP-C Median High vs Median Low & 27.430 & 1 & $<0.0001$ \\
CC-C High Third vs Low Third & 4.527 & 1 & 0.0334 \\
CP-C High Third vs Low Third & 7.516 & 1 & 0.0061 \\
PC-C High Third vs Low Third & 1.929 & 1 & 0.1649 \\
PP-C High Third vs Low Third & 23.917 & 1 & $<0.0001$ \\
\hline
\end{tabular}

In 4-tuple and beyond event strings, as mentioned above, sample sizes deteriorate to the point where statistical analysis is not useful.

The overall probabilities of moving between character and plot events were run for every game individually. Some clear trends can be seen in all games. The number of PP-P event strings, for example, decreases rapidly as the game's score increases (Figure 14). Lower scoring games contain much more frequent and longer plot event strings when compared to higher scoring games. Interestingly, $95 \%$ of game stories within the dataset begin with a plot-driven event. 


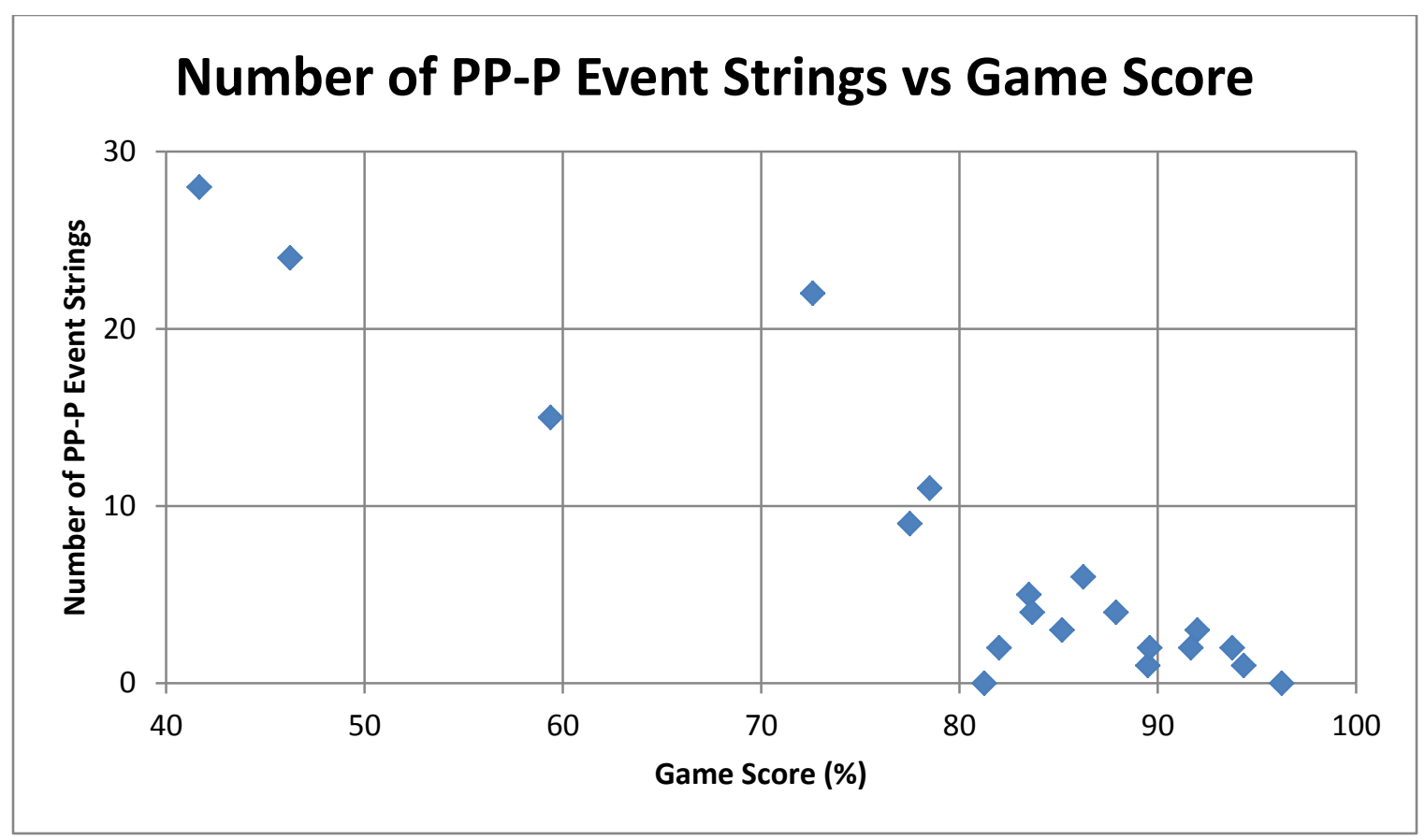

Figure 14: Number of PP-P Event Strings plotted against Game Score.

\subsubsection{Narrative Macrostructures}

We performed an of our data against the narrative macrostructures (The Hero's

Journey, The Writer's Journey, and Propp's Functions). For the following analysis, the frequency of each function within the data was found, alongside the frequency with which each function was a character-driven or a kernel event. A binomial test was run to see if the frequency of the character-driven or kernel events differed significantly from $50 \%$. The following tables show the results for each macrostructure. Results that are significant are highlighted. A "greater" binomial test was used, indicating that for significant events, the probability of being the given event-type (either CD or Kernel), was significantly greater than $50 \% . A *$ beside the value indicates that a "lesser" binomial test was used instead. A dash indicates the test was not run due to inadequate sample size. 
Table 10. The Hero's Journey Structural Data within the Dataset

\begin{tabular}{lllllll}
\hline Function & Count & CD & Kernel & $\begin{array}{l}\text { Occurrence } \\
\text { Per Game }\end{array}$ & $\begin{array}{l}\text { Binom } p \\
\text { value (CD) }\end{array}$ & $\begin{array}{l}\text { Binom } p \\
\text { value } \\
\text { (Kernel) }\end{array}$ \\
\hline The Call to Adventure & 25 & 12 & 23 & 1.25 & 0.5 & 0.04477 \\
Refusal of the Call & 8 & 6 & 3 & 0.40 & 0.1445 & 0.3633 \\
Supernatural Aid & 14 & 5 & 9 & 0.70 & $0.212^{*}$ & 0.212 \\
Crossing 1st Threshold & 19 & 15 & 12 & 0.95 & 0.009605 & 0.1796 \\
The Belly of the Whale & 21 & 15 & 18 & 1.05 & 0.03918 & 0.0007448 \\
The Road of Trials & 162 & 95 & 70 & 8.10 & 0.01679 & $0.04933 *$ \\
Meeting the Goddess & 17 & 8 & 14 & 0.85 & $0.5 *$ & 0.006363 \\
Woman as Temptress & 26 & 23 & 19 & 1.30 & $<0.0001$ & 0.01448 \\
Atonement with the Father & 18 & 16 & 14 & 0.90 & 0.0006561 & 0.01544 \\
Apotheosis & 19 & 15 & 14 & 0.95 & 0.009605 & 0.03178 \\
Ultimate Boon & 17 & 9 & 14 & 0.85 & 0.5 & 0.006363 \\
Refusal of the Return & 7 & 6 & 5 & 0.35 & 0.0625 & 0.2266 \\
The Magic Flight & 2 & 1 & 1 & 0.10 & - & - \\
Rescue from Without & 2 & 1 & 2 & 0.10 & - & - \\
Crossing the Return Threshold & 6 & 6 & 5 & 0.30 & 0.01563 & 0.1094 \\
Master of the Two Worlds & 13 & 7 & 10 & 0.65 & 0.5 & 0.04614 \\
Freedom to Live & 17 & 13 & 13 & 0.85 & 0.02452 & 0.02452 \\
\hline
\end{tabular}

Table 11. The Writer's Journey Structural Data within the Dataset

\begin{tabular}{|c|c|c|c|c|c|c|}
\hline Function & Count & $\mathrm{CD}$ & Kernel & $\begin{array}{l}\text { Occurrence } \\
\text { Per Game }\end{array}$ & $\begin{array}{l}\text { Binom } p \\
\text { value } \\
\text { (CD) }\end{array}$ & $\begin{array}{l}\text { Binom p } \\
\text { value } \\
\text { (Kernel) }\end{array}$ \\
\hline Ordinary World & 16 & 0 & 9 & 0.80 & $<0.0001$ & 0.4018 \\
\hline Call to Adventure & 22 & 11 & 20 & 1.10 & 0.5841 & $<0.0001$ \\
\hline Refusal of the Call & 7 & 6 & 2 & 0.35 & 0.0625 & 0.2266 \\
\hline Meeting with the Mentor & 15 & 8 & 10 & 0.75 & 0.5 & 0.1509 \\
\hline Crossing 1st Threshold & 20 & 16 & 12 & 1.00 & 0.005909 & 0.2517 \\
\hline Tests Allies Enemies & 168 & 99 & 74 & 8.40 & 0.01248 & $0.07123 *$ \\
\hline $\begin{array}{l}\text { Approach to the Inmost } \\
\text { Cave }\end{array}$ & 20 & 12 & 18 & 1.00 & 0.2517 & 0.0002012 \\
\hline The Ordeal & 19 & 16 & 17 & 0.95 & $2.21 \mathrm{E}-03$ & 0.0003643 \\
\hline Reward & 15 & 10 & 13 & 0.75 & 0.1509 & 0.003693 \\
\hline The Road Back & 10 & 7 & 6 & 0.50 & 0.1719 & 0.377 \\
\hline The Resurrection & 18 & 15 & 14 & 0.90 & 0.003769 & 0.01544 \\
\hline Return with the Elixir & 19 & 15 & 15 & 0.95 & 0.009605 & 0.009605 \\
\hline
\end{tabular}


Table 12. Structural Data for Propp's Functions within the Dataset

\begin{tabular}{|c|c|c|c|c|c|c|c|}
\hline Function & & Count & $C D$ & Kernel & $\begin{array}{l}\text { Occurrence } \\
\text { Per Game }\end{array}$ & $\begin{array}{l}\text { Binom p } \\
\text { value } \\
\text { (CD) }\end{array}$ & $\begin{array}{l}\text { Binom p } \\
\text { value } \\
\text { (Kernel) }\end{array}$ \\
\hline Absentation & $\beta$ & 11 & 7 & 9 & 0.55 & 0.2744 & 0.03271 \\
\hline Interdiction & $\gamma$ & 8 & 5 & 5 & 0.40 & 0.3633 & 0.3633 \\
\hline Violation & $\delta$ & 5 & 5 & 2 & 0.25 & 0.03125 & - \\
\hline Reconnaissance & $\varepsilon$ & 2 & 1 & 1 & 0.10 & - & - \\
\hline Delivery & $\zeta$ & 3 & 2 & 2 & 0.15 & - & - \\
\hline Trickery & $\eta$ & 2 & 2 & 1 & 0.10 & - & - \\
\hline Complicity & $\theta$ & 3 & 3 & 3 & 0.15 & - & - \\
\hline Villainy & A & 8 & 2 & 7 & 0.40 & 0.1445 & 0.03516 \\
\hline Lack & a & 7 & 5 & 5 & 0.35 & 0.2266 & 0.2266 \\
\hline $\begin{array}{l}\text { Mediation, The connective } \\
\text { incident }\end{array}$ & B & 17 & 9 & 16 & 0.85 & 0.5 & 0.0001373 \\
\hline Beginning Counteraction & C & 15 & 11 & 12 & 0.75 & 0.05923 & 0.01758 \\
\hline Departure & $\uparrow$ & 18 & 9 & 13 & 0.90 & 0.5927 & 0.04813 \\
\hline The First Function of the Donor & $\mathrm{D}$ & 23 & 15 & 17 & 1.15 & 0.105 & 0.01734 \\
\hline The Hero's Reaction & $\mathrm{E}$ & 13 & 11 & 9 & 0.65 & 0.01123 & 0.1334 \\
\hline $\begin{array}{l}\text { Provision / Receipt of a } \\
\text { Magical Agent }\end{array}$ & $\mathrm{F}$ & 20 & 11 & 15 & 1.00 & 0.4119 & 0.02069 \\
\hline Spatial Transference, Guidance & G & 17 & 6 & 13 & 0.85 & 0.1662 & 0.02452 \\
\hline Struggle & $\mathrm{H}$ & 15 & 7 & 8 & 0.75 & 0.5 & 0.5 \\
\hline Branded & J & 10 & 6 & 8 & 0.50 & 0.377 & 0.05469 \\
\hline Victory & I & 17 & 8 & 15 & 0.85 & 0.5 & 0.001175 \\
\hline Initial Lack is Liquidated & $\mathrm{K}$ & 15 & 10 & 14 & 0.75 & 0.1509 & 0.0004883 \\
\hline Return & $\downarrow$ & 4 & 3 & 2 & 0.20 & - & - \\
\hline Pursuit, Chase & $\operatorname{Pr}$ & 5 & 2 & 3 & 0.25 & - & - \\
\hline Rescue & Rs & 2 & 1 & 2 & 0.10 & - & - \\
\hline Unrecognized Arrival & o & 3 & 1 & 2 & 0.15 & - & - \\
\hline Unfounded Claims & L & 3 & 3 & 3 & 0.15 & - & - \\
\hline Difficult Task & $M$ & 9 & 7 & 8 & 0.45 & 0.08984 & 0.01953 \\
\hline Solution & $\mathrm{N}$ & 4 & 4 & 4 & 0.20 & 0.0625 & 0.0625 \\
\hline Recognition & Q & 5 & 3 & 3 & 0.25 & - & - \\
\hline Exposure & Ex & 8 & 7 & 7 & 0.40 & 0.03516 & 0.03516 \\
\hline Transfiguration & $\mathrm{T}$ & 13 & 9 & 10 & 0.65 & 0.1334 & 0.04614 \\
\hline Punishment & $U$ & 5 & 2 & 4 & 0.25 & - & - \\
\hline Wedding & W & 12 & 6 & 8 & 0.60 & 0.6128 & 0.1938 \\
\hline
\end{tabular}


When the data was split into thirds, sample sizes were too small to gain meaningful test data from most macrostructure elements. We were, therefore, unable to perform comparisons between high and low scoring games and how the narrative macrostructures apply differently.

With Propp's Functions, many functions were present so rarely that statistical analysis couldn't be done on the overall dataset. Very few functions significantly differed from $50 \%$ for $C D / P D$, however, many functions were significantly higher than $50 \%$ chance to be a kernel.

\subsubsection{Conformance to Macrostructures}

As part of our analysis, we looked at the occurrence of each function from our chosen macrostructures in order to verify whether they are a valid macrostructure for a video game narrative. The number of times the function appeared in the data set was counted and divided among the total number of games to create an "occurrence per game" metric. This data is listed in Tables 10, 11, and 12 previously.

The best fitting narrative macrostructure is the Writer's Journey. Two of the 12 functions, the "Refusal of the Call" and "The Road Back", have an occurrence per game of 0.5 or less. Otherwise, all functions within The Writer's Journey are well represented. The Call to Adventure, Crossing the $1^{\text {st }}$ Threshold, Tests Allies Enemies, and Approach to the Inmost Cave all have occurrences per game of 1.0 or higher.

For The Hero's Journey, five of the 17 functions had an occurrence per game of 0.5 or less (Refusal of the Call, Refusal of the Return, The Magic Flight, Rescue from Without, Crossing the Return Threshold). Therefore, while the Hero's Journey is not as good of a fit as the Writer's Journey, the bulk of the structure is still present in most game stories that were analysed. 
Propp's functions did not appear as consistently within the dataset. However, Propp himself wrote that all 31 functions were not required to be present in order to create a story, just that events within stories could all be classified to these 31 functions [5]. However, the large variance in occurrence per game between the functions, as well as the low number of functions appearing in most stories, leads us to conclude that this macrostructure is not as useful for generation as the Hero's and Writer's Journeys.

\subsubsection{Critical Reviews}

As part of the data collection, for every critical review, if there was a particularly positive or negative opinion about the game's story this was recorded. In general, we found that games with lower scores had more negative story mentions in their reviews, while games with higher scores had more positive story mentions. A summary of our findings is listed in Table 14. 
Table 13. Number of positive and negative opinions about game stories within their critical review aggregate. Note that some reviews had both positive and negative story mentions within the same review.

\begin{tabular}{|c|c|c|c|c|}
\hline Game Name & Score & $\begin{array}{l}\text { Number of } \\
\text { Reviews }\end{array}$ & $\begin{array}{l}\text { Positive Story } \\
\text { Mentions }\end{array}$ & $\begin{array}{l}\text { Negative Story } \\
\text { Mentions }\end{array}$ \\
\hline Dark & 41.67 & 3 & & 3 \\
\hline Legendary & 46.25 & 4 & & 2 \\
\hline Velvet Assassin & 59.38 & 4 & & 3 \\
\hline Destiny & 72.6 & 5 & & 5 \\
\hline Gravity Rush & 77.5 & 4 & 1 & 1 \\
\hline EtrianOdyssey 2: Untold & 78.5 & 4 & & 2 \\
\hline To The Moon & 81.25 & 4 & 4 & \\
\hline Catherine & 82 & 5 & 5 & \\
\hline FE:Fates - Conquest & 83.5 & 5 & 2 & 2 \\
\hline Uncharted: Drake's Fortune & 83.67 & 3 & 2 & \\
\hline Shadow of Mordor & 85.17 & 3 & 1 & 2 \\
\hline FE:Fates - Revelations & 86.25 & 4 & 3 & 3 \\
\hline FE:Fates - Birthright & 87.9 & 5 & 5 & \\
\hline Final Fantasy $\mathrm{X}$ & 89.5 & 4 & 3 & \\
\hline Tactice Ogre: LUCT & 89.6 & 5 & 5 & 1 \\
\hline Golden Sun & 91.67 & 3 & 3 & \\
\hline Batman: Arkham Asylum & 92 & 4 & 2 & \\
\hline The Last of Us & 93.75 & 4 & 3 & \\
\hline Metal Gear Solid 3 & 94.33 & 3 & 2 & 1 \\
\hline Legend of Zelda: OoT & 96.25 & 4 & 3 & \\
\hline
\end{tabular}

\subsubsection{Distance Analysis between Games}

We performed a Hamming Distance analysis comparing each game to every other game to see if we could gain any insights about the ordering of events. We parsed the data for each game into a long string made up of " $C D$ " and "PD" characters, and compared these strings to 
each other. Since the story strings are all different lengths, we employed several different truncation methods to see what results could be achieved:

1. No Truncation: Neither string was truncated, and every character position beyond the length of the shorter story was added to the Hamming Distance

2. End Truncation: We truncate at the end of the first input string, whether it be longer or shorter than the comparison string.

3. Middle Truncation: We compare characters alternating between the beginning position and the end position. In other words, we would first compare the first event from story 1 to the first event from story 2. Next, we compare the last event from story 1 to the last event from story 2, disregarding the length differences. Next, the 2 nd event from both stories would be compared. Eventually, all the events from the shorter string would have been compared. Remaining middle story events in the longer story were truncated.

$\mathrm{N}$-Squared matrices were created for all of these truncations. Table 13 shows the N-Squared matrix for the middle truncation to illustrate the data collected by this analysis.

We ultimately determined that no truncation and end truncation are not fair comparisons because critical story events tend to happen at these times. Mid truncation is perhaps the fairest comparison of the three, due to a lesser likelihood of key events being truncated. However, given the differing lengths of stories, and given that we could not guarantee similar events were being truncated, we ultimately decided that the Hamming Distance is not an effective tool for viewing the differences between two story strings. 
Table 14. Hamming Distance Matrix for Mid-Truncation

\begin{tabular}{|c|c|c|c|c|c|c|c|c|c|c|c|c|c|c|c|c|c|c|}
\hline & 1 & 23 & 22 & 34 & 36 & 27 & & & & & & 35 & 39 & 47 & 27 & 39 & 143 & \\
\hline & 0 & 23 & 19 & 24 & 263 & 31 & 2 & 30 & & & 20 & 23 & 23 & 28 & 23 & 30 & 163 & \\
\hline & 23 & 0 & 21 & 20 & 25 & 28 & 29 & & 25 & 16 & 25 & 26 & 27 & 32 & 23 & 302 & 262 & \\
\hline & 19 & 21 & 0 & 25 & & & & & & & 29 & 30 & 27 & 31 & & 33 & 183 & \\
\hline & 24 & 20 & 25 & 0 & 422 & & 25 & & 20 & 39 & 38 & 35 & 35 & 32 & 25 & 372 & 242 & \\
\hline & 26 & 25 & 28 & 42 & 02 & & & & 14 & & 53 & 49 & 30 & 40 & 20 & 31 & 152 & \\
\hline & 31 & 28 & 29 & 26 & 23 & 0 & 27 & 24 & 19 & 30 & 23 & 20 & 21 & 24 & 212 & 26 & 152 & 25 \\
\hline & 27 & 29 & 30 & 25 & 22 & 27 & 0 & 24 & 18 & 27 & 25 & 24 & 21 & 27 & 17 & 25 & 132 & \\
\hline & 30 & 24 & 28 & 35 & 42 & 24 & 24 & 0 & 26 & 44 & 34 & 28 & 29 & 33 & 172 & 29 & 162 & 21 \\
\hline & 26 & 25 & 26 & 20 & 14 & 19 & 18 & 26 & 0 & 26 & 20 & 24 & 23 & 20 & 22 & 21 & 152 & \\
\hline & 26 & 16 & 31 & 39 & 37 & 30 & 27 & 44 & 26 & 0 & 39 & 43 & 41 & (5) & 24 & 40 & 192 & \\
\hline & 20 & 25 & 29 & & & & 25 & 34 & 20 & 39 & 0 & 31 & 30 & 32 & 17 & 33 & 102 & \\
\hline & 23 & 26 & 30 & & & & 24 & 28 & 24 & 43 & 31 & 0 & 20 & 30 & 19 & 36 & 152 & \\
\hline & 23 & 27 & 27 & 35 & 302 & 21 & 21 & 29 & 23 & 41 & 30 & 26 & 0 & 31 & 15 & 31 & 112 & 26 \\
\hline & 28 & 32 & 31 & 32 & 402 & 24 & 27 & 33 & 20 & 39 & 32 & 30 & 31 & 0 & 213 & 34 & 162 & 25 \\
\hline & 23 & 23 & 31 & 25 & 202 & 21 & 17 & 17 & 22 & 24 & 17 & 19 & 15 & 21 & 0 & 20 & 15 & 16 \\
\hline & 30 & 30 & 33 & & 312 & 26 & 25 & 29 & 21 & 10 & 33 & 36 & 31 & 34 & 20 & 0 & 172 & 25 \\
\hline & 16 & 26 & 18 & & 15 & 15 & 13 & 16 & 15 & 19 & 15 & 15 & 11 & 16 & 15 & 17 & 01 & 17 \\
\hline & 33 & 25 & & & & & & & 26 & 27 & 27 & 24 & 26 & 25 & 162 & 25 & 17 & 0 \\
\hline & & & & & & & & & & & & 1 & & & 20 & & & 2 \\
\hline
\end{tabular}

\subsection{Qualitative Results}

\subsubsection{Internal and External Goals}

Appendix 4 contains an analysis of the internal and external goals as defined by Cron [2] of the protagonist(s) of each story. To review, internal goals are the true emotions or desires of the character, which are abstract and not associated with any particular action. External goals are the actions or goals they strive to complete in their quest to fulfil their internal goals. 
The 5 lowest scoring games in the data consistently have unclear internal character goals, as well as less relevant plot-driven events. However, this is not the case in the opposite direction. In The Legend of Zelda: Ocarina of Time, the highest scoring game in the data, while Link does have internal motivations, the plot-driven events are far more related to external goals than his internal ones.

\subsubsection{Discourse Rearrangement}

In our data, we noted that many games utilized discourse rearrangement to their benefit. That is, many games told their stories out of linear order, in order to strengthen the story or the emotional impact. An overview of these rearrangements and their effect on the narrative is given in Table 15. 
Table 15. Summary of Discourse Rearrangement styles found in our data, with discussion as to how it affected the overall outcomes of the narrative.

\begin{tabular}{ll}
\hline Game Title & Description of Discourse Arrangement \\
\hline The Legend of Zelda: Ocarina of & $\begin{array}{l}\text { Link is able to travel back and forth in-time to two periods 7 years } \\
\text { apart. Some child-timeline events take place later in the story than } \\
\text { adult-timeline events, even though the adult-timeline is } 7 \text { years later } \\
\text { chronologically. } \\
\text { Uses a dual-discourse structure. The overall narrative runs in a } \\
\text { forward temporal direction, but Johnny's memories are traversed in } \\
\text { reverse, with his most recent memories being explored at the start of } \\
\text { the game, and his older and eventually childhood memories towards } \\
\text { the end. } \\
\text { The major gameplay missions are actually Violet's memories from the } \\
\text { past. The current game narrative involves Violet much later than all } \\
\text { these memories, revisiting them in her mind. The game culminates in } \\
\text { a mission which takes place at "the present". } \\
\text { The game generally executes in chronological order, but there is also } \\
\text { a separate mini-discourse from the past which is revealed piece by } \\
\text { piece as Wraith's memories are discovered through progressing the } \\
\text { game. Wraith's past story influences the current story. } \\
\text { Bertrand experienced events } 100 \text { years prior to the story of the game. } \\
\text { Initially, this is not revealed to the player, but over time these events } \\
\text { are revealed and influence what he and other characters do in the } \\
\text { present. } \\
\text { The game generally operates chronologically but begins with a } \\
\text { powerful scene which actually occurs towards the end of the game. } \\
\text { Tidus' narration comes from the perspective of himself at the end of } \\
\text { the game, vs where he actually is at the current point of the game. } \\
\text { Additionally, memories from Tidus' past are periodically interspersed } \\
\text { to give context to his relationship with his father and affect how the } \\
\text { audience interprets his actions and emotions. }\end{array}$ \\
\end{tabular}




\section{Discussion}

This chapter presents our findings based on our study results. We respond to our research questions and discuss potential sources of error within our methodology. We then discuss potential applications for our research, both inside and out of academia.

\subsection{Research Question Response}

With this research, we hoped to determine whether a successful story could be modelled and distinguished from an unsuccessful one. We specifically asked the following questions:

1. What components of the narrative macrostructures are present, and are the overall macrostructures we have chosen well suited to game stories?

2. Can the source of a story event, as either character-driven or plot-driven, be easily determined, and is it a useful metric for modelling story events?

3. Does this model accurately provide a picture of how events are sequenced together in a typical game story?

4. What is the relationship between the source of story events (as either character-driven or plot-driven) and their ordering, and the quality or success of the story?

5. Are there other factors which affect this relationship, such as the importance of a story event as either a Kernel or Satellite?

Our findings for these questions are discussed in this section: 


\subsubsection{Narrative Macrostructures}

The narrative Macrostructures used in our analysis were Propp's functions, The Hero's Journey, and The Writer's Journey. We found the Writer's Journey to be the best fit for the game stories we analysed. The Hero's Journey was a moderate fit. Propp's functions were not a good macrostructure to model our game stories. We will limit discussion of our findings with regards to Propp's functions in this section and instead focus on the Hero's and Writer's Journeys.

Many similarities were observed between the Hero's Journey and the Writer's Journey. Given that the Writer's Journey is an adaptation of the Hero's Journey, this is not overly surprising. The Writer's Journey is a more streamlined version of the Hero's Journey and cuts out many of the less common structural elements. The two elements that were uncommon from the Writer's Journey, "Refusal of the Call" and "The Road Back", were shared with their equivalents within the Hero's Journey. However, the Hero's Journey contained an additional three elements which were uncommon in our data, which were not shared with the Writer's Journey.

Some interesting insights can be made about the conformance of macrostructures by game stories. "The Road of Trials" from the Hero's Journey, or the Writer's Journey equivalent "Tests, Allies, Enemies" occur the most frequently within all stories. These instances are often where the bulk of the gameplay is concentrated, with different levels and objectives of the game frequently corresponding to this component. 


\subsubsection{Character-Driven and Plot-Driven Events}

We proposed a categorization for story events, identifying them as either characterdriven or plot-driven. This categorization was developed from existing research and theories distinguishing internal and external character goals. We performed a pilot analysis of game stories with our visualizer. This led us to question whether higher quality game stories would feature more character-driven events.

The results of our analysis of character-driven and plot-driven events showed that a game story is mainly powered by character-driven events, but the characters are still at times pitted against external events.

Our qualitative analysis confirmed that lower quality stories featured more plot-driven events and plot-driven events that were less connected to character goals. Character goals were also less often present or identifiable in lower scoring games.

\subsubsection{Event Sequencing}

We found that $95 \%$ of the stories in our data began with a plot-driven event. The probability data we produced for 2-event and 3-event strings provide strong models for how events should be sequenced with respect to character-driven and plot-driven events.

Additionally, we were able to note factors which should limit or override the above probability models. Plot-driven events, for example, are necessary to drive the story forward and provide the characters external obstacles and tasks to overcome along their journey. However, our analysis suggests that these events should be spread out over the course of the 
story, as opposed to clumped together. We observed that games which featured multiple consecutive plot-driven events tended to be lower scoring. This effect was observed beginning with a 3-event string, that is, a P-P-P event string.

We found the Writer's Journey and the Hero's Journey to be useful structures to inform the sequencing of events, with the Writer's Journey being a better fit than the Hero's Journey. We were able to assign probable categorizations of our other variables (Character-Driven / PlotDriven, Kernel / Satellite) to each of these components. If the macrostructure is used to guide the overall arc of the story, these component points can be used to inform generator content along the way. The exception is the "Tests, Allies, Enemies" / "The Road of Trials" component, which is interspersed throughout the story as we have discussed above.

\subsubsection{CD/PD events and story success}

Our results show a clear relationship between the number of plot-driven and characterdriven events in a game with its aggregate critical score. We found that a higher proportion of Character-Driven events was associated with a higher critical score.

It would be tempting to conclude from the above point that the strongest game stories would eventually eliminate plot-driven events altogether in favour of a purely character-driven story. However, it is important to note that even the highest scoring games in our dataset still had roughly $20-30 \%$ plot-driven events. This is in-line with the narrative theories we have discussed which state the necessity for some plot-driven events. 


\subsubsection{Other Factors}

Our finding that a higher percentage of character-driven events led to a higher overall game score held true when applied to just kernel events. That is, if satellites are removed from the analysis, we found that a higher percentage of kernel events being character-driven was also associated with a higher game score.

It is interesting to consider the culture of modern stories, and how that may affect the stories being written for games. Glassner identified that contemporary stories tend to end quickly after the climax, with little to no return arc [1]. Our data is consistent with this observation. In the Hero's Journey, for example, four of the five non-conforming functions occur after the climax and have to do with the return arc.

\subsection{Overall Question}

Our overall question was whether a game story could be modelled structurally based on our chosen categorization factors:

- Character-Driven / Plot-Driven

- Kernel / Satellite

- Main / Chapter / Event level

- Narrative Macrostructures

- The Hero's Journey

○ The Writer's Journey

- Propp's Functions 
We discovered early on with our visualization tool that the Main / Chapter / Event categorization was not useful. However, statistical testing on the other elements allowed us to confirm that the Character-Driven / Plot-Driven, Kernel / Satellite, and Hero's Journey and Writer's Journey Macrostructures were useful towards creating a game story model.

We found that a Plot-Driven event is typically used at the start of the story. We recommend The Writer's Journey to guide the overall progression of the story. "The Refusal of the Call", and "The Road Back" components of this macrostructure may be omitted. Event structure from event to event should be based on the probability values defined in Chapter 4 . Both 2-length and 3-length probabilities should be used to inform the final decision on whether a plot-driven or character-driven event should be used. This decision can and should be overridden by other parts of the model, such as the limitation on P-P-P (and longer) event strings. If an event is decided to be a component of the macrostructure, information about the type of event used for that component should override the probability system. For example, we found "The Ordeal" component of the Writer's Journey to strongly feature character-driven kernel events. Should "The Ordeal" appear in the generated story, this preference for character-driven kernels should override the probability system's decision.

Following these rules will lead to a game story which follows the arc of The Writer's Journey, and which features a higher proportion of Character-Driven events. A higher proportion of Kernel events will also be Character-Driven.

We were able to distinguish between high and low rated stories through some metrics, but not through others. We were able to show a significant difference in the percentage of 
character-driven events between top rated and low rated games. We were also able to observe differences in event sequencing, particularly with strings of plot-driven events. Longer strings of plot-driven events are less frequent in higher scoring games. We were also able to identify significant differences in the probabilities of moving between character-driven and plot-driven events between our highest scoring and lowest scoring games. We were unable to make comparisons between high and low scoring games and their usage and conformance to narrative macrostructures.

\subsection{Weaknesses of Research}

Much of our statistical analysis is based on the assumption that the aggregate critical score of the game is a fair metric for the quality of the story. We are aware that this is an imperfect metric, but it was the fairest and objective metric we could find given the subjective nature of stories. However, given that this is an imperfect metric, our data analysis and conclusions may be less accurate.

One of our stated goals was to contrast higher and lower scoring games in order to draw stronger conclusions about what should or should not be present in a game story. However, our final dataset does not contain enough lower scoring games to allow us to make these distinctions in most cases. While preliminary conclusions can be drawn from splitting the data into halves or thirds, these comments should be taken as preliminary in nature, as the split data sets are not large enough to be entirely valid. In our opinion, the overall dataset is still a valid source to analyse, with a median score of $84.42 \%$ and an average score of $80.64 \%$. 
Data collection is another potential source of error in our methodology. Ideally, data collection would have been done by multiple people, or data collected would have been reviewed and discussed by more than just myself. The definitions of classifications such as Character-Driven versus Plot-Driven, or Kernel versus Satellites are very clear. However, in practice and due to research limitations, classifying story events depended on the researcher's subjective perception of each event. Another person may have classified events differently. However, having a single classifier resulted in consistent classification. Therefore any error within the dataset should be relatively constant across all games.

In our data analysis, a significance level ( $p$-value) of 0.05 was used across the board. Given that sample sizes in some of our analyses vary widely, a single significance value may not have been appropriate for all our tests to indicate significance.

The majority of the studied games were linear. A few of the games featured branching storylines. In these cases, only a single branch was studied. Different branches may have differed in terms of features such as the percentage of events which were character-driven. However, we treated the single studied branch as the sole data structure of the game and attributed it to the aggregate critical score. No hypertext stories were covered in this study.

\subsection{Applications}

The goal of this work was to uncover structural rules within game stories that could be used to assist in game story creation. The model we have created has applications across both game development and academia. 
To begin with, our model can be useful to game story writers across a variety of backgrounds and development environments. The model can be used as both a framework and an evaluation tool. As a framework, it gives recommendations to writers on how to structure their stories, and what types of events should be present in different parts of the narrative. The model can also be used to evaluate a narrative after it has been written. This may help writers who wish to improve upon an existing draft of their game story.

Larger studios with high budget games might benefit from a tool which could allow them to mitigate potential failures early on in the development cycle. By having a model to evaluate stories against, studios could better evaluate if a story is likely to be received favourably or unfavourably. Additionally, such a tool may reduce or eliminate the need for user testing or studies on the game's narrative. This has two benefits: firstly, user studies can carry a significant cost, requiring staff to administer the test, hardware and facilities, as well as any compensation for testers. Reducing the need for these tests presents a potentially significant cost savings to developers. Secondly, despite non-disclosure agreements, bringing external people into the studio carries a risk of information being spread to the public before the developer intends.

We feel our model also has applications in procedural generation of stories. Procedural Generation has the benefits of reducing developer overhead [7], allowing for player customization or control over the content [40], and creating more replayable games [41]. Our story model would allow for increased replayability, and reduce development work. However, our model does not allow for online generation, including player customization or tailoring of 
narratives to their desires. Narratives have been shown to see a reduction of quality when reader choice or opinion is allowed [1][30], which is why we recommend generation of fully embedded stories, without allowing for player choice.

We feel our model would be well suited to implementation into an "engine", which would work with and inform a narrative content generator. Our model does not include other components that are necessary for story generation, such as character modelling systems, or suggestions as to how world-state should be managed. These concepts have been well covered in existing research, as we described in Chapter 2. However, existing systems lacked a structuralist view and did not tend to view their stories from an event-based perspective. If our model were to be integrated into a system with a strong character goal model, it would create a generator which addressed this gap. If this work were to continue, the next step would be to research methods of content generation, in order to determine what functionality would be needed in a system built from our model in order to be capable of interfacing or working with a content generator. A content generator could either be selected from existing works or developed so that it was able to interface with and inform itself from our model. Initially we had hoped to develop or locate a content-generator with which to perform this next step in the work, but we determined it was out of the scope of possibility given the timeline of this project.

The content generator would need to contain:

- A character model. This model would need to store internal goals associated with the character. It would also likely contain other modelling information, such as a personality 
model, and information about the character's current state, personality, and mood at the current time of the story.

- Character actions. The system would need a robust definition of actions or speech which a character would undertake. If the current event was slated to be characterdriven, the character action would be determined based on the current state of the character model. If the event was plot-driven, any action would likely be dictated by the event itself.

- A story world. The story world would contain information about all the environments within the world. It would also contain information about various groups of peoples or cultures if they exist, and their relationships to each other. The system would also store the list of characters relevant to the story. Ideally, the system would be able to generate new characters as needed, based on the larger world and cultural rules defined within it.

Such a generator has its own applications within the commercial gaming industry. An engine or content generator created from our model could be used as a development tool. Offloading narrative generation to a procedural system allows for more developer time to be spent on core gameplay systems and mechanics. This is particularly useful for small development teams, or games with rapid release cycles.

We feel that online multiplayer games, particularly Massively Multiplayer Online (MMO) Games, would benefit from the type of generator our model would facilitate. These games typically involve long-running persistent worlds with highly developed rule systems and are designed to remain interesting to players for long periods of time [52]. Usually, this is 
accomplished through frequent content patches, which can include a narrative component. Because of the continuous content cycle common to these games, developmental time and expense are on-going [16]. Additionally, player consumption of content often outpaces what developers are able to produce. Procedural generation of narrative and instances within these games seems positioned to help satisfy these users through continual access to novel content [16] while reducing development overhead and maintaining the overall balance and story-world of the game [44]. 


\section{Conclusion}

\subsection{Summary of Findings}

We modelled a game story as a sequence of events, with each event having properties which would inform the content of that event. We defined the Character-Driven/Plot-Driven categorization and we were able to observe a difference in this metric between high and low scoring games. This suggests that our categorization is useful for game story analysis/generation. We were also able to confirm that Kernel/Satellite, and The Writer's Journey are useful classifications to game structures. We were able to model the ordering of story events based on these properties and make recommendations as to the structure a generator should aspire to when creating a narrative.

\subsection{Summary of Research Method}

Our overall research question is whether a game story can be modelled structurally. We centered our research around the core concepts of character goals, and the distinction between external and internal goals and events. We defined these as character-driven and plotdriven events. We hypothesized that game stories with higher proportions of character-driven events would perform better. We performed a pilot analysis of game stories through graphical analysis. We developed visualizations which assisted us in confirming that our hypothesis was worth studying further.

We fleshed out our dataset to 20 game stories. Data collection was done by playing or watching a playthrough of the games. Data was stored in spreadsheet form. Every event within the story was given a unique entry in the spreadsheet and classified across our variables. Once 
the dataset was complete, both qualitative and quantitative analysis was performed in order to answer our research questions.

\subsection{Future Research}

Our methodology had some weaknesses that could be rectified in future work. A future study could feature more robust data collection methods, with more people overviewing and approving categorization, particularly for properties that can be interpreted subjectively like character-driven / plot-driven. Additionally, the sample size could be made larger, with a focus on gaining more titles across the spectrum of critical scores. When trying to compare high and low scoring games, not every test that we used gave a statistically valid result, due to small sample sizes. However, our reliance on critical scores is also a potential source of weakness, as the score encompasses other factors of the game not related to the narrative. A future work should spend the time to determine if a more effective measure of story quality can be found.

This study raises several questions that would require further work in order to answer. A large unknown is the effect of the medium of a video game on the story structure. For example, is it possible that some satellite events are added purely for gameplay purposes, rather than to flesh out the narrative? In a video game, it could be desired to find reasons to add additional levels, which may not have appeared in another medium such as a print book or a movie. 


\section{References}

[1] A. Glassner, Interactive Storytelling: Techniques for 21st Century Fiction. 2004.

[2] L. Cron, Wired for Story. Ten Speed Press, 2012.

[3] R. Barthes, "An Introduction to the Structural Analysis of Narrative," New Lit. Hist., vol. 6, no. 2, pp. 237-272, 1975.

[4] J. Campbell, The Hero with a Thousand Faces, vol. 63, no. 247. 1949.

[5] V. Propp, "Morphology of the Folk Tale," Folklore, 1928.

[6] C. Vogler, "A Practical Guide to the Hero With a Thousand Faces," 1985.

[7] W. D'Angelo, "EA: AAA Development in Decline," 2013. [Online]. Available: http://www.vgchartz.com/article/251108/ea-aaa-development-in-decline/. [Accessed: 08-Jul-2017].

[8] C. Morris, "As Video Game Development Costs Rise, So Do Risks," CNBC, 2010. [Online]. Available: https://www.cnbc.com/id/35932496. [Accessed: 04-Jan-2018].

[9] K. Salen and E. Zimmerman, Rules of Play: Game Design Fundamentals, vol. 51, no. 3. 2004.

[10] B. Kybartas and C. Verbrugge, "Analysis of ReGEN as a Graph Rewriting System for Quest Generation," vol. 6, no. 2, pp. 228-242, 2013.

[11] N. Shaker, J. Togelius, and M. J. Nelson, Procedural Content Generation in Games: A Textbook and an Overview of Current Research. 2014.

[12] "Speedtree," 2015. [Online]. Available: http://www.speedtree.com/video-gamedevelopment.php.

[13] N. Shaker and A. Liapis, "Lecture 3: Constructive Generation Methods for Dungeons and Levels," pp. 31-55, 2013.

[14] M. Riedl, "Narrative generation: balancing plot and character," vol. 39, pp. 217-268, 2004.

[15] S. R. Turner, "MINSTREL: A computer model of creativity and storytelling," ProQuest Diss. Theses, p. 841-841, 1993.

[16] E. J. Hastings, R. K. Guha, and K. O. Stanley, "Automatic Content Generation in the Galactic Arms Race Video Game," IEEE Trans. Comput. Intell. Al Games, vol. 1, no. 4, pp. 241-248, 2009.

[17] C. Crawford, On Interactive Storytelling. 2013. 
[18] J. Lebowitz and C. Klug, Interactive Storytelling for Video Games. 2011.

[19] S. Chatman, Story and Discourse: Narrative Structure in Fiction and Film, vol. 34. 1978.

[20] M. A. Picucci, "When Video Games Tell Stories: A Model of Video Game Narrative Architectures," Caracter. Estud. Cult. y Criticos la Esfera Digit., vol. 3, no. 2, 2014.

[21] G. Lucas, Star Wars Episode IV: A New Hope. 1977.

[22] L. Wachowski and A. Wachowski, The Matrix. 1999.

[23] W. F. Brewer and E. H. Lichtenstein, "Stories are to entertain: A structural-affect theory of stories," J. Pragmat., vol. 6, no. 5-6, pp. 473-486, 1982.

[24] R. J. Gerrig and A. B. I. Bernardo, "Readers as problem-solvers in the experience of suspense," Poetics, vol. 22, no. 6, pp. 459-472, 1994.

[25] S. Egenfeldt-Nielsen, J. H. Smith, and S. P. Tosca, Understanding video games: The Essential Introduction, 2nd ed. 2013.

[26] G. Frasca, "Simulation versus Narrative: Introduction to Ludology," Video Game Theory Read., pp. 221-235, 2003.

[27] H. Qin, P.-L. Patrick Rau, and G. Salvendy, "Measuring Player Immersion in the Computer Game Narrative," Int. J. Hum. Comput. Interact., vol. 25, no. 2, pp. 107-133, 2009.

[28] A. Niesz and N. Holland, "Interactive Fiction," Crit. Inq., vol. 11, no. 1, 1984.

[29] E. Aarseth, Cybertext: Perspectives on Ergodic Literature. The Johns Hopkins University Press, 1997.

[30] J. H. Murray, Hamlet on the Holodeck. 1997.

[31] L. Sheldon, Character Development and Storytelling for Games. Course Technology, 2004.

[32] J. D. Bolter and M. Joyce, "Hypertext and creative writing," Proceeding ACM Conf. Hypertext - HYPERTEXT'87, no. November, pp. 41-50, 1987.

[33] N. Montfort, "Fretting the player character," in Second person: Role-playing and story in games and playable media, 2007, pp. 139-146.

[34] S. Chauvin, G. Levieux, J. Y. Donnart, and S. Natkin, "Making sense of emergent narratives: An architecture supporting player-Triggered narrative processes," 2015 IEEE Conf. Comput. Intell. Games, CIG 2015 - Proc., pp. 91-98, 2015.

[35] J. Togelius, E. Kastbjerg, D. Schedl, and G. N. Yannakakis, "What is Procedural Content Generation ? Mario on the borderline," Proc. 2nd Int. Work. Proced. Content Gener. Games, p. 6, 2011. 
[36] J. Liao, J. Yu, and L. Jia, "Procedural modeling of water caustics and foamy water for cartoon animation," Proc. - Pacific Conf. Comput. Graph. Appl., vol. 12, no. 60933007, pp. 1-4, 2010.

[37] J. R. Meehan, "The metanovel: writing stories by computer," Yale University, 1976.

[38] Mojang, "Minecraft." 2011.

[39] Hello Games, “No Man's Sky.” 2016.

[40] G. N. Yannakakis and J. Togelius, "Experience-driven procedural content generation," IEEE Trans. Affect. Comput., vol. 2, no. 3, pp. 147-161, 2011.

[41] G. Smith, "Understanding Procedural Content Generation: a Design-Centric Analysis of the Role of PCG in Games," Proc. 2014 SIGCHI Conf. Hum. Factors Comput. Syst. (CHI 2014), pp. 917-926, 2014.

[42] P. Gervás, "Computational Approaches to Storytelling and Creativity," Al Mag., vol. 30, p. 49, 2009.

[43] H. Barber, "Generator of Adaptive Dilemma-based Interactive Narratives," IEEE Trans. Comput. Intell. Al Games, vol. 1, no. 4, pp. 1-18, 2009.

[44] K. E. Merrick, A. Isaacs, M. Barlow, and N. Gu, "A shape grammar approach to computational creativity and procedural content generation in massively multiplayer online role playing games," Entertain. Comput., vol. 4, no. 2, pp. 115-130, 2013.

[45] B. C. Bae and R. M. Young, "A computational model of narrative generation for surprise arousal," IEEE Trans. Comput. Intell. Al Games, vol. 6, no. 2, pp. 131-143, 2014.

[46] D. Thue, V. Bulitko, M. Spetch, and E. Wasylishen, "Interactive Storytelling: A Player Modelling Approach," Proc. Third Artif. Intell. Interact. Digit. Entertain. Conf., vol. Associatio, no. July, pp. 43-48, 2007.

[47] H. M. Chang and V. W. Soo, "Planning-based narrative generation in simulated game universes," IEEE Trans. Comput. Intell. Al Games, vol. 1, no. 3, pp. 200-213, 2009.

[48] N. Dehn, "Story Generation After TALE-SPIN," Proc. 7th Int. Jt. Conf. Artif. Intell. (IJCAI '81), pp. 16-18, 1981.

[49] M. Theune, S. Faas, A. Nijholt, and D. Heylen, "The Virtual Storyteller," Proc. Work. Storytell. Collab. Virtual Environ., vol. 23, no. 2, pp. 20-21, 2002.

[50] E. Aarseth, "From Hunt the Wumpus to EveQuest: Introduction to Quest Theory," Entertain. Comput., pp. 496-506, 2005.

[51] Google, "Google Visualization API Reference," 2017. [Online]. Available: https://developers.google.com/chart/interactive/docs/reference. [Accessed: 11-Nov- 
2017].

[52] N. Ducheneaut, "Building an MMO With Mass Appeal: A Look at Gameplay in World of Warcraft," Games Cult., vol. 1, no. 4, pp. 281-317, 2006. 


\section{Appendix 1: Summary of Propp's Functions}

\begin{tabular}{|c|c|c|}
\hline Function & Shorthand & \\
\hline One of the members of a family absents himself from home & Absentation & $\beta$ \\
\hline An interdiction is addressed to the hero & Interdiction & $\gamma$ \\
\hline The interdiction is violated & Violation & $\delta$ \\
\hline The villain makes an attempt at reconnaissance & Reconnaissance & $\varepsilon$ \\
\hline The villain receives information about his victim & Delivery & $\zeta$ \\
\hline $\begin{array}{l}\text { The villain attempts to deceive his victim in order to take possession } \\
\text { of him or of his belongings }\end{array}$ & Trickery & $\eta$ \\
\hline $\begin{array}{l}\text { The victim submits to deception and thereby unwittingly helps his } \\
\text { enemy }\end{array}$ & Complicity & $\theta$ \\
\hline The villain causes harm or injury to a member of the family & Villainy & A \\
\hline $\begin{array}{l}\text { One member of a family either lacks something or desires to have } \\
\text { something }\end{array}$ & Lack & a \\
\hline $\begin{array}{l}\text { Misfortune or lack is made known; the hero is approached with a } \\
\text { request or command; he is allowed to go or he is dispatched }\end{array}$ & $\begin{array}{l}\text { Mediation, The } \\
\text { connective incident }\end{array}$ & B \\
\hline The seeker agrees to or decides upon counteraction & $\begin{array}{l}\text { Beginning } \\
\text { Counteraction }\end{array}$ & C \\
\hline The hero leaves home & Departure & $\uparrow$ \\
\hline $\begin{array}{l}\text { The hero is tested, interrogated, attacked, etc., which prepares the } \\
\text { way for his receiving either a magical agent or helper }\end{array}$ & $\begin{array}{l}\text { The First Function of } \\
\text { the Donor }\end{array}$ & $\mathrm{D}$ \\
\hline The hero reacts to the actions of the future donor & The Hero's Reaction & $\mathrm{E}$ \\
\hline The hero acquires the use of a magical agent & $\begin{array}{l}\text { Provision / Receipt of } \\
\text { a Magical Agent }\end{array}$ & $\mathrm{F}$ \\
\hline $\begin{array}{l}\text { The hero is transferred, delivered, or led to the whereabouts of an } \\
\text { object of search }\end{array}$ & $\begin{array}{l}\text { Spatial Transference, } \\
\text { Guidance }\end{array}$ & G \\
\hline The hero and the villain join in direct combat & Struggle & $\mathrm{H}$ \\
\hline The hero is branded & Branded & $\mathrm{J}$ \\
\hline The villain is defeated & Victory & $\mathrm{I}$ \\
\hline The initial misfortune or lack is liquidated & $\begin{array}{l}\text { Initial Lack is } \\
\text { Liquidated }\end{array}$ & $\mathrm{K}$ \\
\hline The hero returns & Return & $\downarrow$ \\
\hline The hero is pursued & Pursuit, Chase & $\operatorname{Pr}$ \\
\hline Rescue of the hero from pursuit & Rescue & Rs \\
\hline The hero, unrecognized, arrives home or in another country & Unrecognized Arrival & 0 \\
\hline A false hero presents unfounded claims & Unfounded Claims & $\mathrm{L}$ \\
\hline A difficult task is proposed to the hero & Difficult Task & M \\
\hline The task is resolved & Solution & $\mathrm{N}$ \\
\hline The hero is recognized & Recognition & Q \\
\hline The false hero or villain is exposed & Exposure & Ex \\
\hline The hero is given a new appearance & Transfiguration & $\mathrm{T}$ \\
\hline The villain is punished & Punishment & $\mathrm{U}$ \\
\hline The hero is married and ascends the throne & Wedding & W \\
\hline
\end{tabular}




\section{Appendix 2: Narrative Macrostructure Equivalency Comparison}

\begin{tabular}{|c|c|c|c|}
\hline Propp & & Campbell & Vogler \\
\hline Absentation & $\beta$ & & \\
\hline Interdiction & $\gamma$ & & \\
\hline Violation & $\delta$ & & \\
\hline Reconnaissance & $\varepsilon$ & & \\
\hline Delivery & $\zeta$ & & \\
\hline Trickery & $\eta$ & & \\
\hline Complicity & $\theta$ & & \\
\hline Villainy & A & & \\
\hline Lack & $\mathrm{a}$ & & Ordinary World \\
\hline $\begin{array}{l}\text { Mediation, The } \\
\text { connective incident }\end{array}$ & B & The Call to Adventure & Call to Adventure \\
\hline Beginning Counteraction & $\mathrm{C}$ & The Refusal of the Call? & Refusal of the Call? \\
\hline Departure & $\uparrow$ & The Crossing of the First Threshold & Crossing the First Threshold \\
\hline $\begin{array}{l}\text { The First Function of the } \\
\text { Donor }\end{array}$ & $\mathrm{D}$ & $\begin{array}{l}\text { The Meeting with the Goddess / } \\
\text { Woman as Temptress / Road of } \\
\text { Trials? }\end{array}$ & Meeting with the Mentor \\
\hline The Hero's Reaction & $\mathrm{E}$ & & \\
\hline $\begin{array}{l}\text { Provision / Receipt of a } \\
\text { Magical Agent }\end{array}$ & $\mathrm{F}$ & Supernatural Aid & Tests, Allies, Enemies \\
\hline $\begin{array}{l}\text { Spatial Transference, } \\
\text { Guidance }\end{array}$ & G & The Road of Trials & Tests, Allies, Enemies \\
\hline Struggle & $\mathrm{H}$ & Apotheosis & Approach to the Inmost Cave? \\
\hline Branded & $\mathrm{J}$ & & \\
\hline Victory & 1 & Atonement with the Father & Reward \\
\hline Initial Lack is Liquidated & $\mathrm{K}$ & The Ultimate Boon & \\
\hline Return & $\downarrow$ & & The Road Back \\
\hline Pursuit, Chase & $\mathrm{Pr}$ & The Magic Flight & \\
\hline Rescue & Rs & Rescue from Without & \\
\hline Unrecognized Arrival & $\mathrm{o}$ & & \\
\hline Unfounded Claims & $\mathrm{L}$ & & \\
\hline Difficult Task & $\mathrm{M}$ & & \\
\hline Solution & $\mathrm{N}$ & & \\
\hline Recognition & $Q$ & & \\
\hline Exposure & Ex & & \\
\hline Transfiguration & $T$ & Master of the Two Worlds & The Resurrection \\
\hline Punishment & $U$ & & \\
\hline Wedding & $\mathrm{W}$ & Freedom to Live & Return with the Elixir \\
\hline
\end{tabular}




\section{Appendix 3: Story Visualizations}

Visualizations are listed from lowest scoring to highest scoring, following the same order as Table 2 in Chapter 4 . Scores for games are not listed here.

Arc

Dark:

K

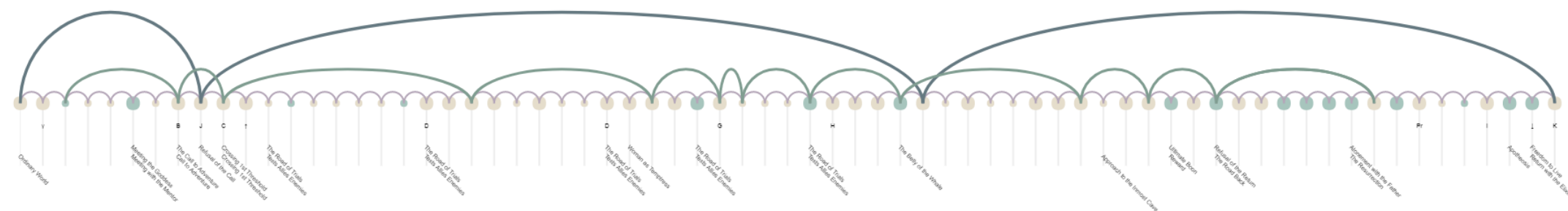

Legendary:

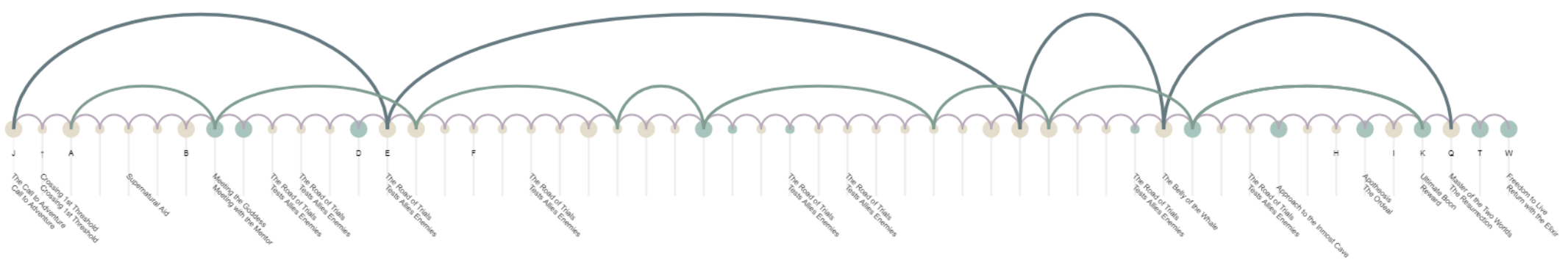


Velvet Assassin:

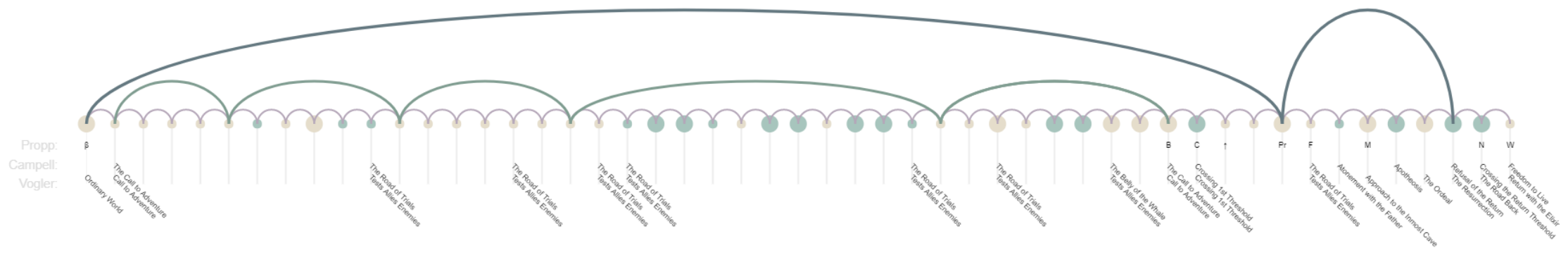

Destiny:

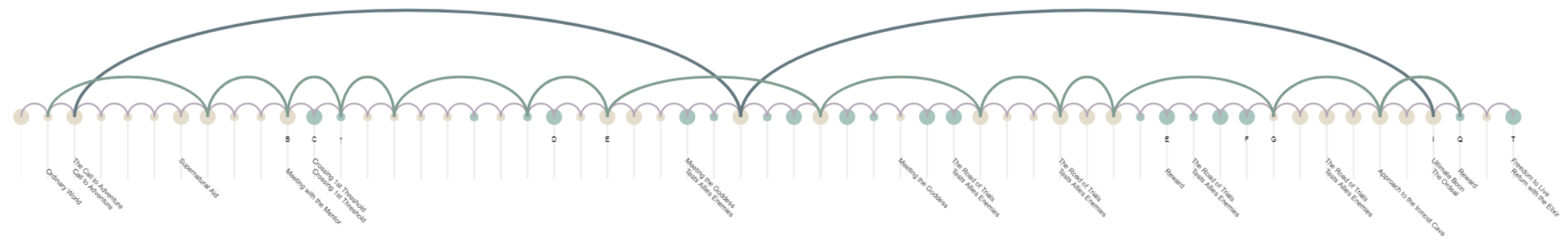

Gravity Rush:

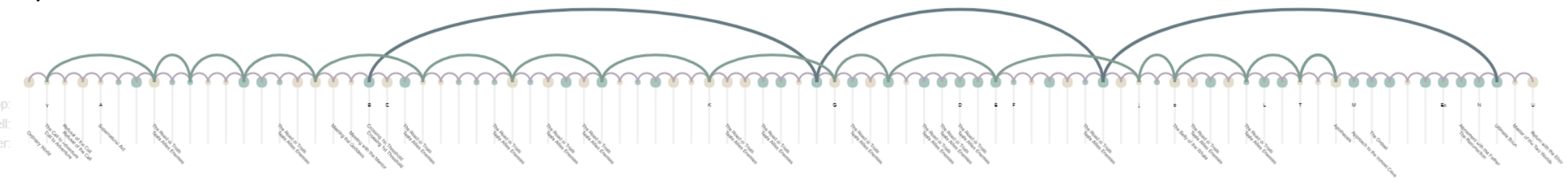

\section{Etrian Odyssey 2 Untold:}

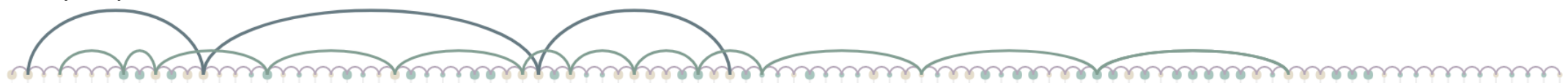
Propp

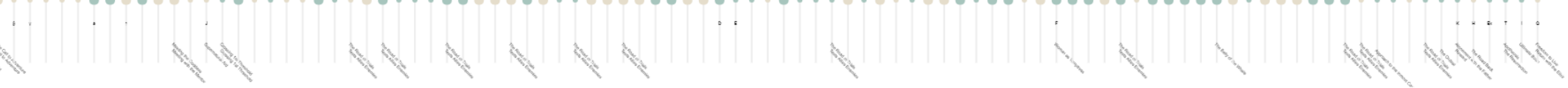


To The Moon:

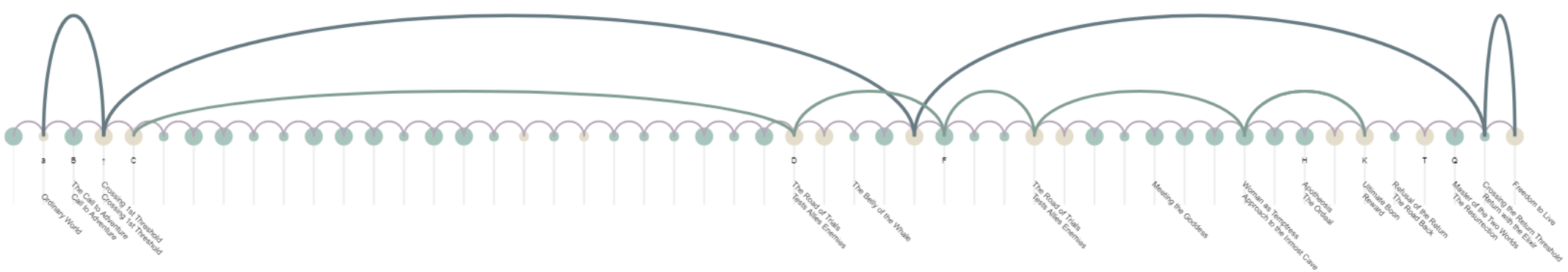

Catherine:

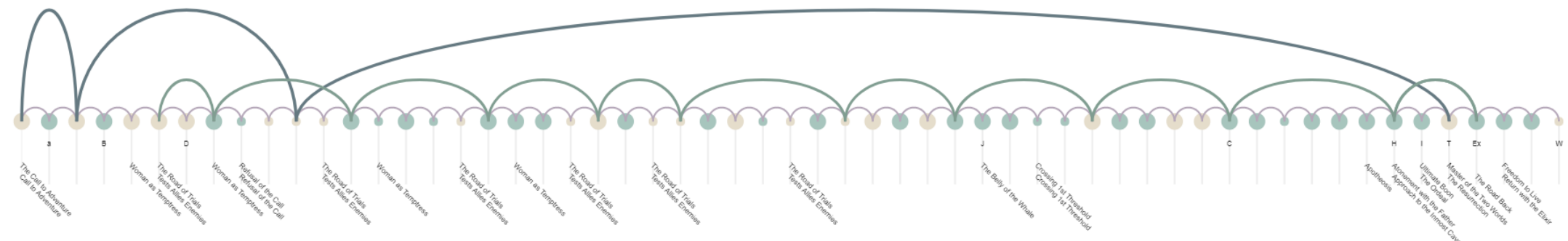

Fire Emblem Fates: Conquest:

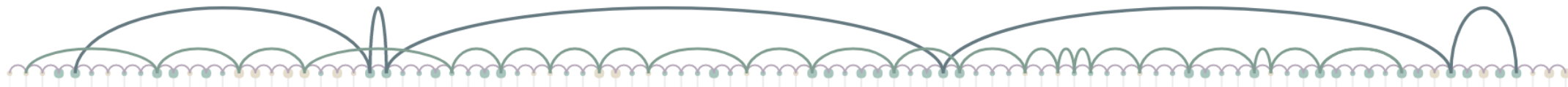

Uncharted: Drake's Fortune:

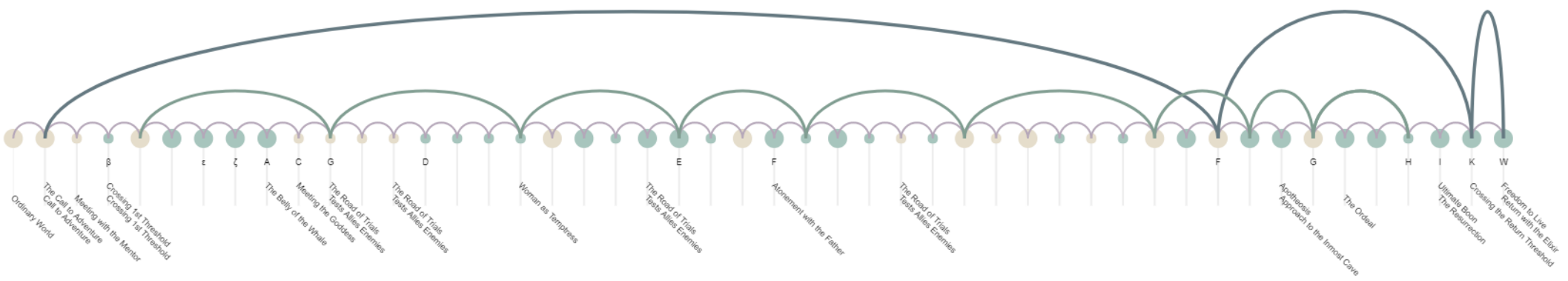




\section{Shadow of Mordor:}

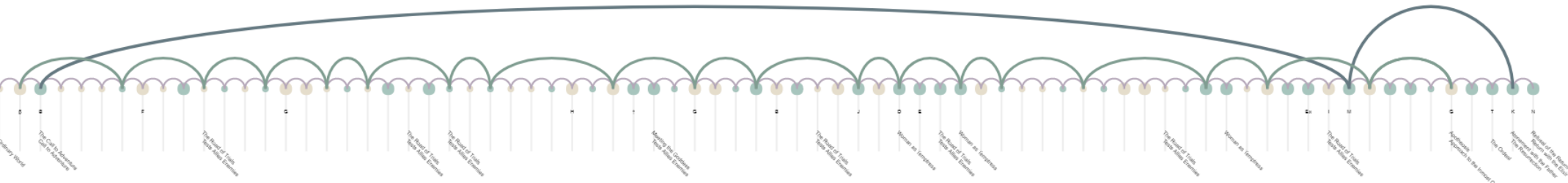

Fire Emblem Fates: Revelations:

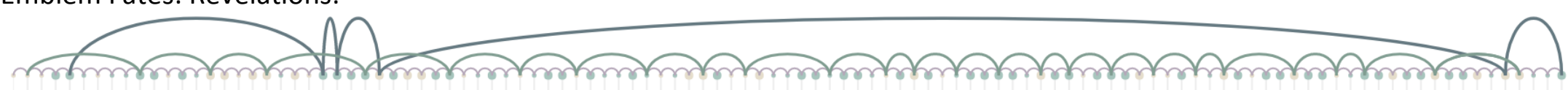

Fire Emblem Fates: Birthright:
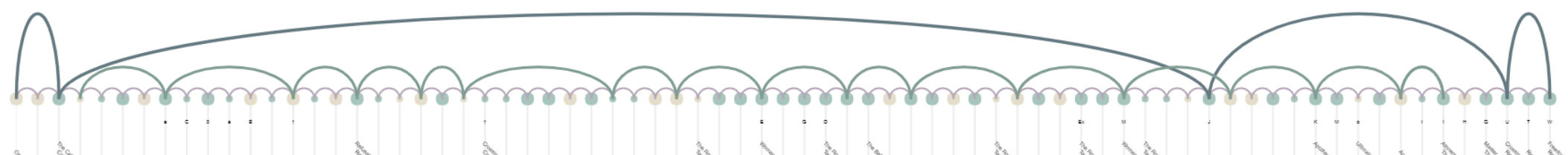

Final Fantasy $\mathrm{X}$ :

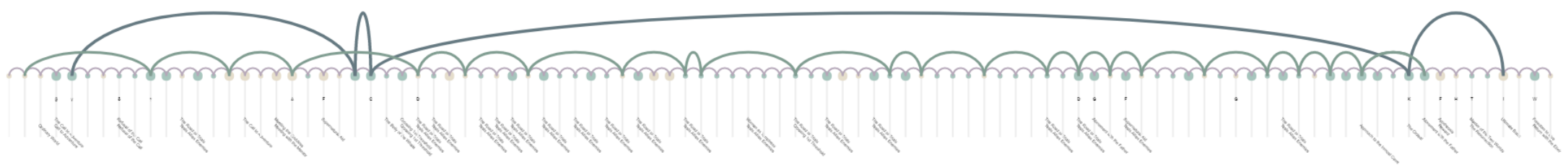

Tactics Ogre: Let Us Cling Together:

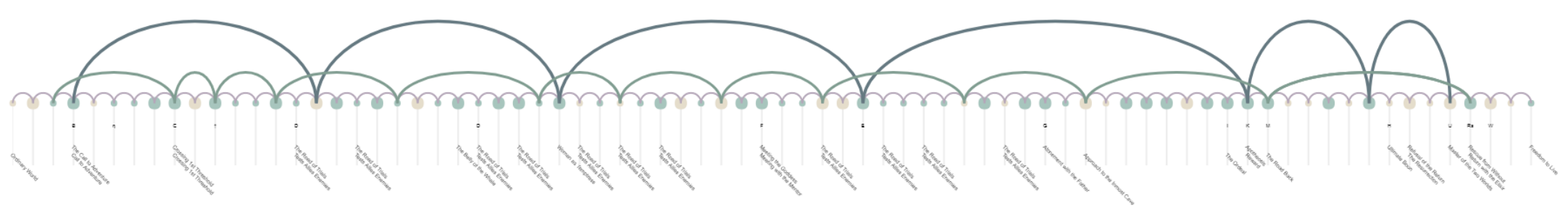




\section{Golden Sun:}

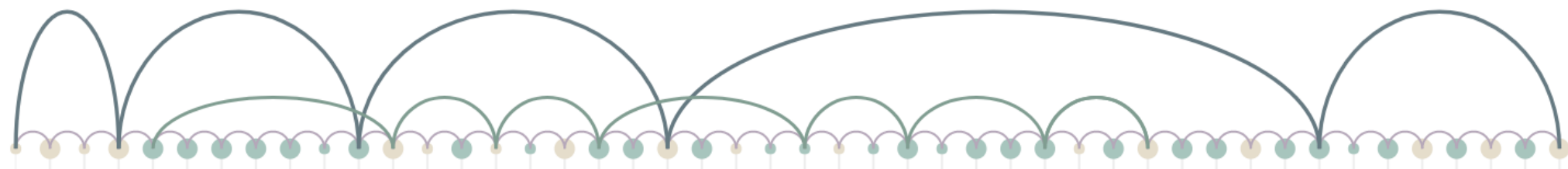

Proply
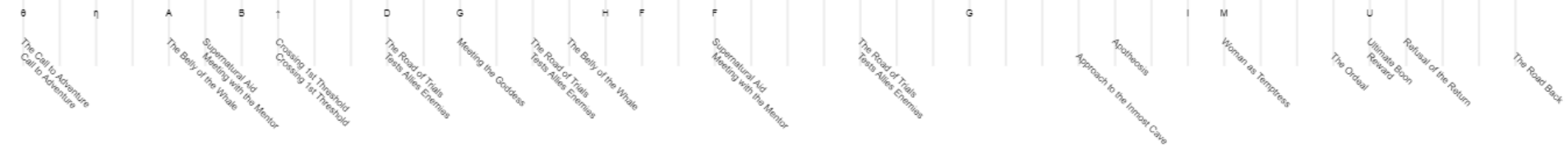

Batman: Arkham Asylum
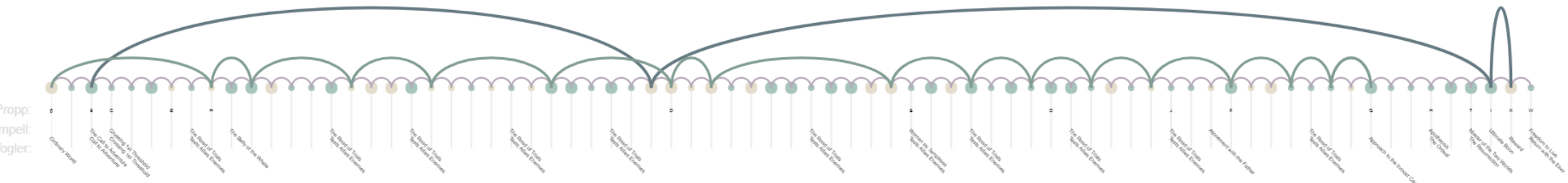

The Last of Us:

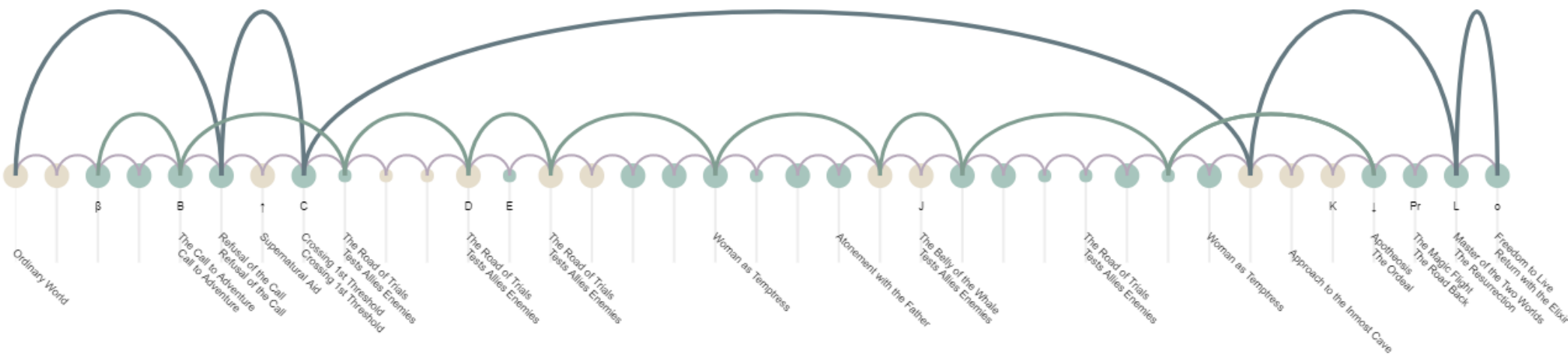


Metal Gear Solid 3:

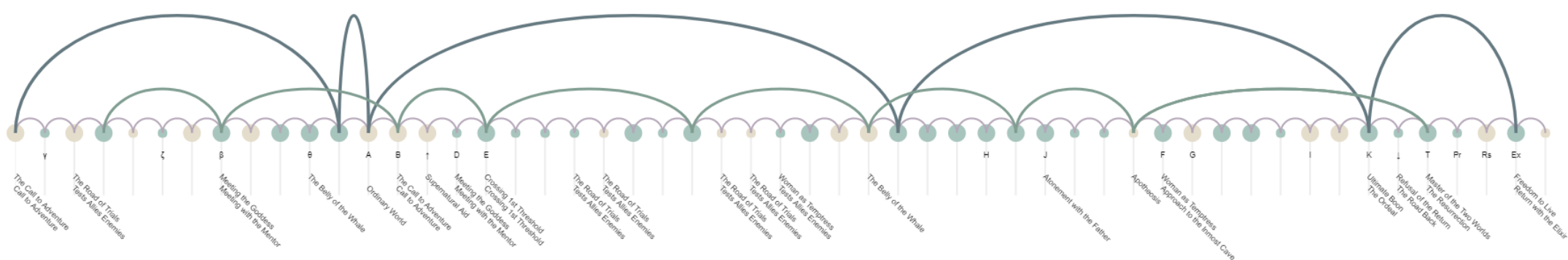

The Legend of Zelda: Ocarina of Time:

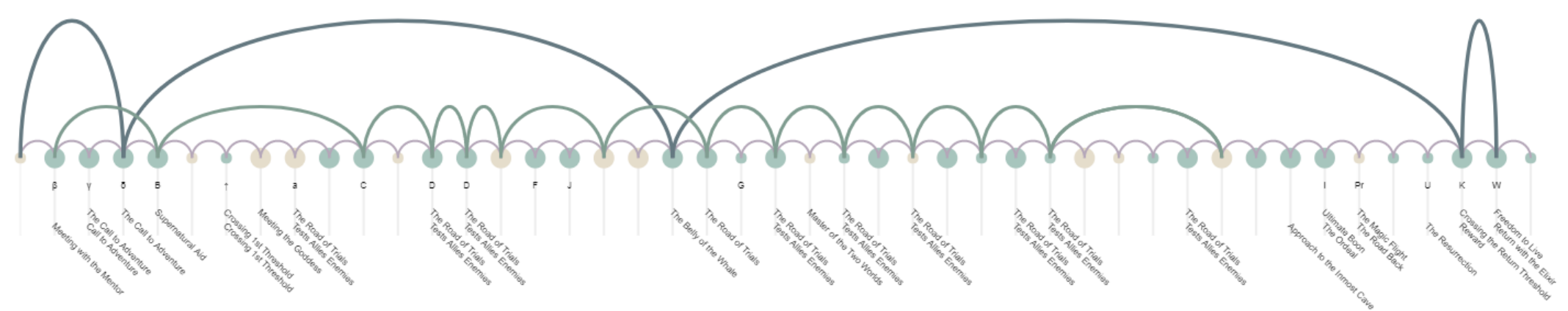




\section{Character Interactions}

Dark:

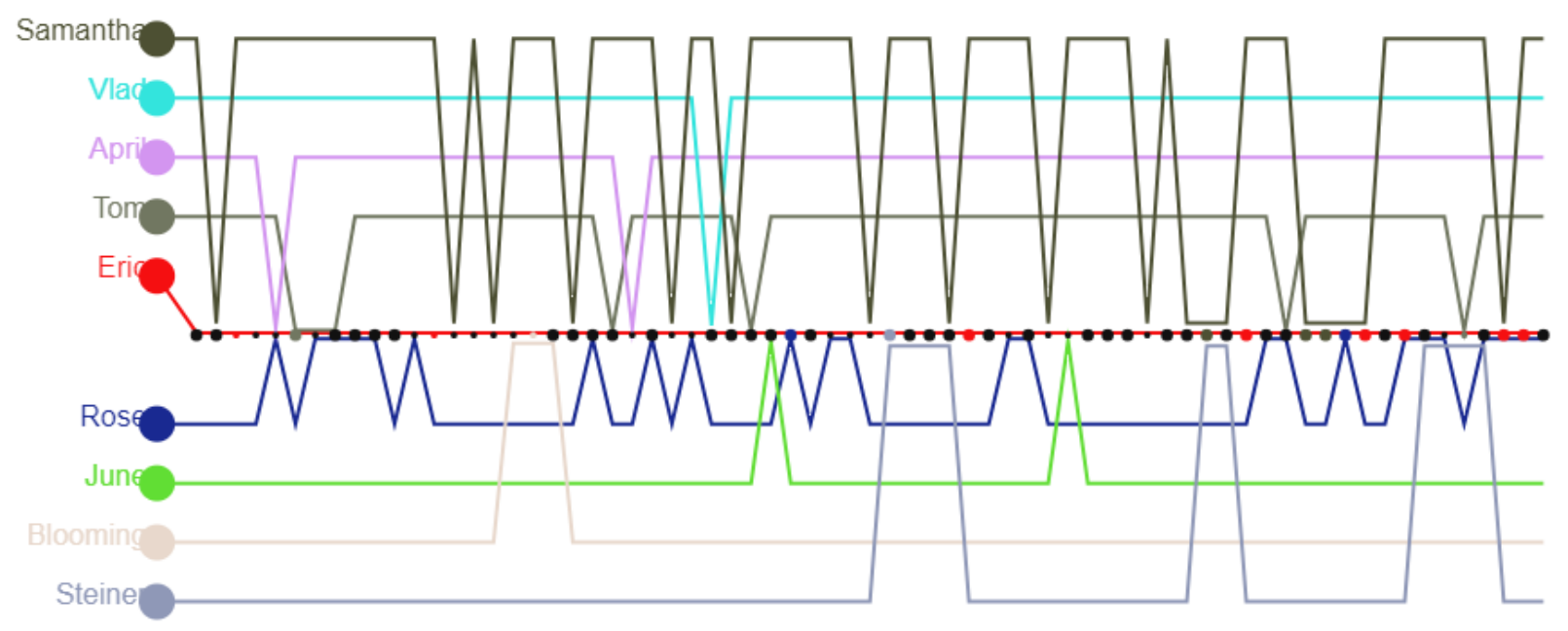

Legendary:

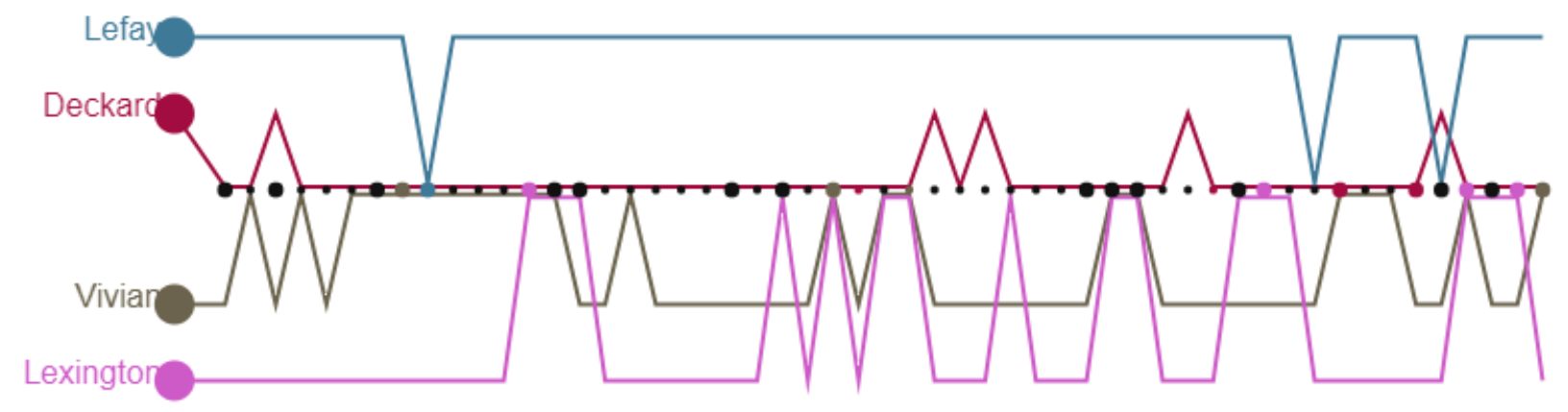

Velvet Assassin:

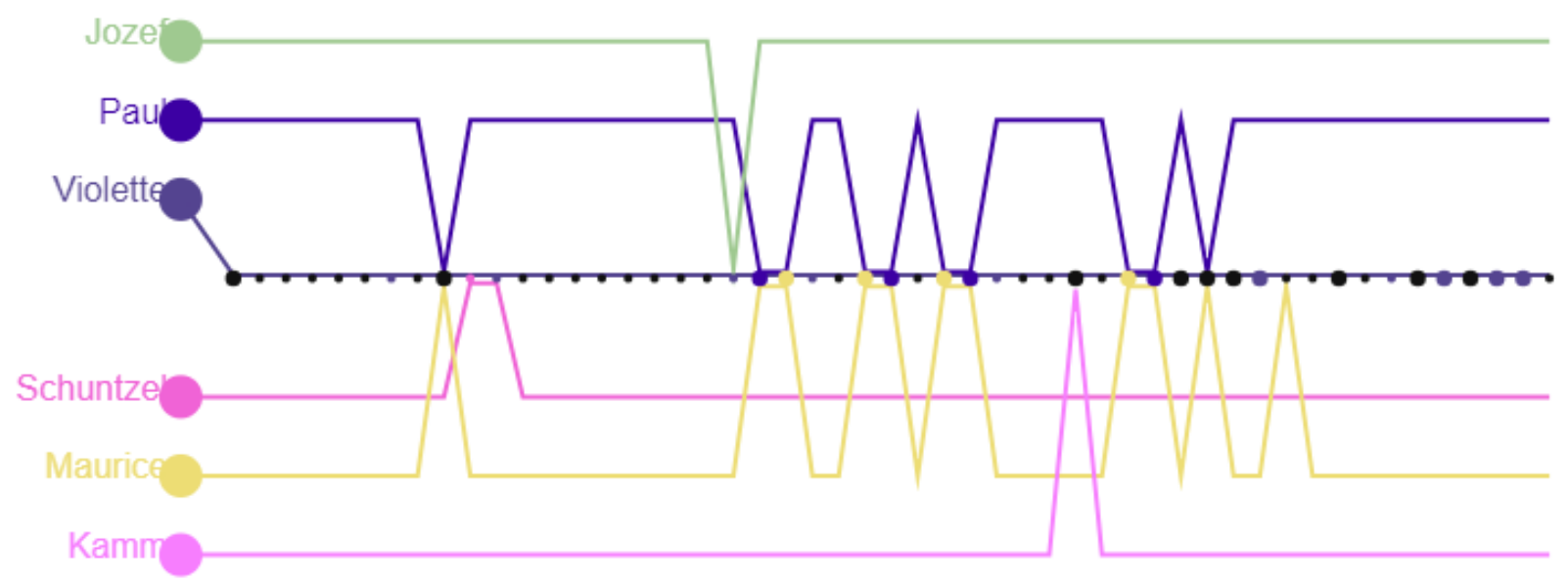


Destiny:

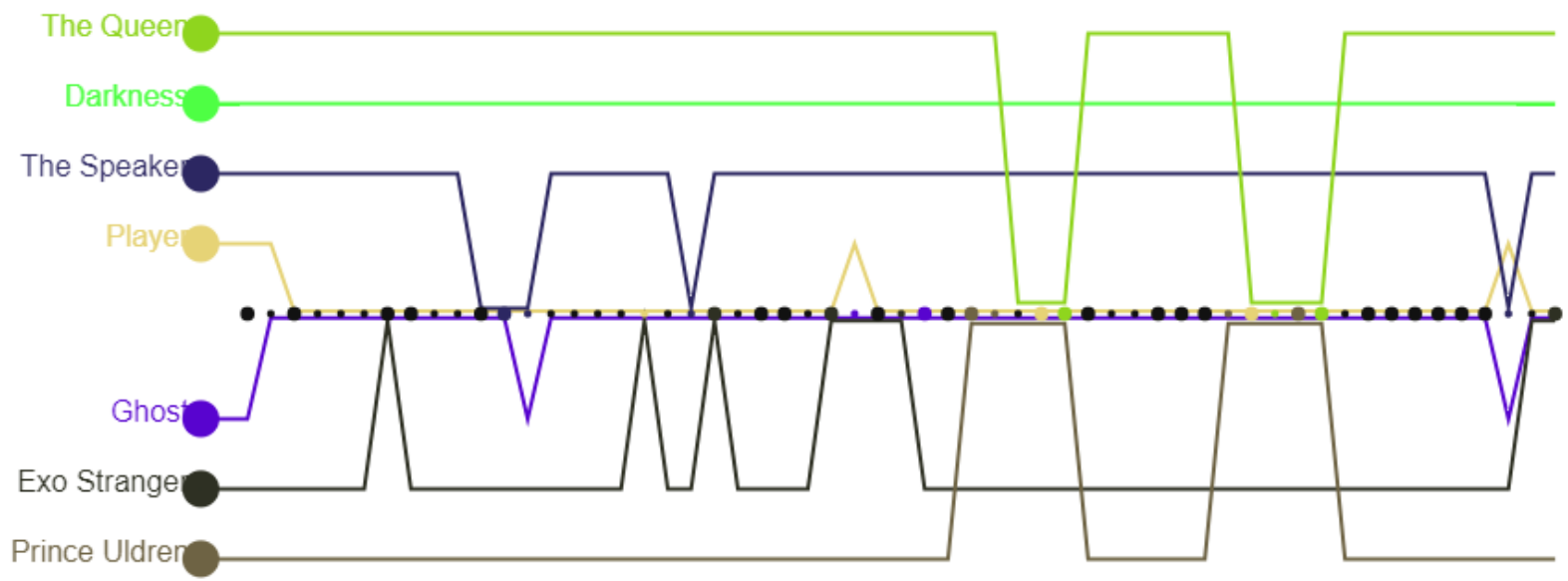

Gravity Rush:

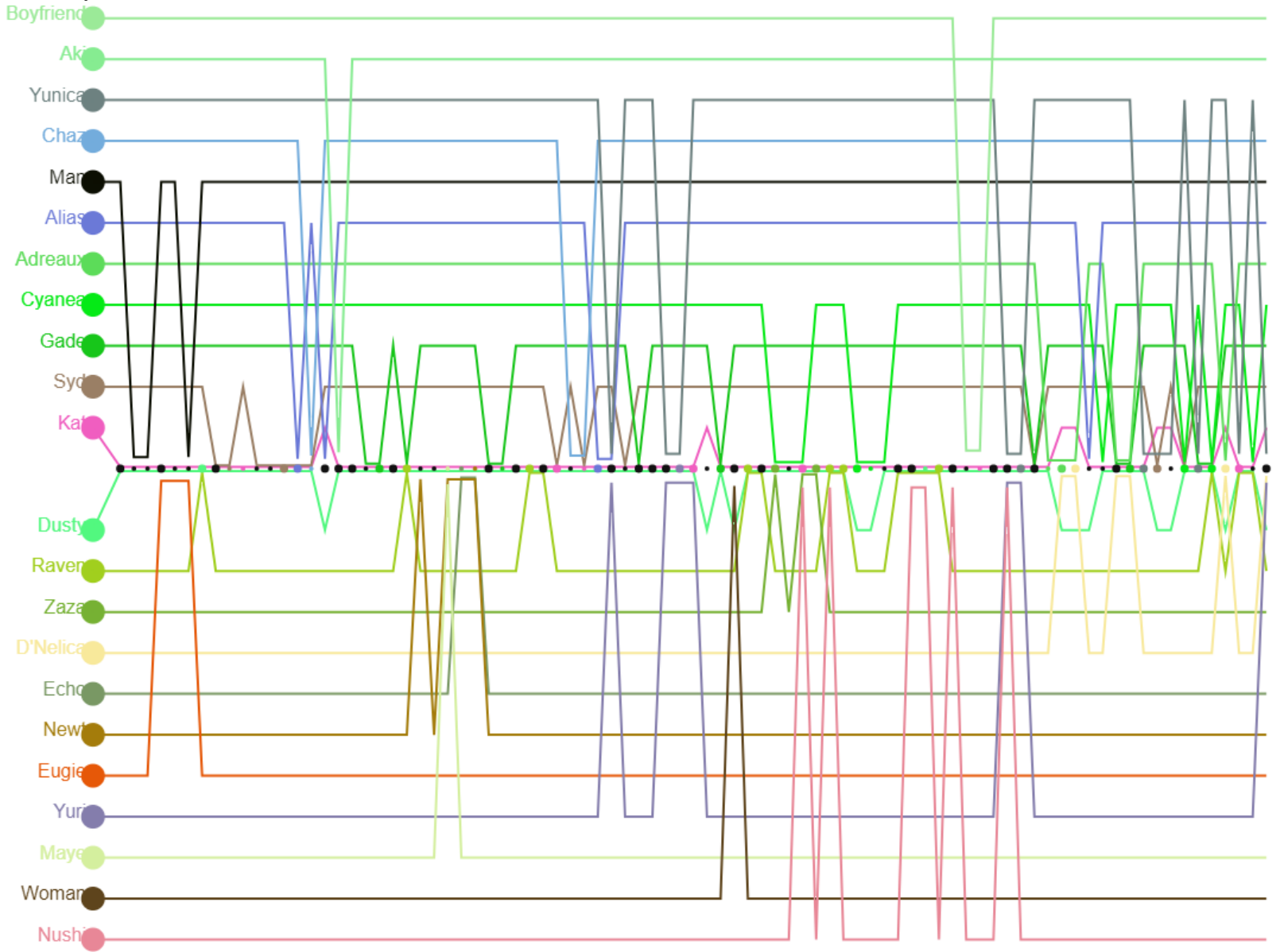


Etrian Odyssey 2 Untold:

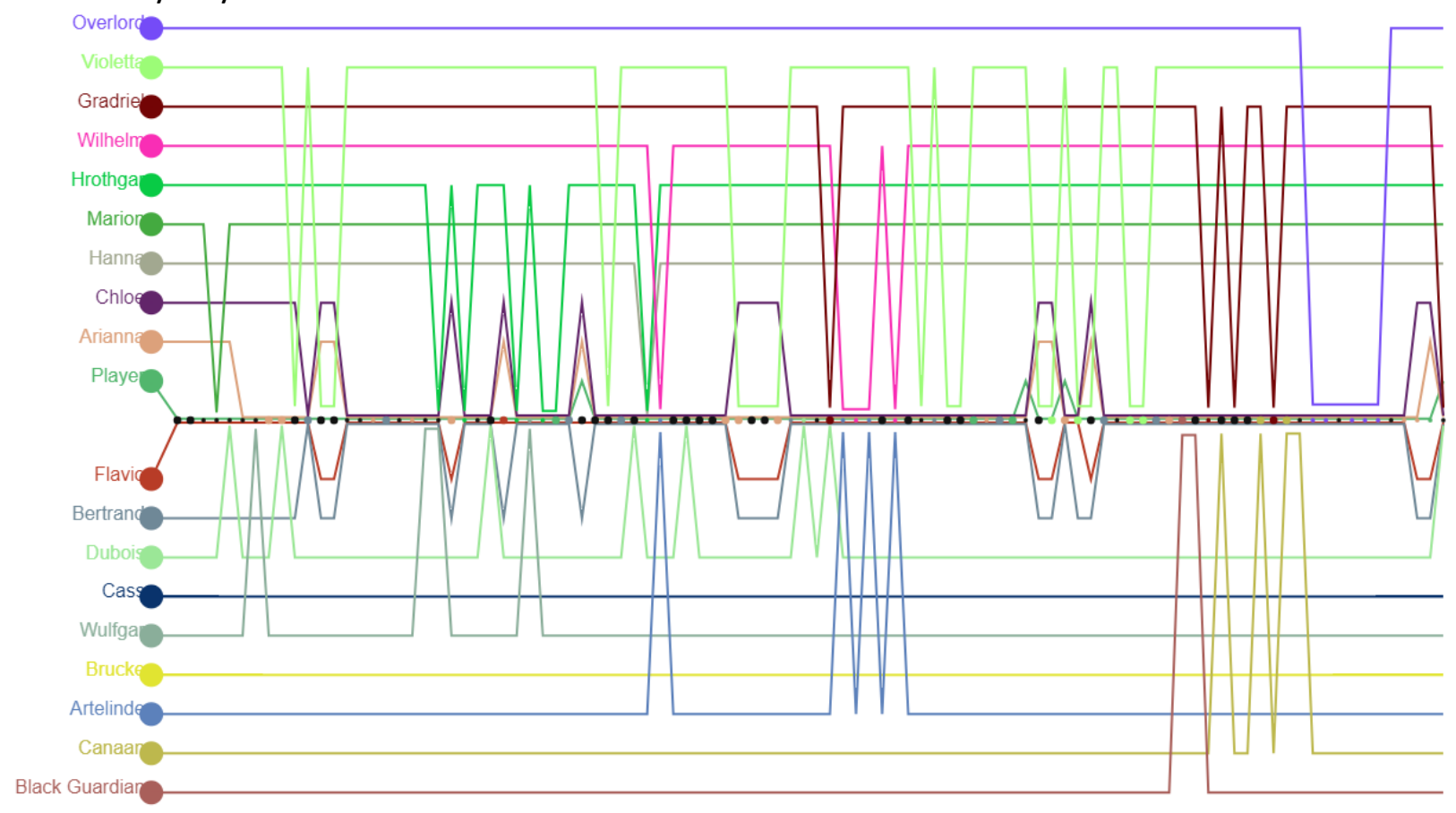

To The Moon:

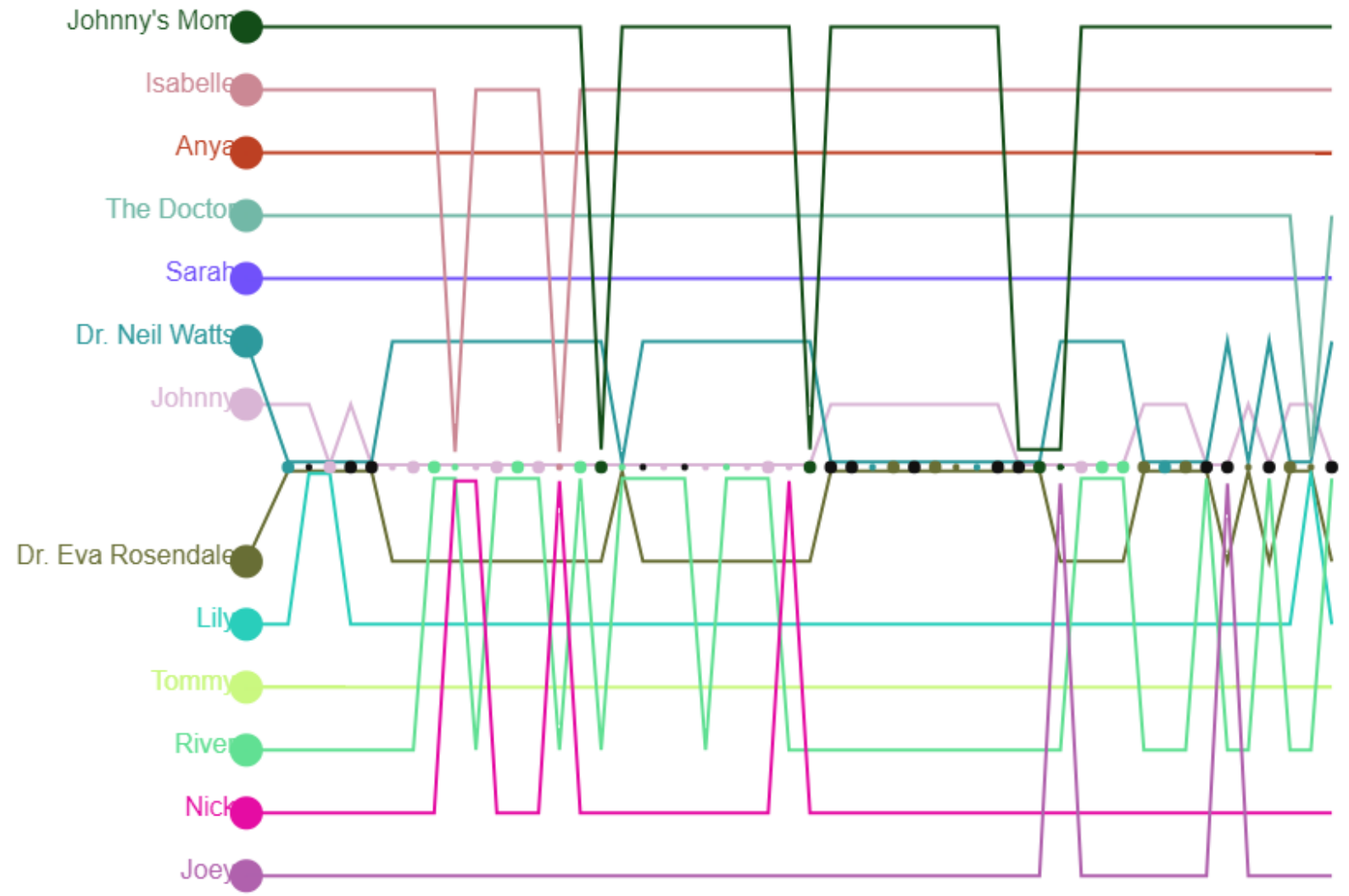


Catherine:

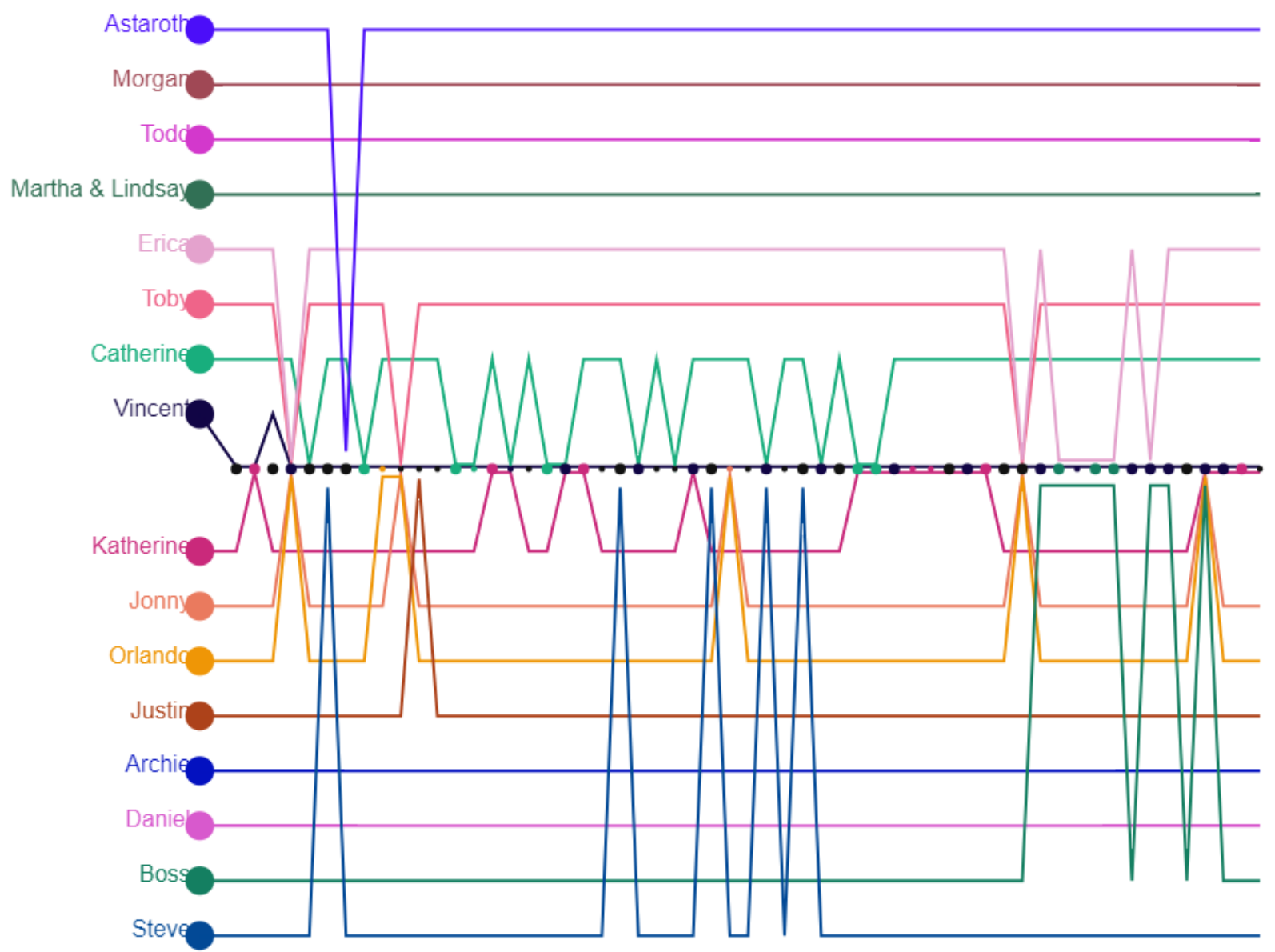


Fire Emblem Fates: Conquest:

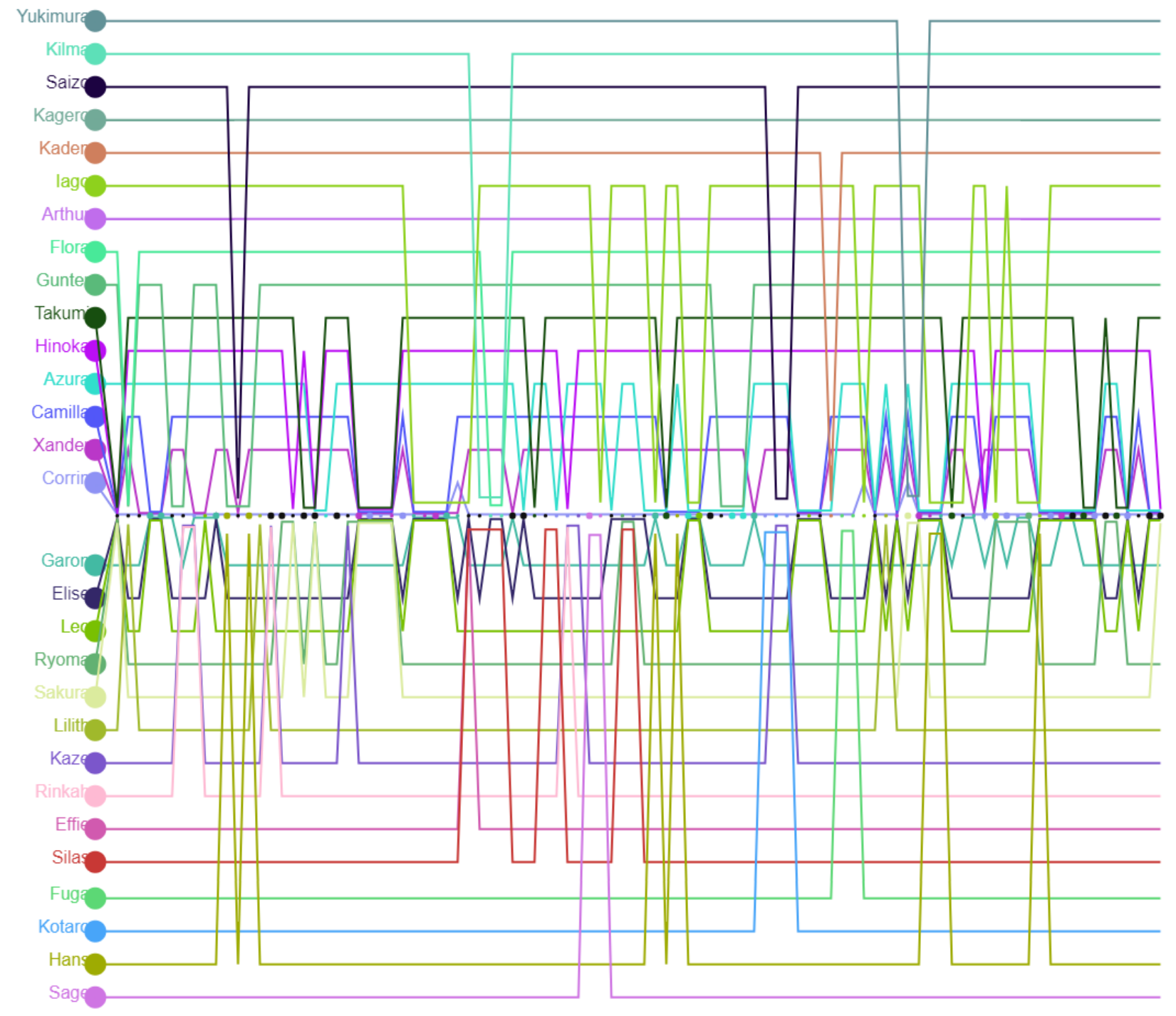


Uncharted: Drake's Fortune:

Sir Francis Drake

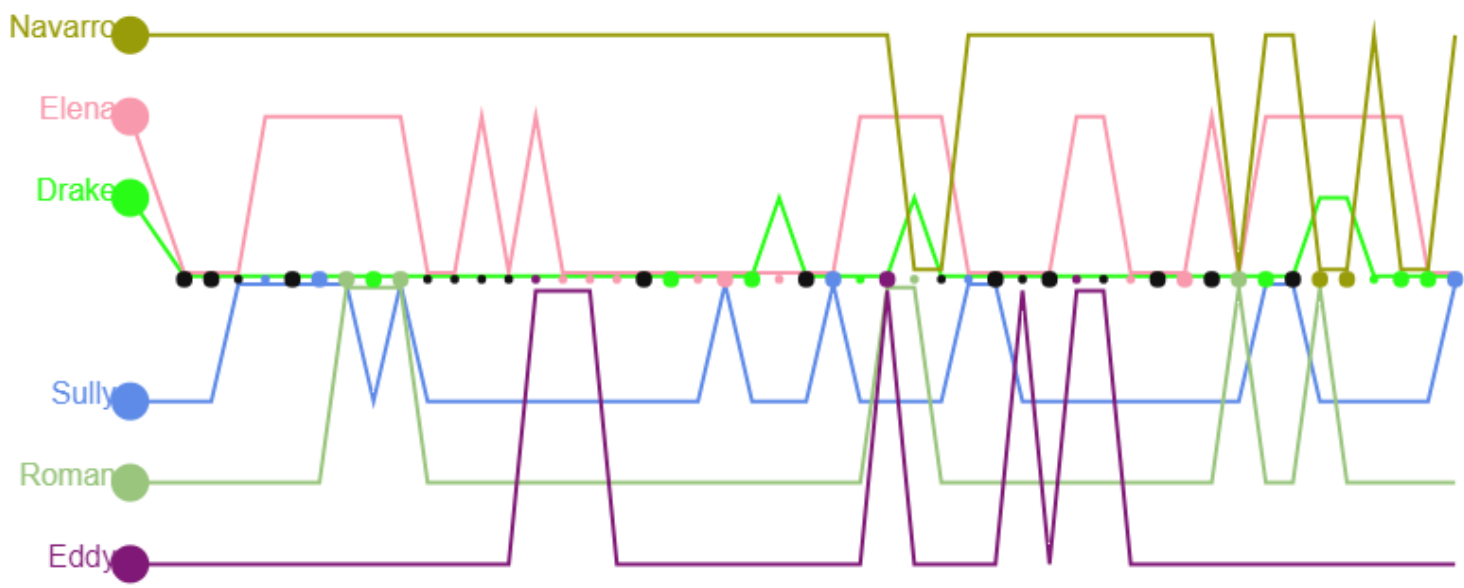

Shadow of Mordor:

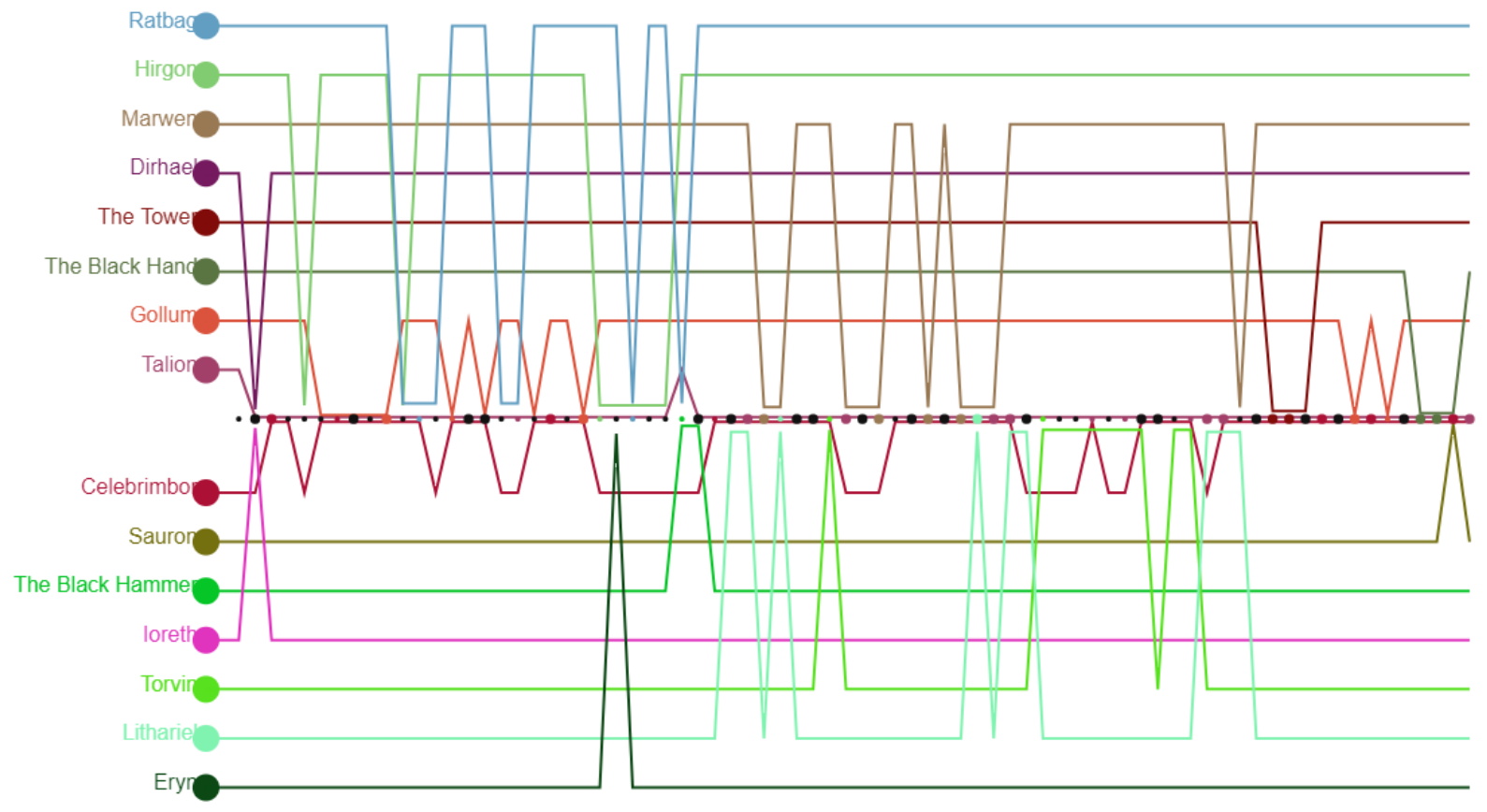


Fire Emblem Fates: Revelations:

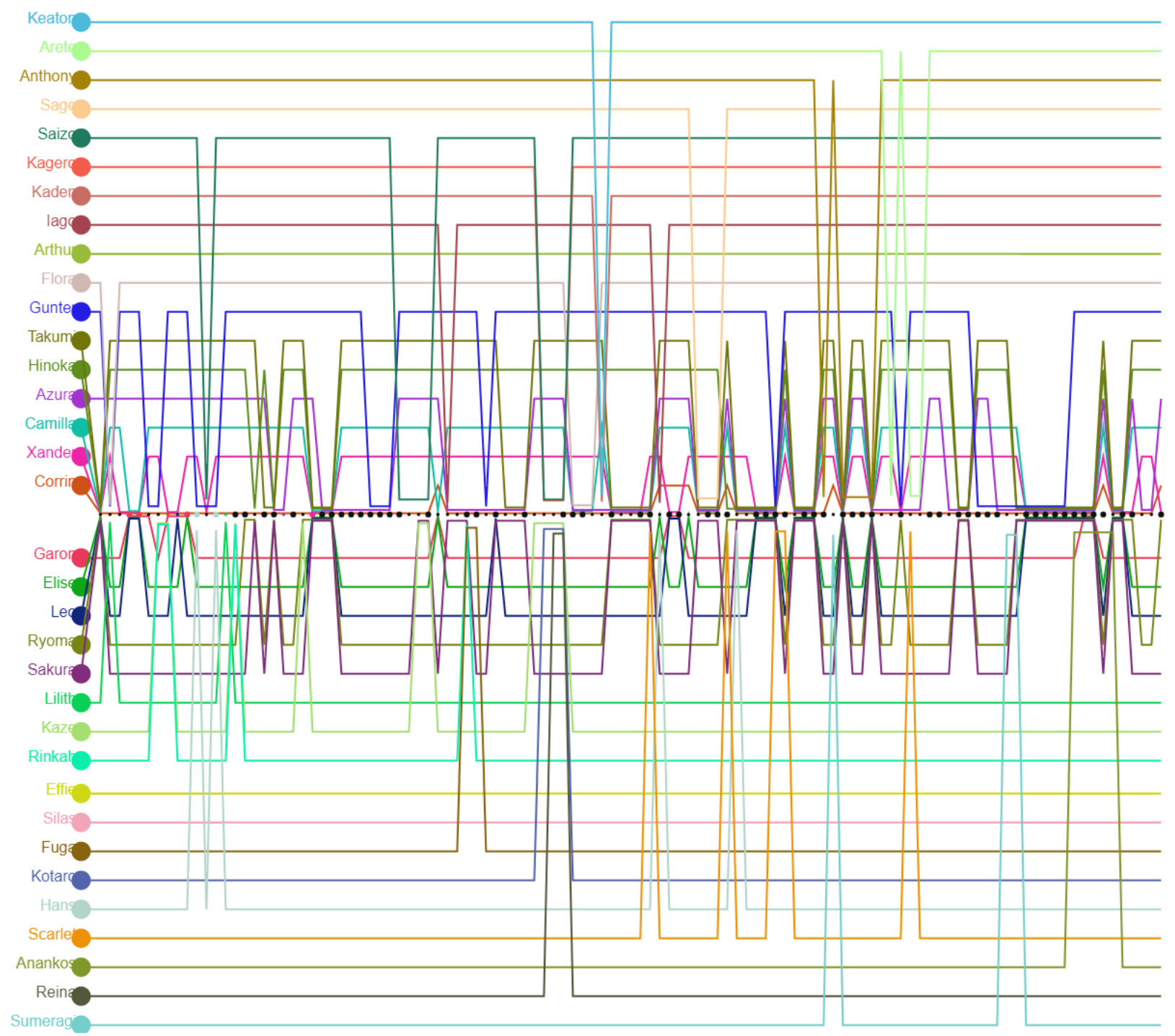


Fire Emblem Fates: Birthright:

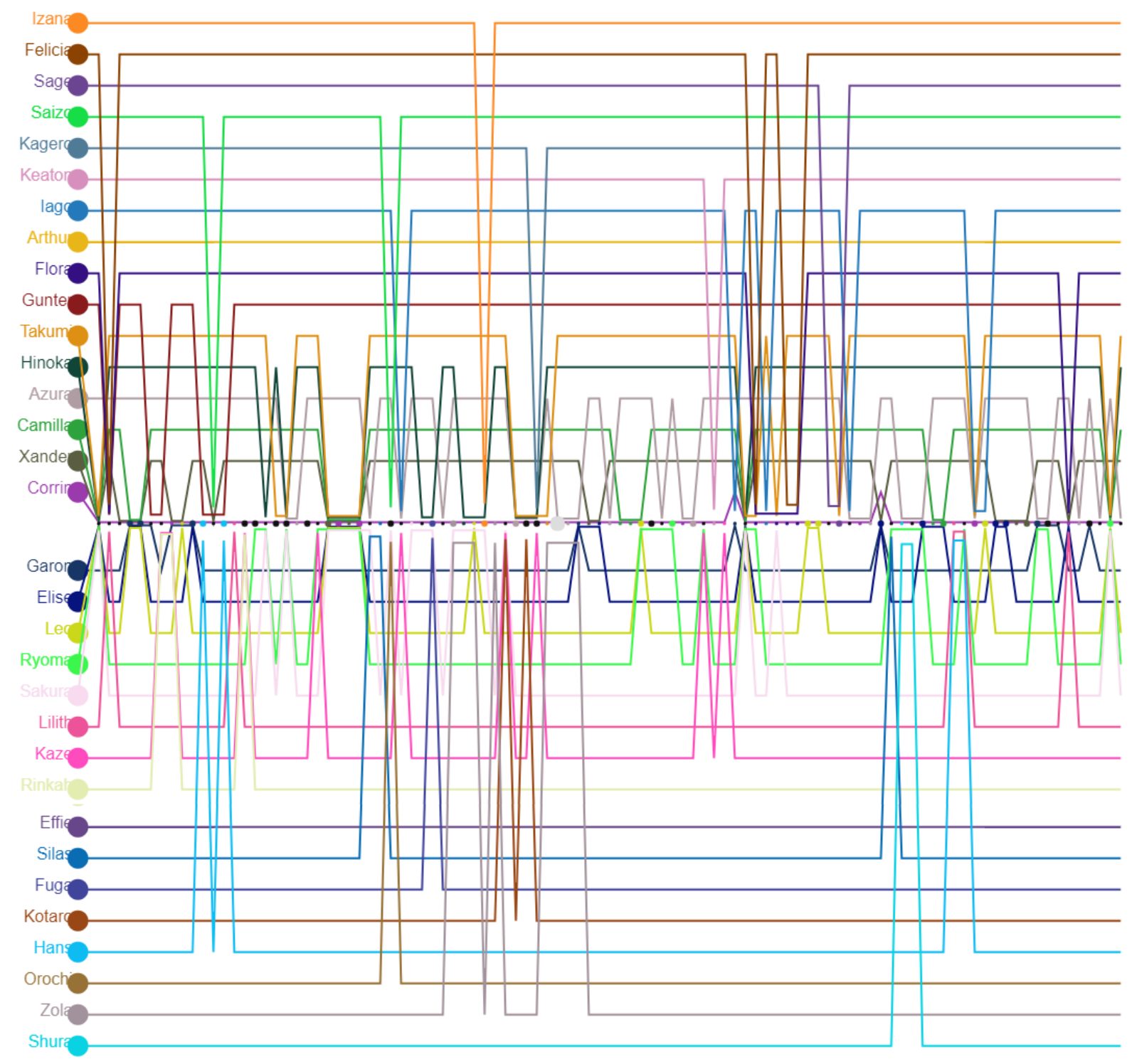


Final Fantasy X:

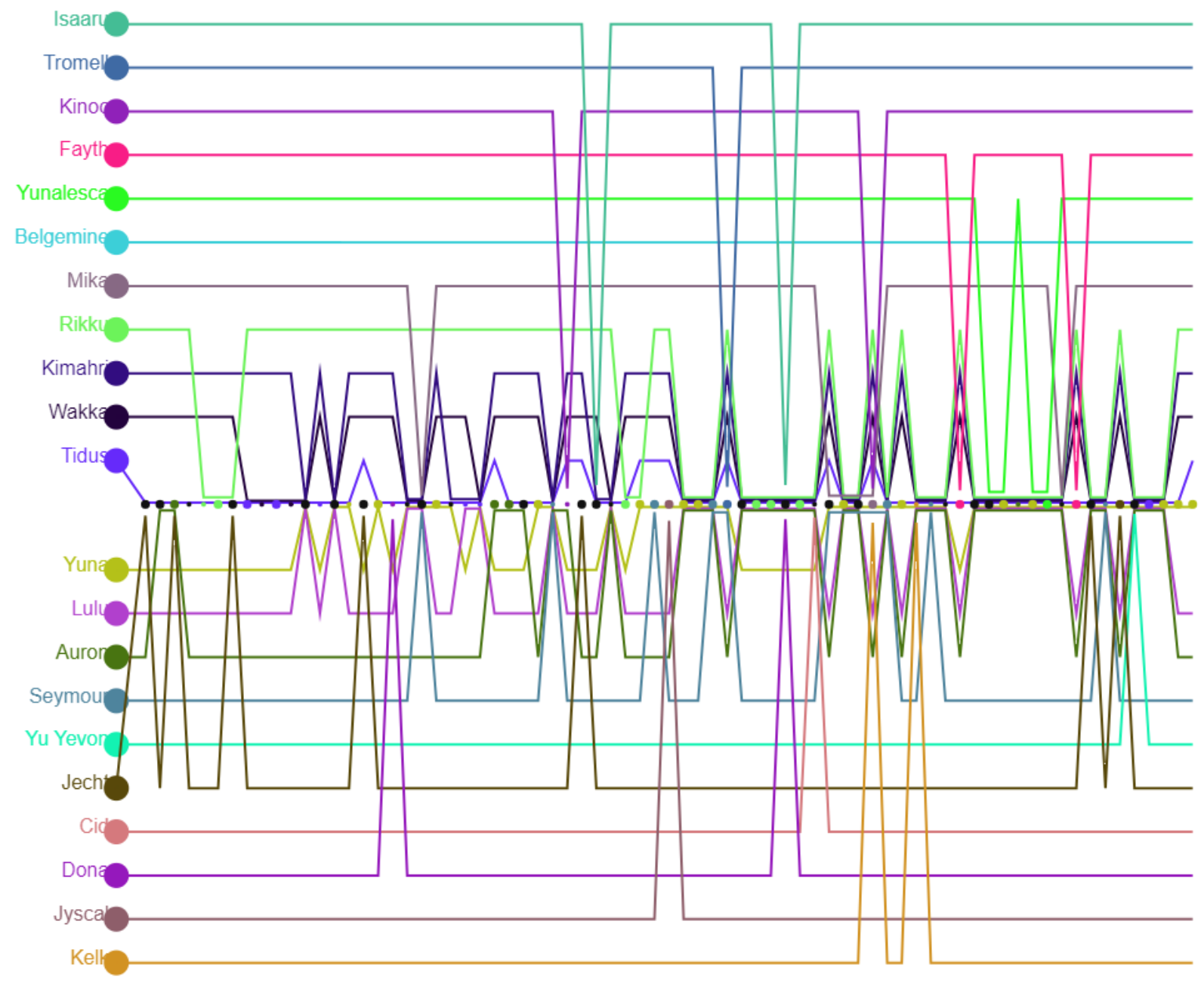




\section{Tactics Ogre: Let Us Cling Together:}

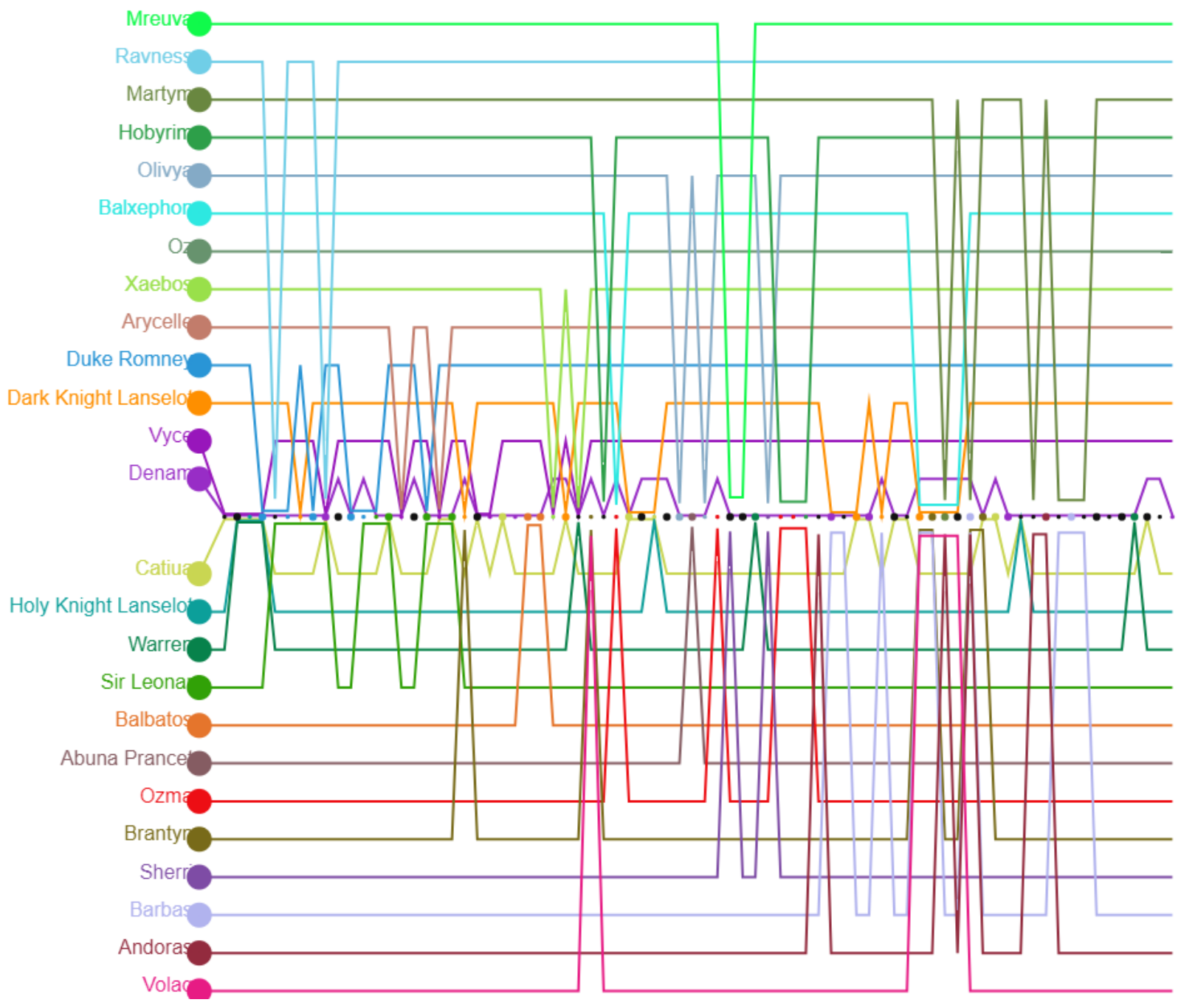


Golden Sun:

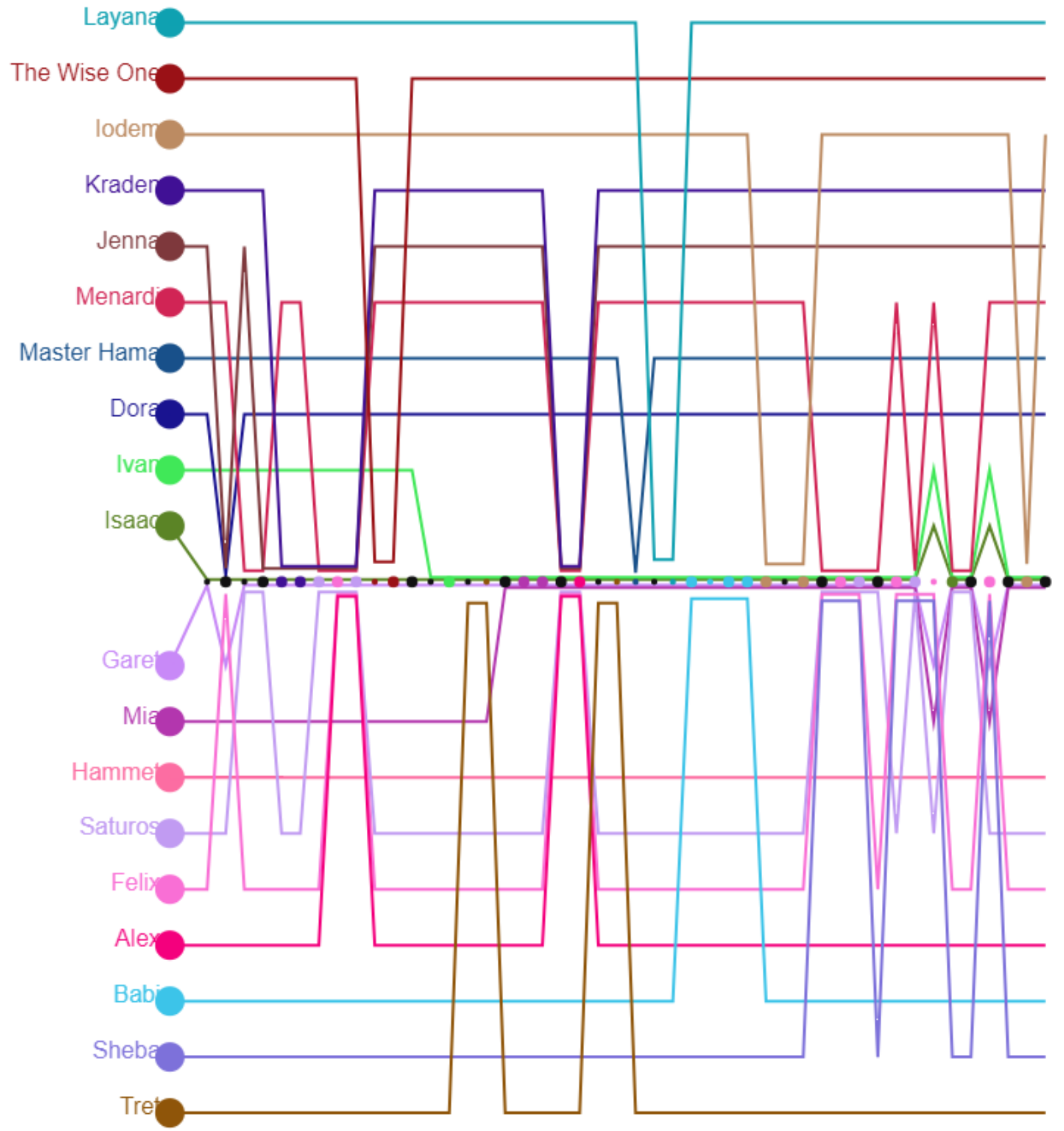


Batman: Arkham Asylum:

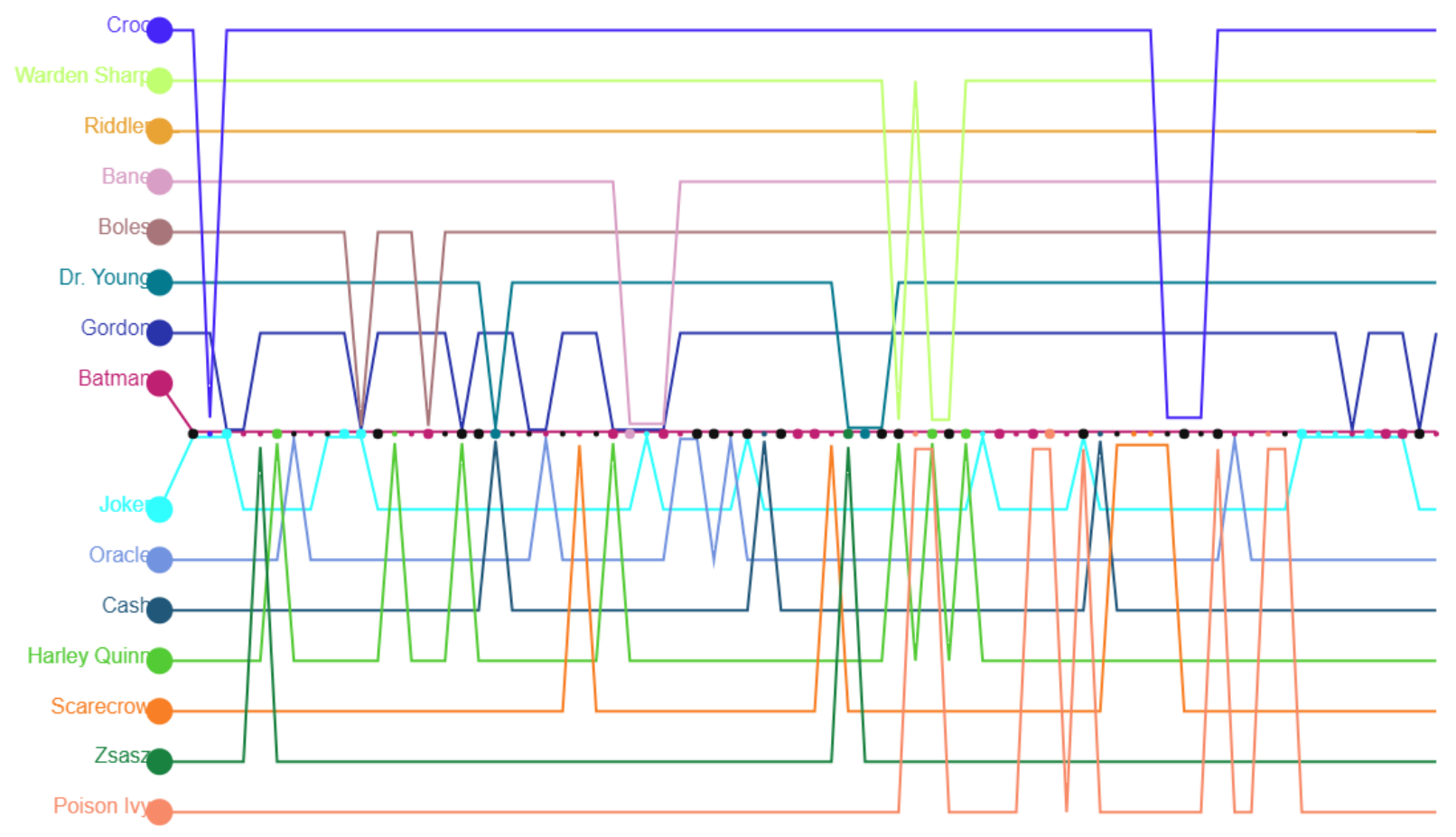


The Last of Us:

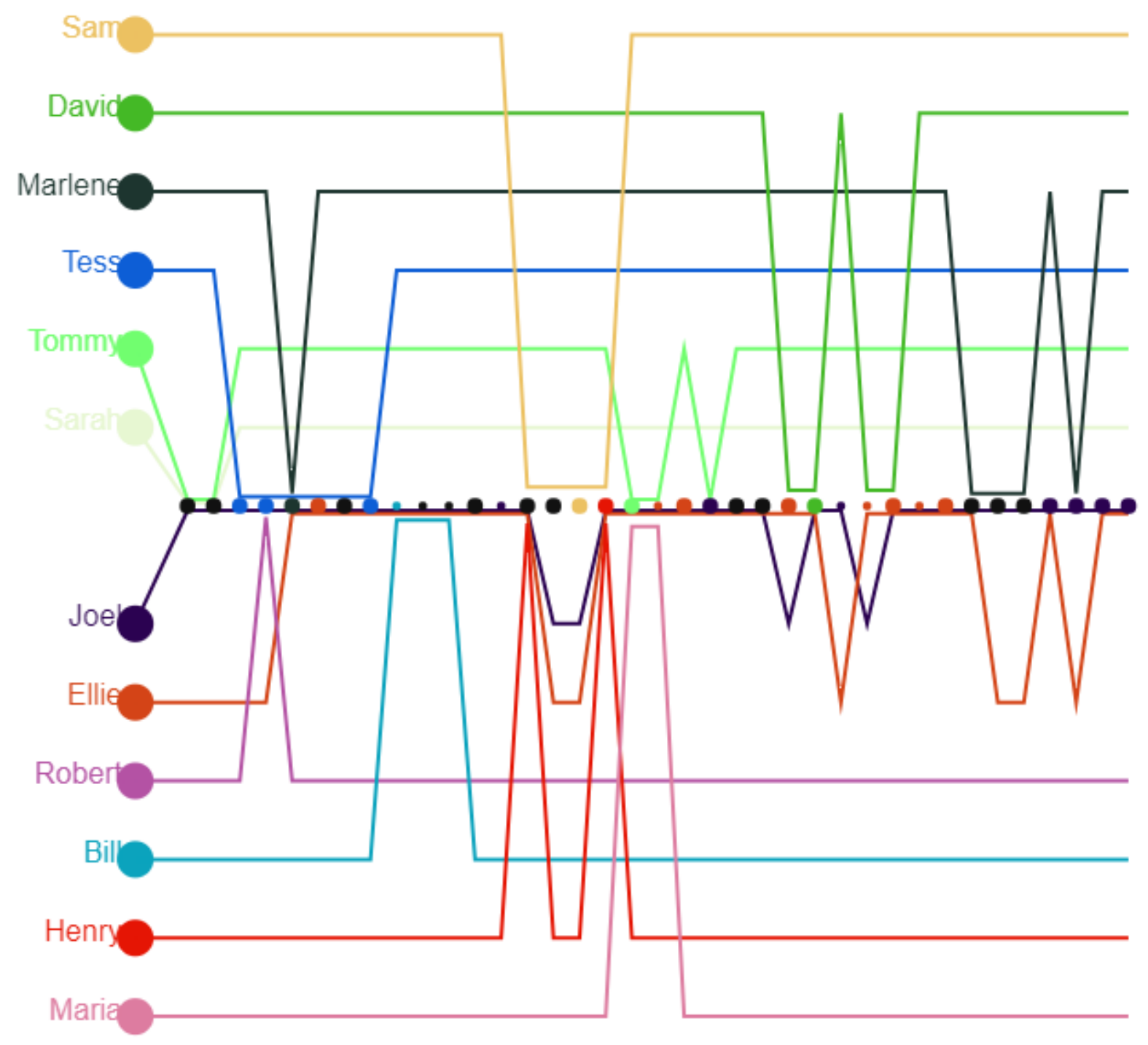




\section{Metal Gear Solid 3:}

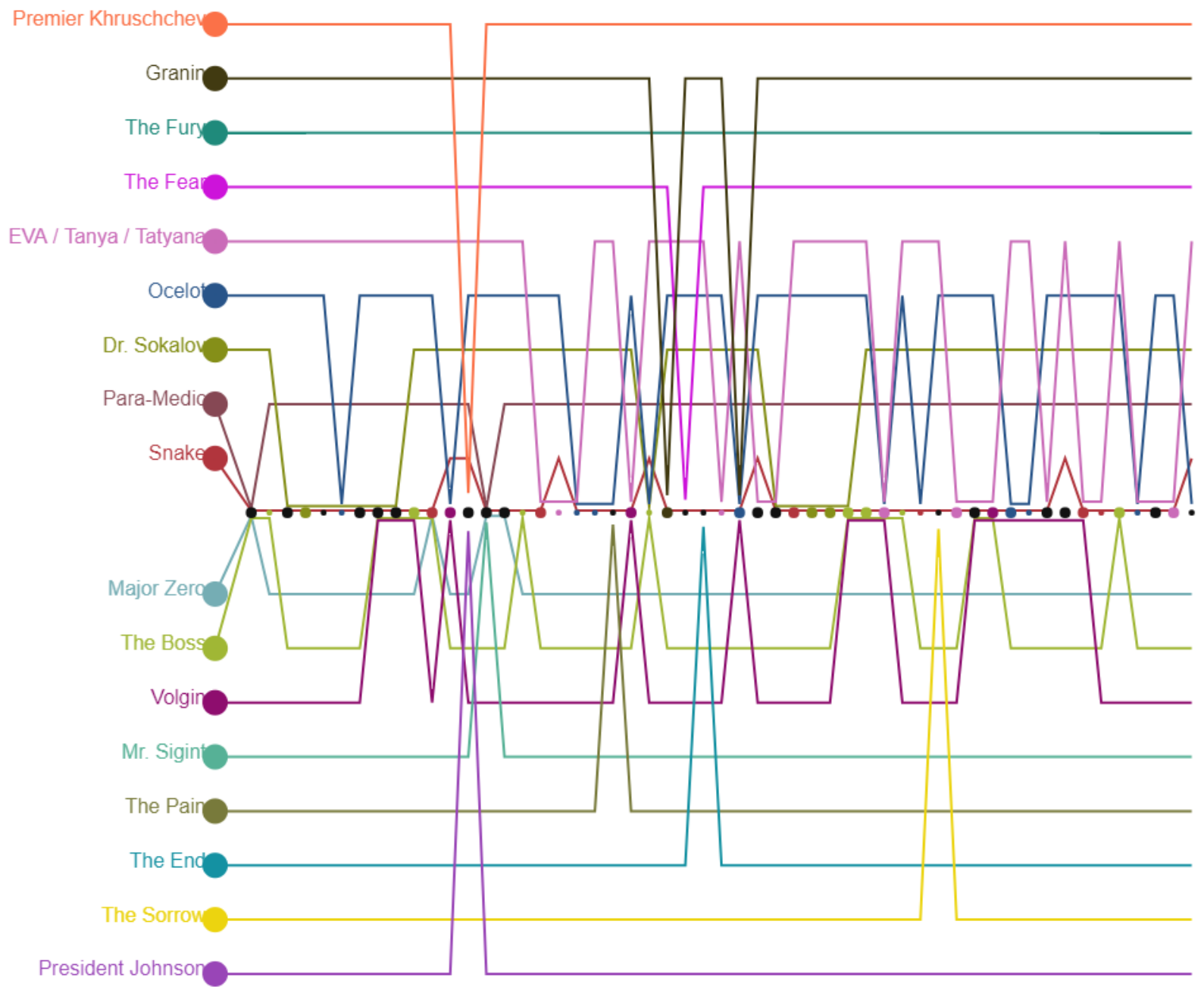


The Legend of Zelda: Ocarina of Time:

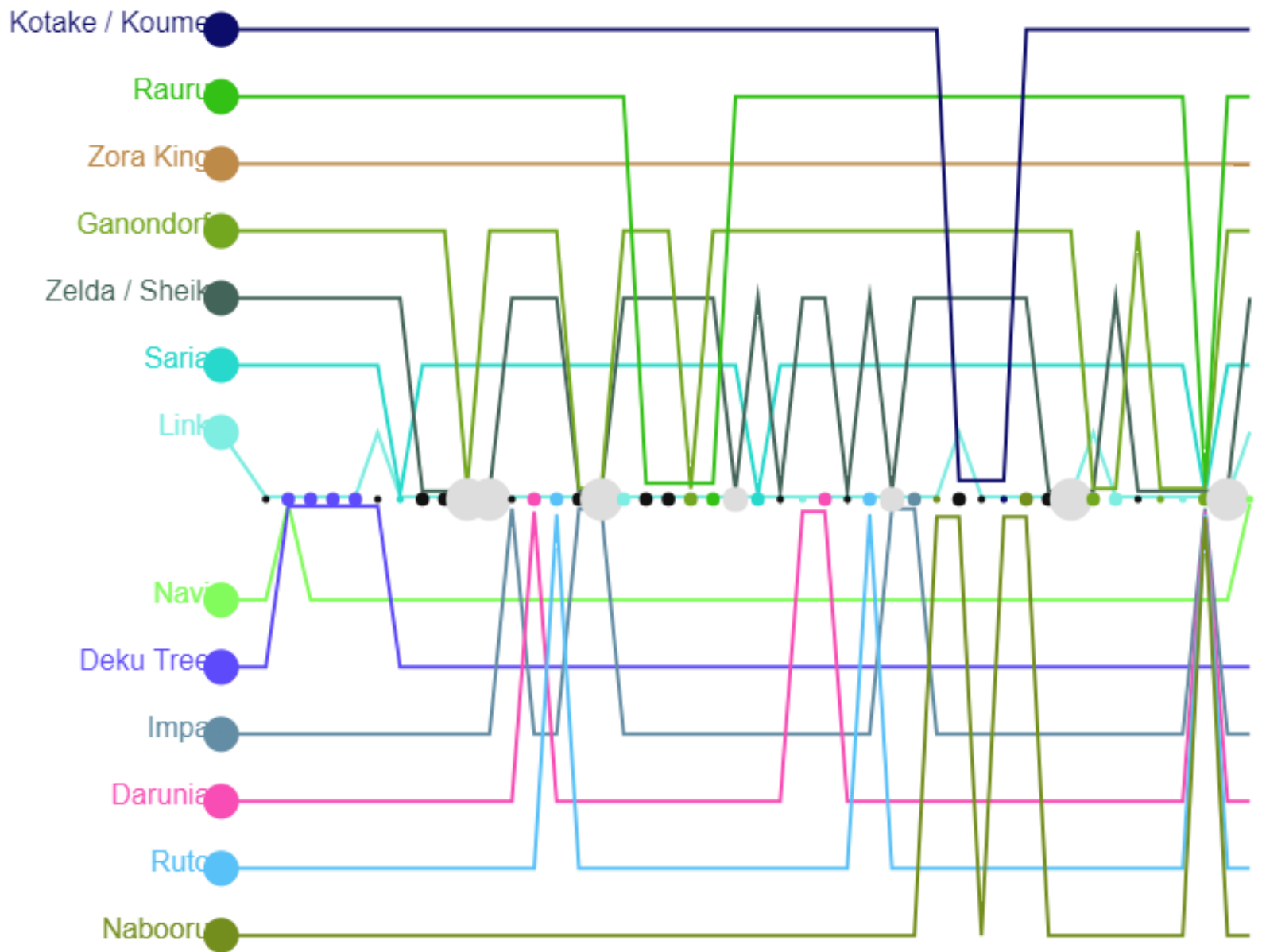




\section{Character Co-Occurrence Matrix}

Note: Data is missing for the Fire Emblem Fates campaigns.

Dark:

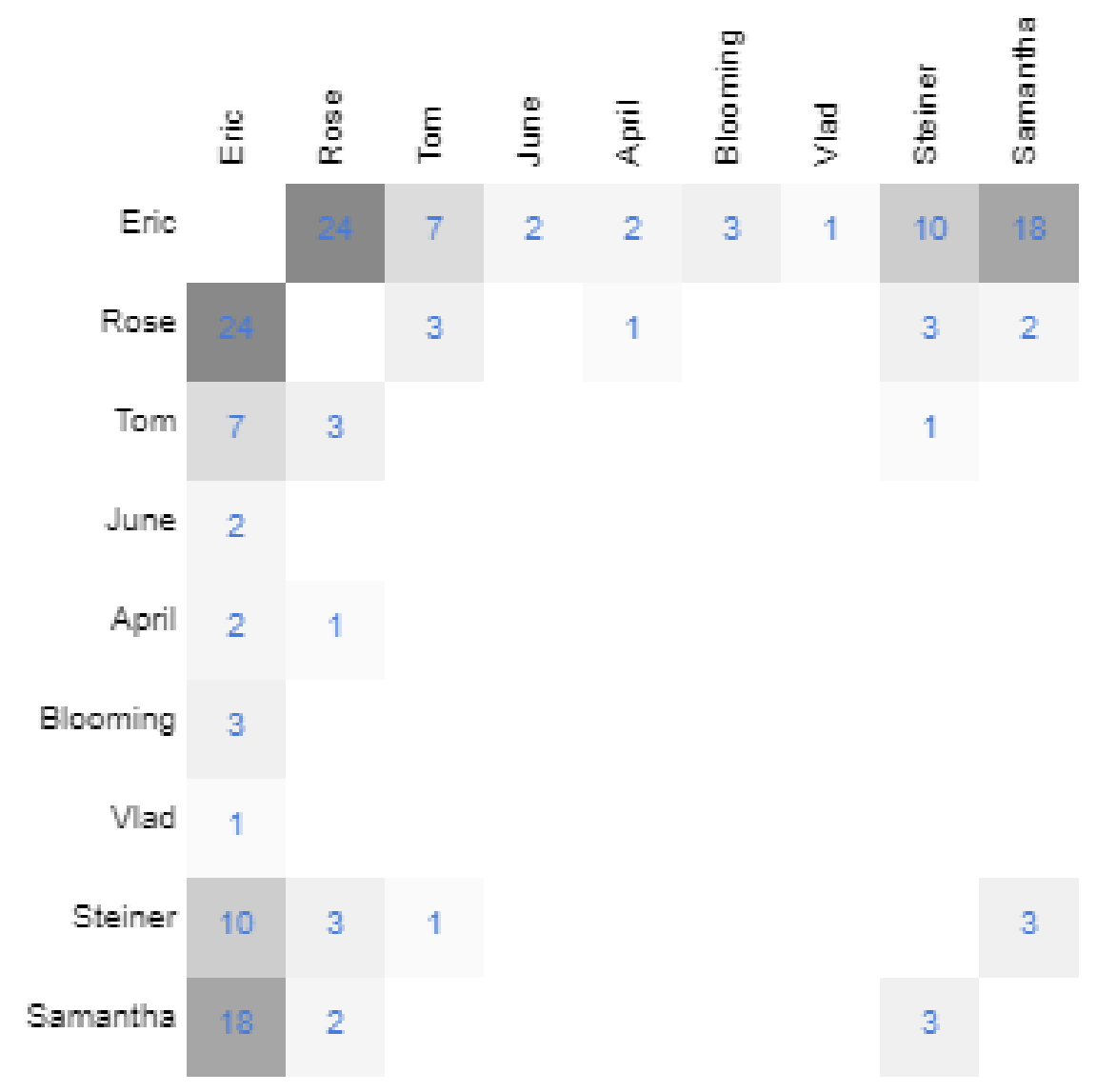

Legendary:

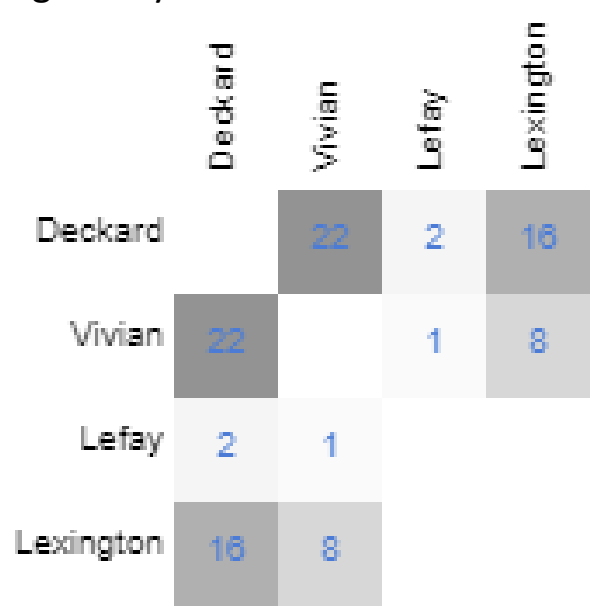




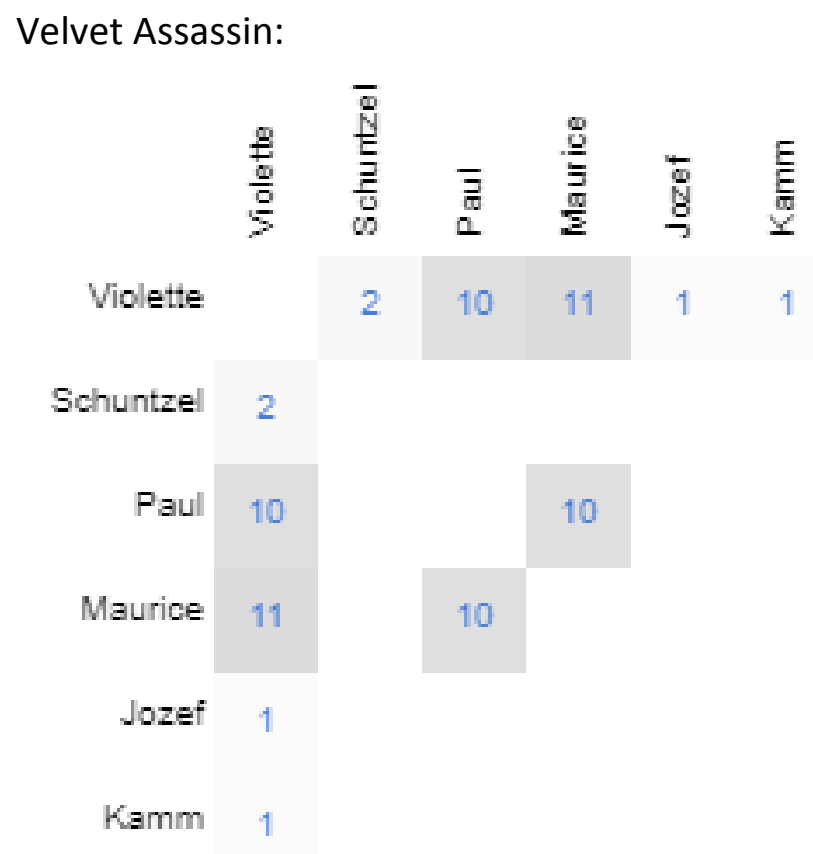

Destiny:

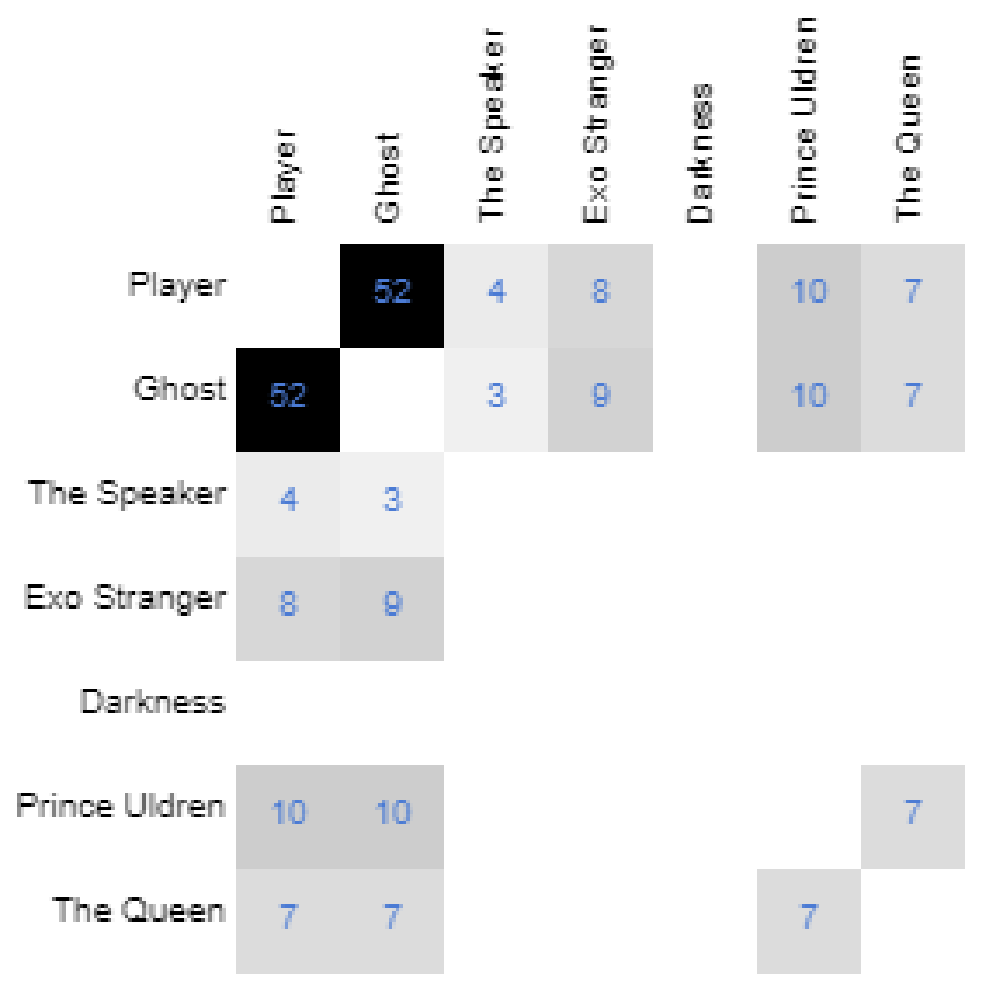


Gravity Rush:

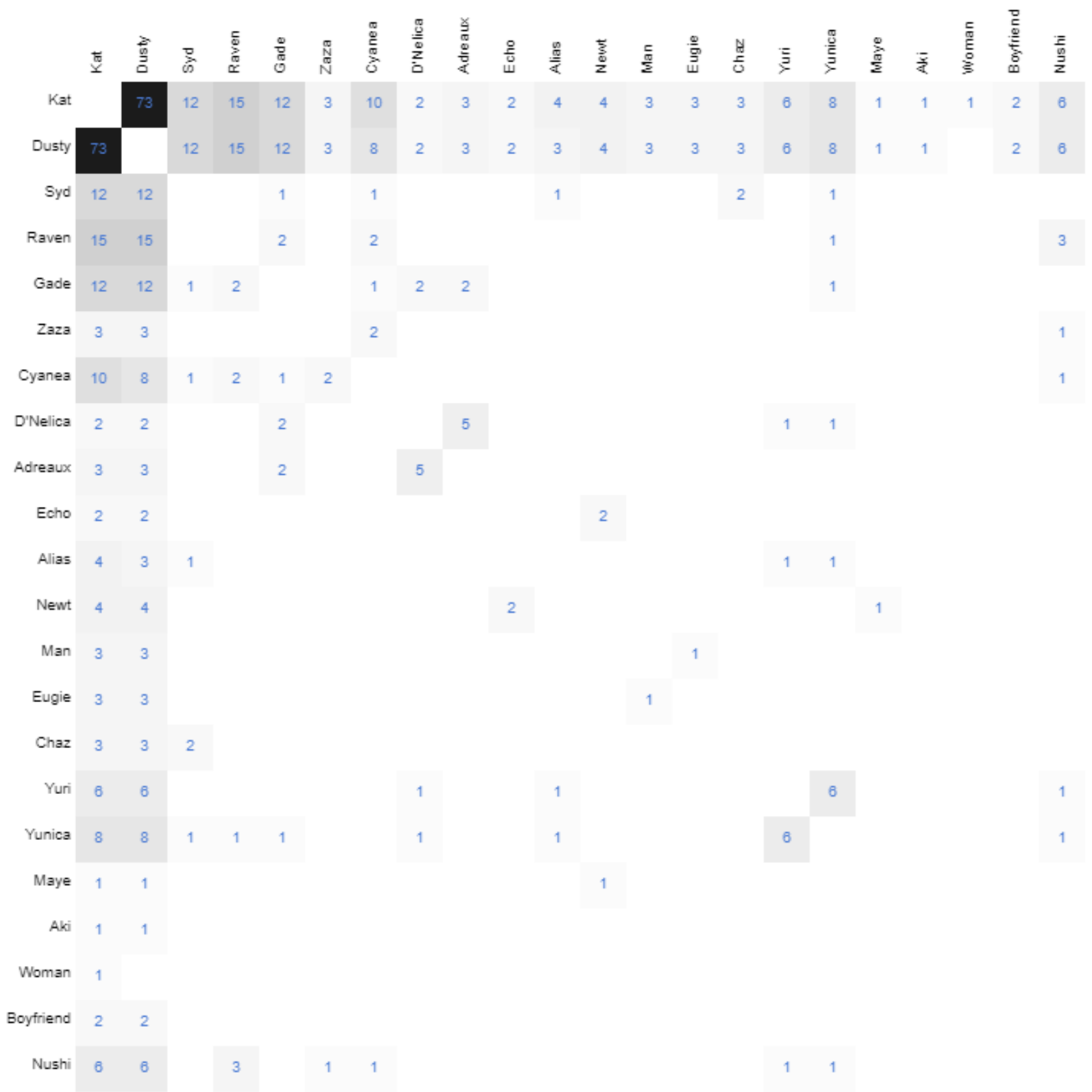


Etrian Odyssey 2 Untold:

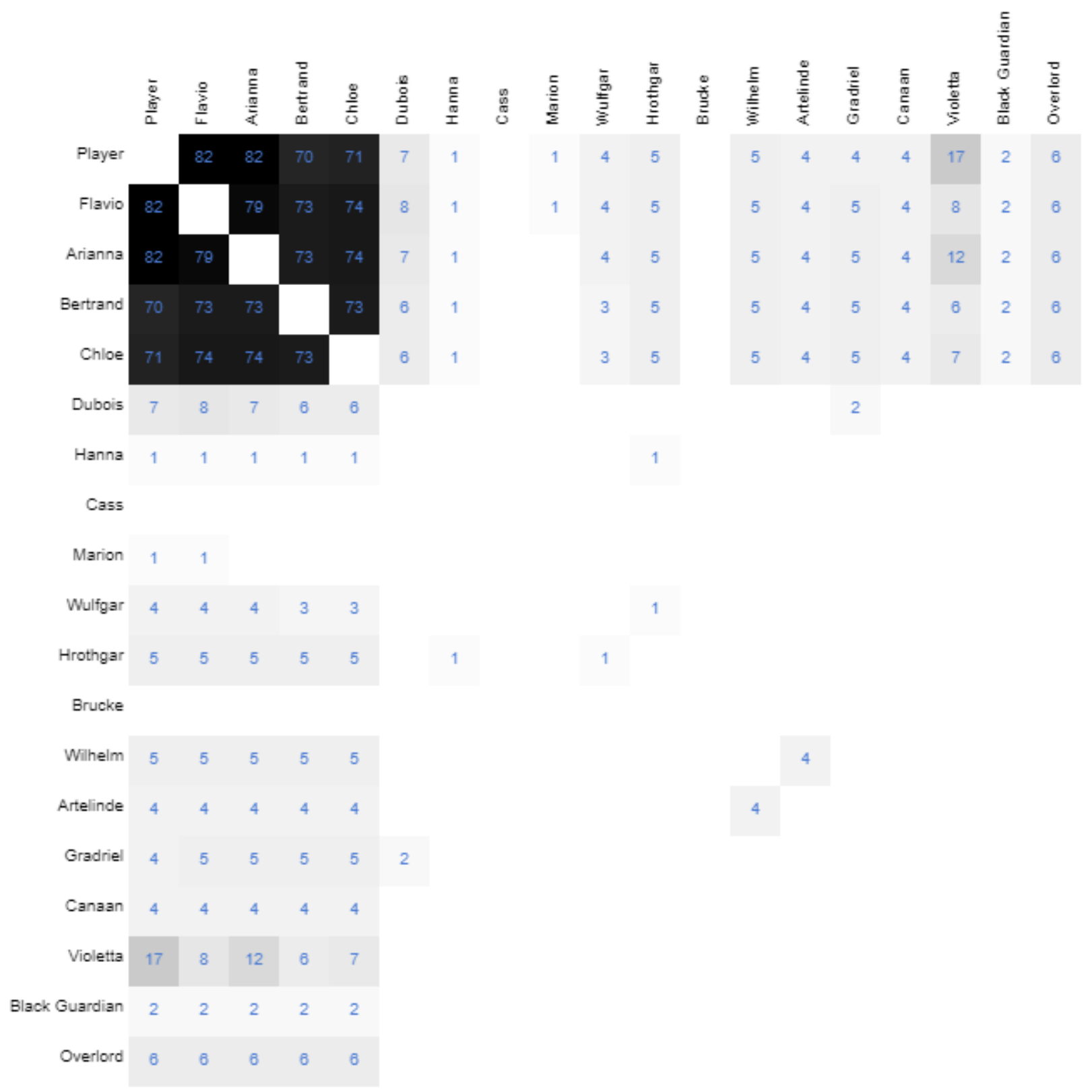


To The Moon:

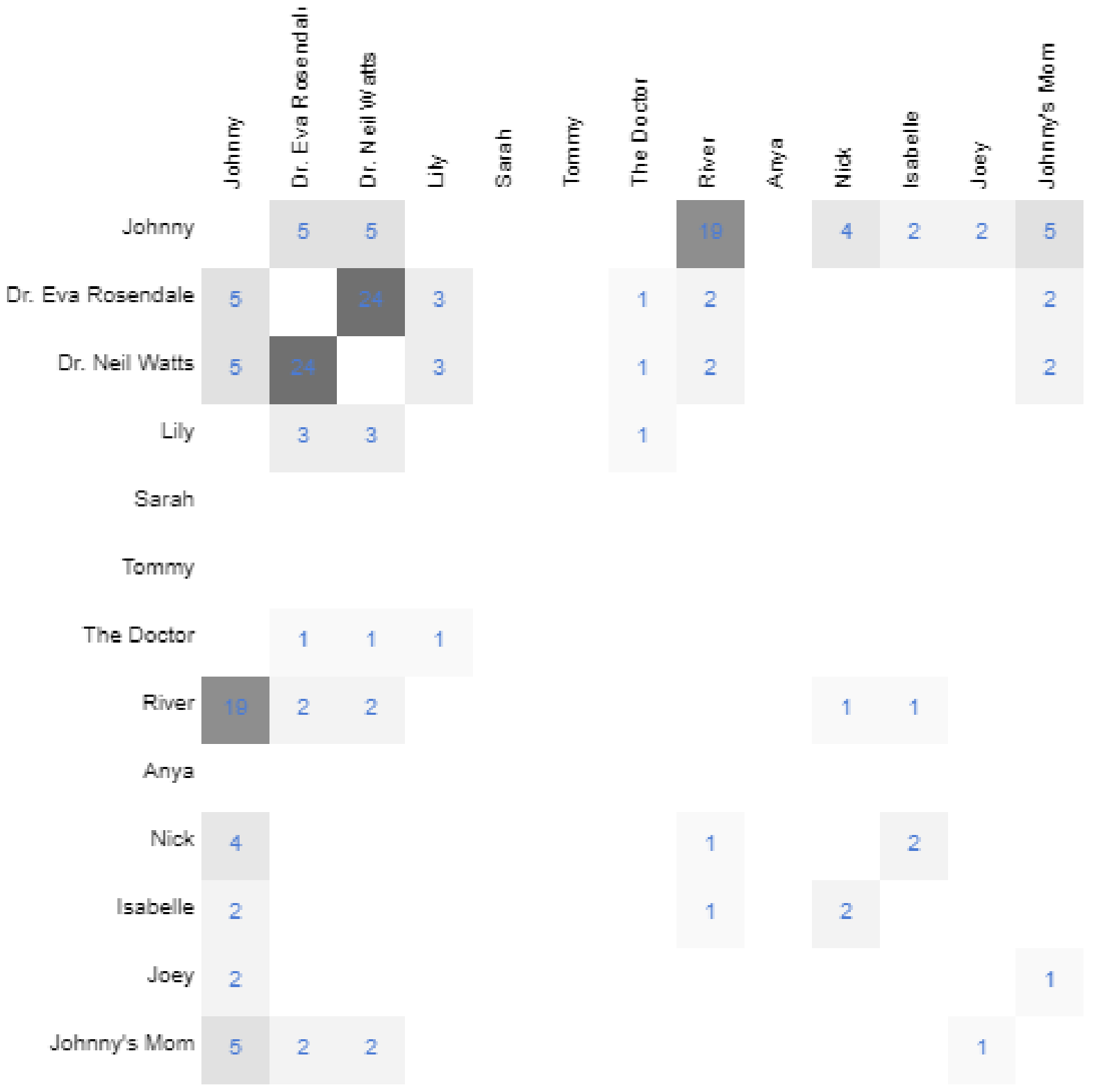


Catherine:

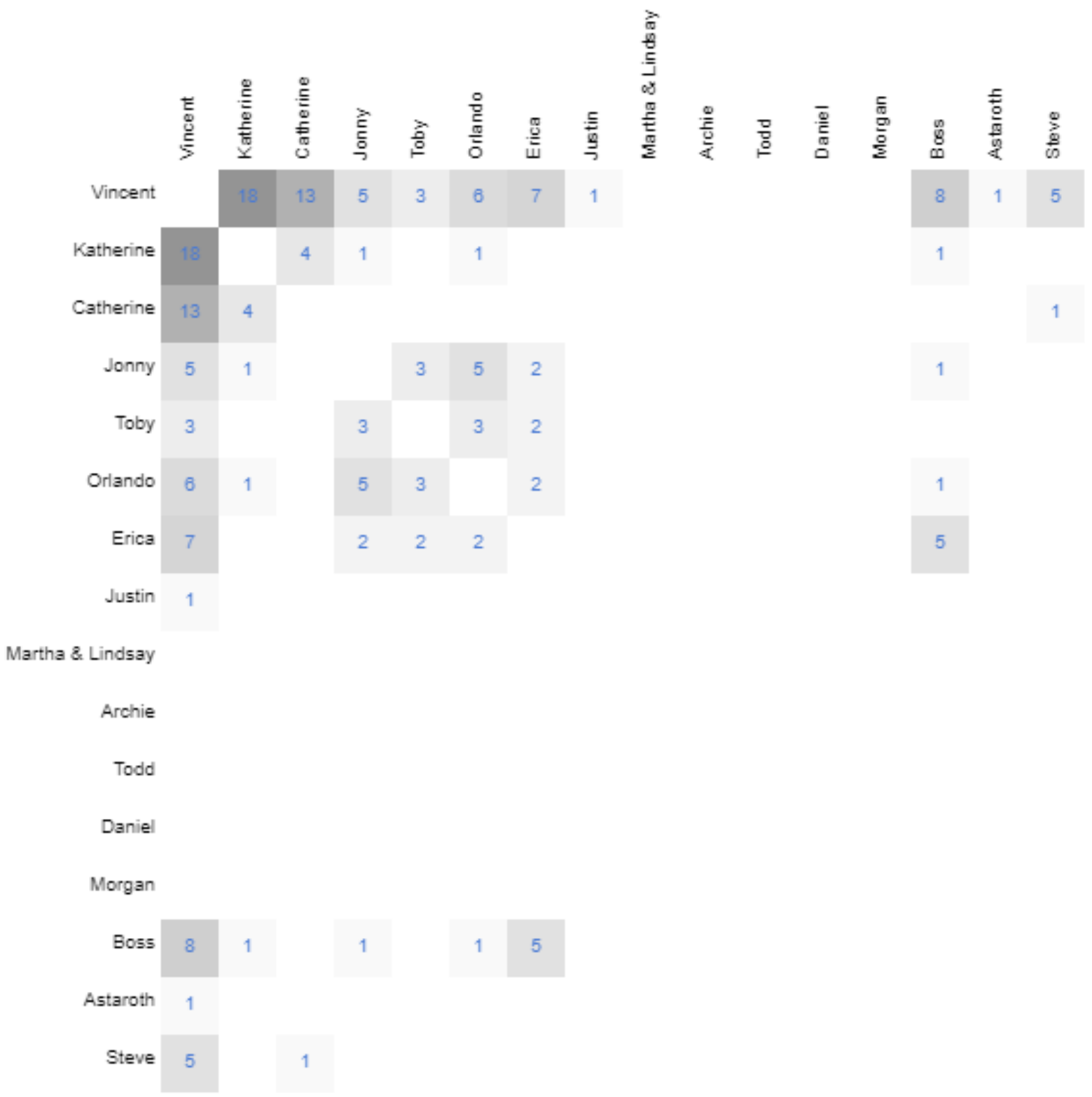


Uncharted: Drake's Fortune:

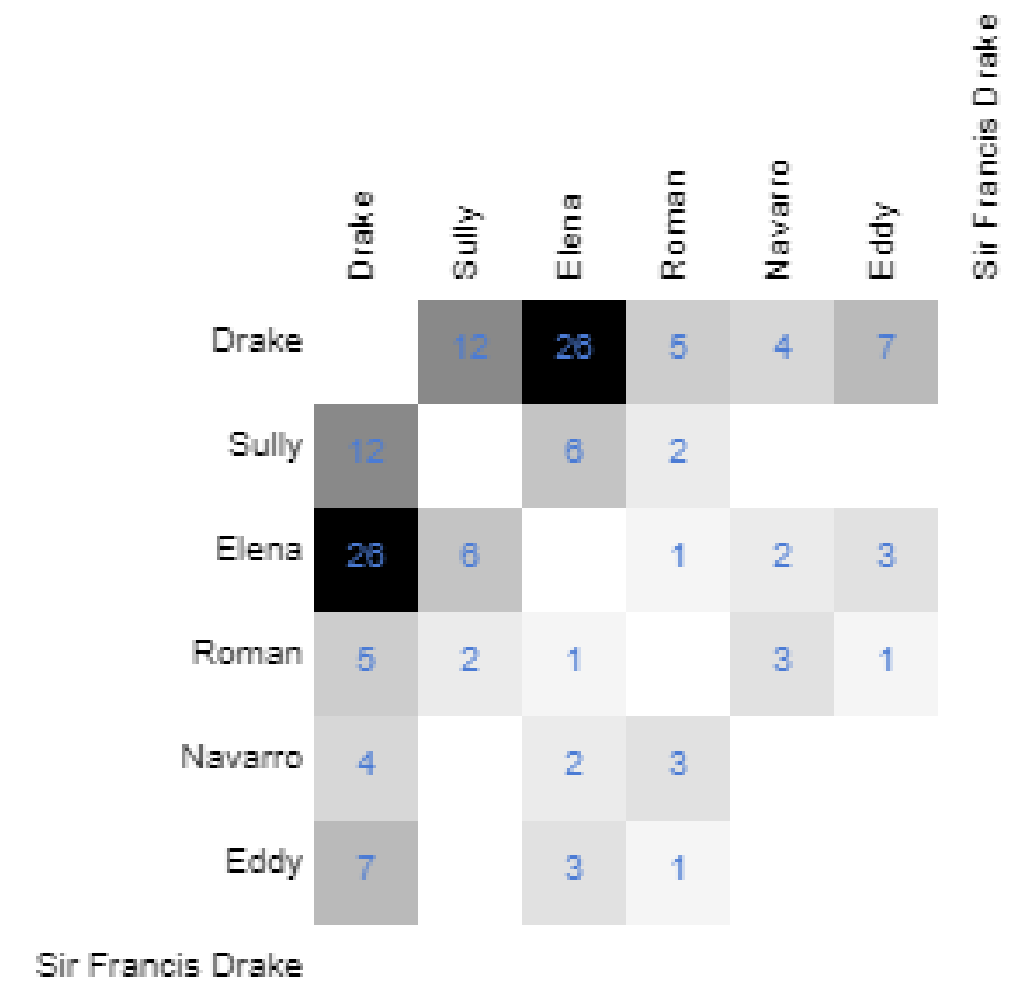


Shadow of Mordor:

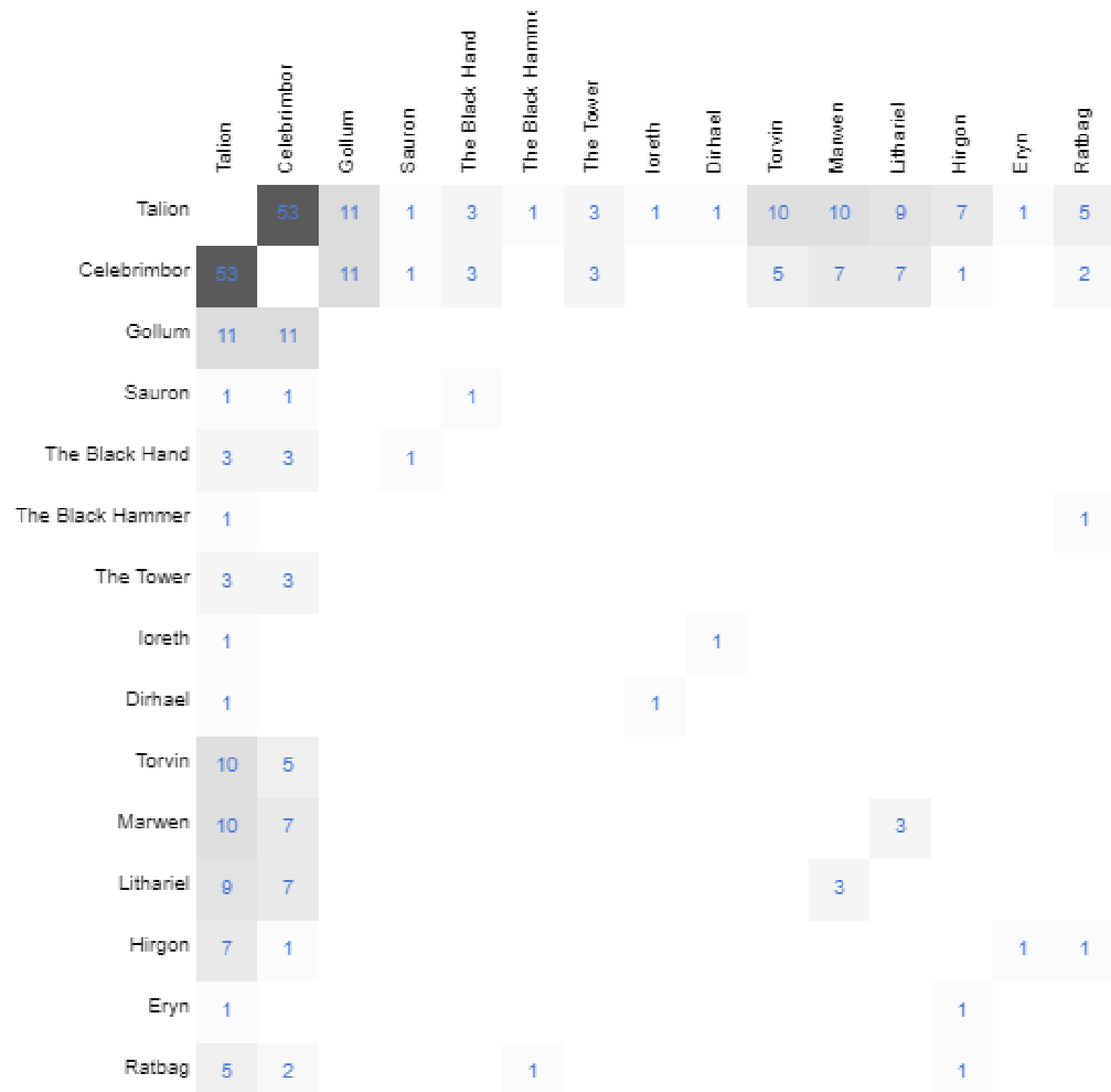


Final Fantasy X:

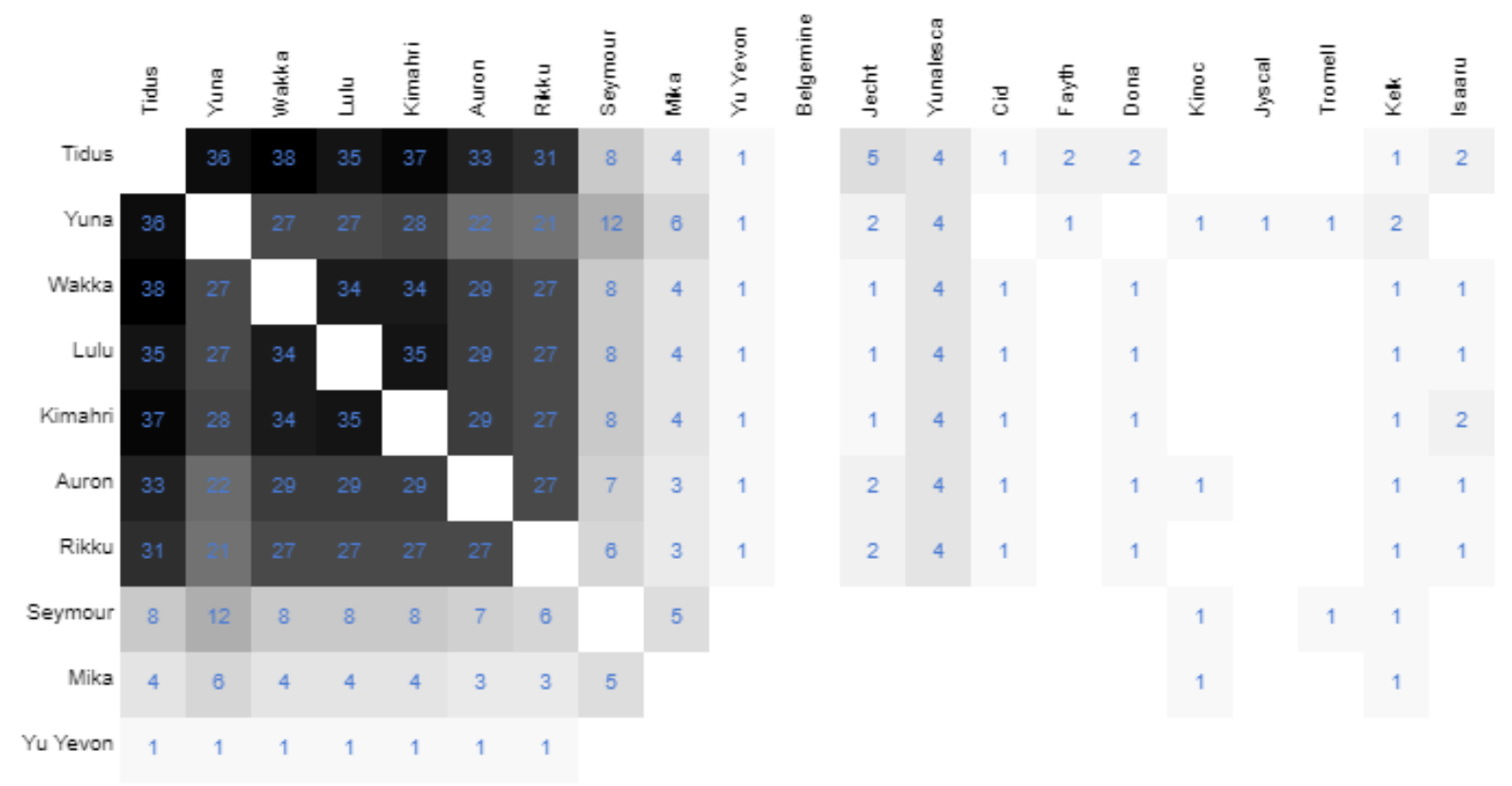

Belgemine

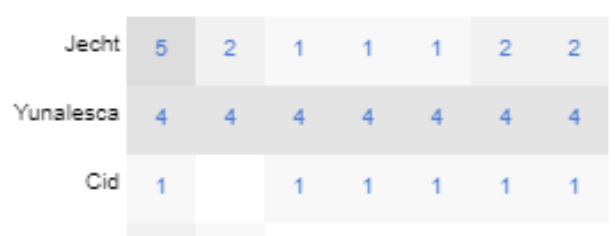

Fayth 21

$\begin{array}{lllllll}\text { Dona } & 2 & 1 & 1 & 1 & 1 & 1\end{array}$

$\begin{array}{llll}\text { Kinoc } & 1 & 1 & 1\end{array}$

Jyscal

Tromell

Kelk

Isaaru 2 
Tactics Ogre: Let Us Cling Together:

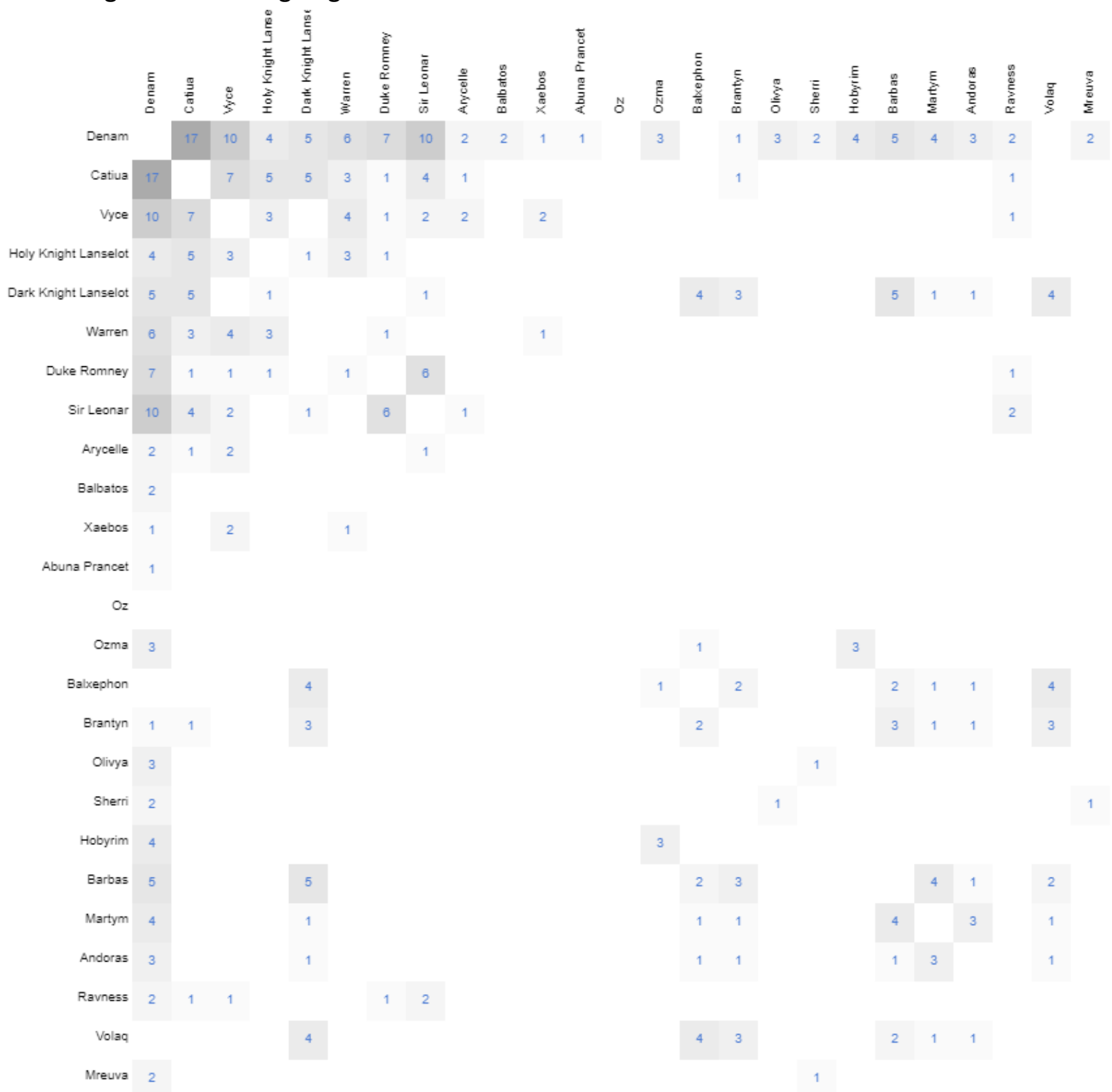


Golden Sun:

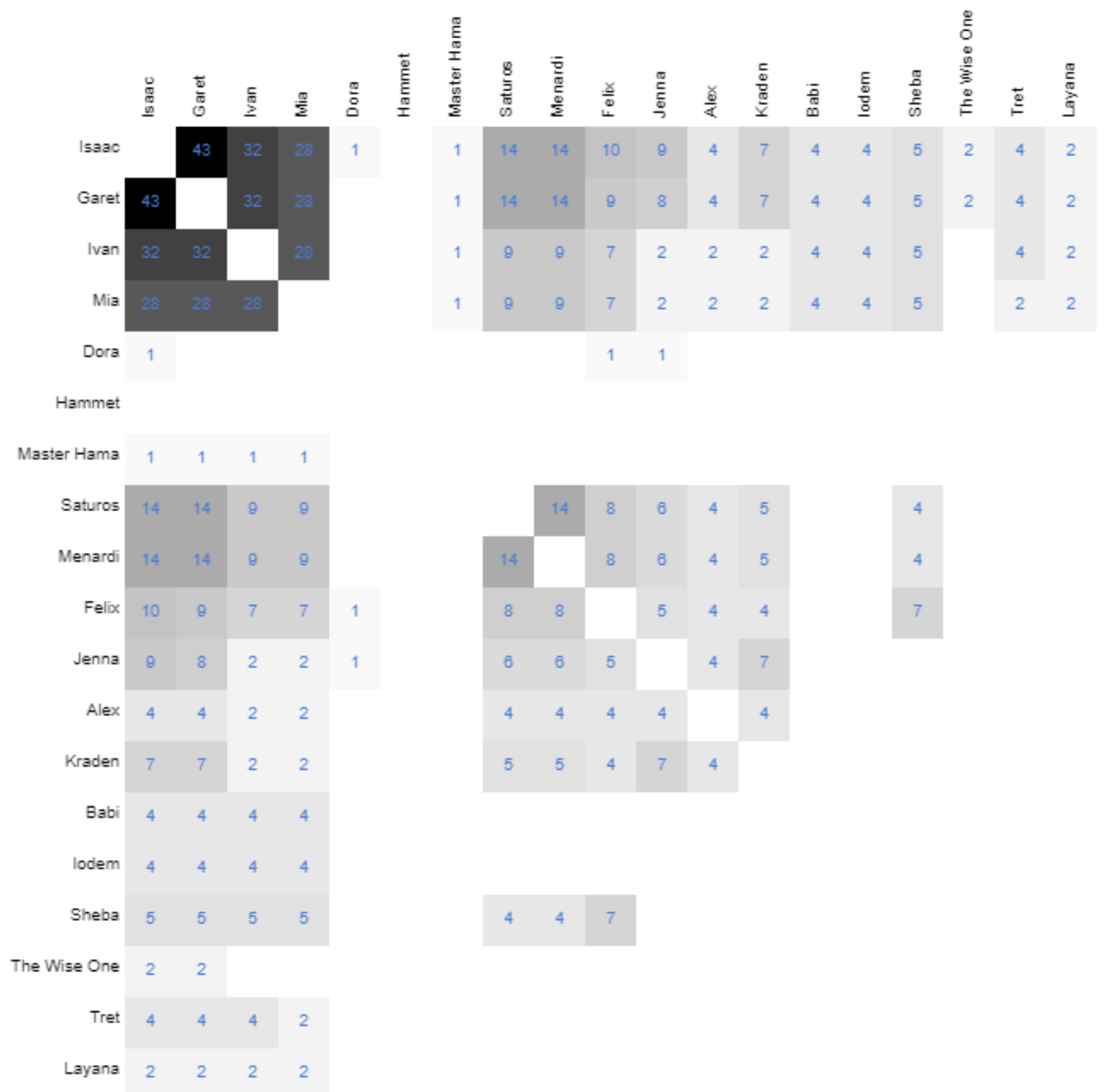


Batman: Arkham Asylum:

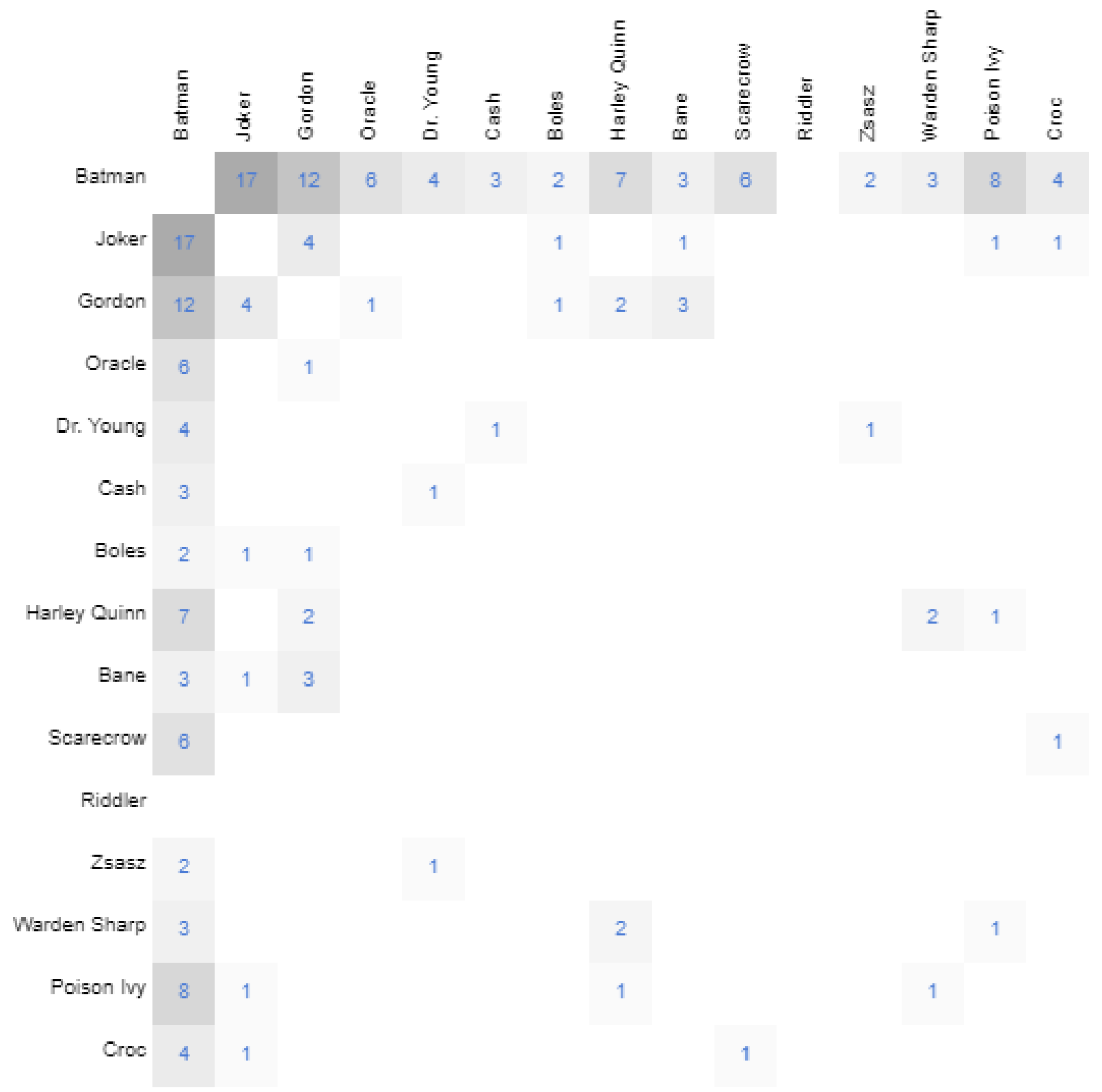


The Last of Us:

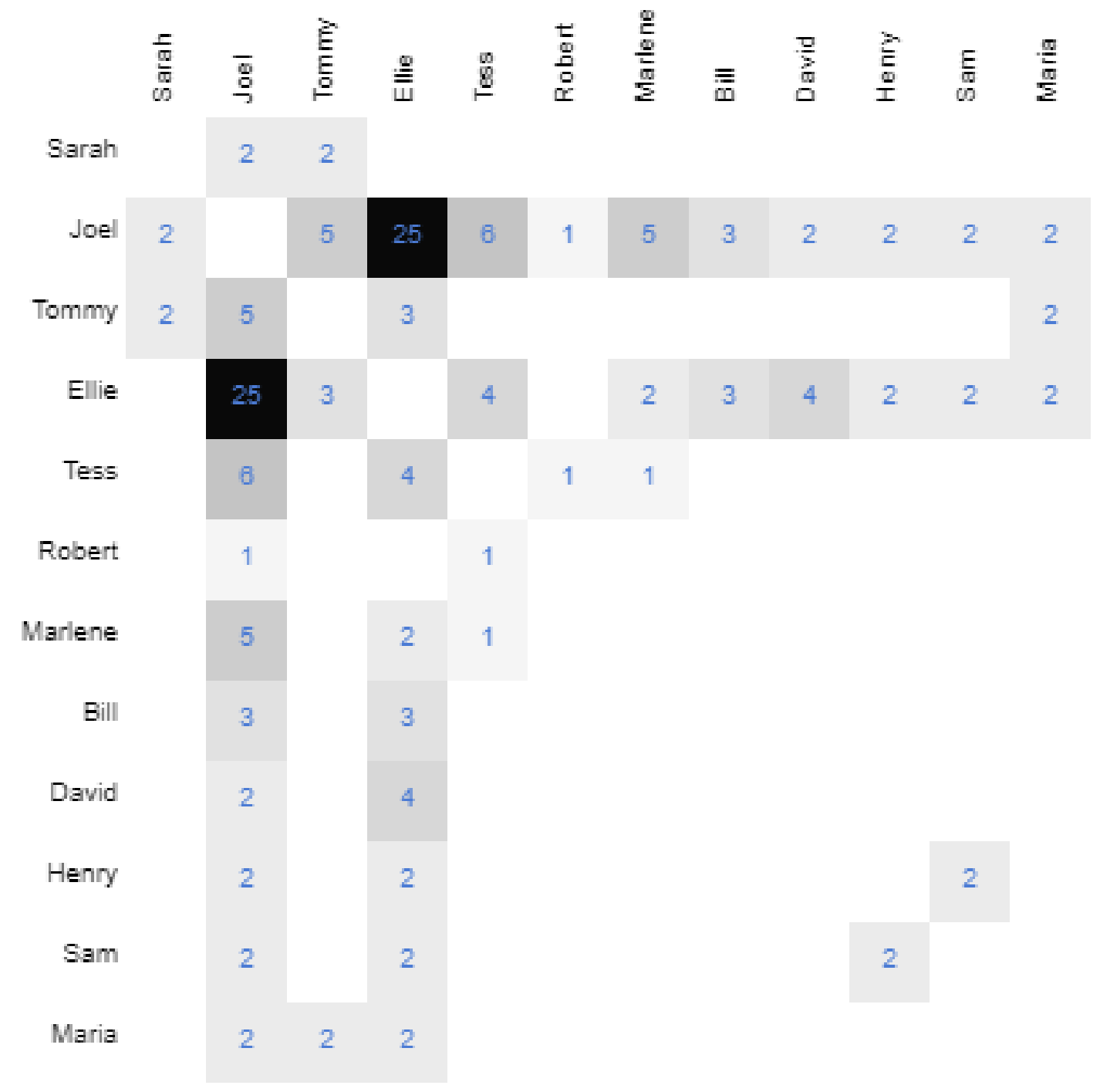


Metal Gear Solid 3:

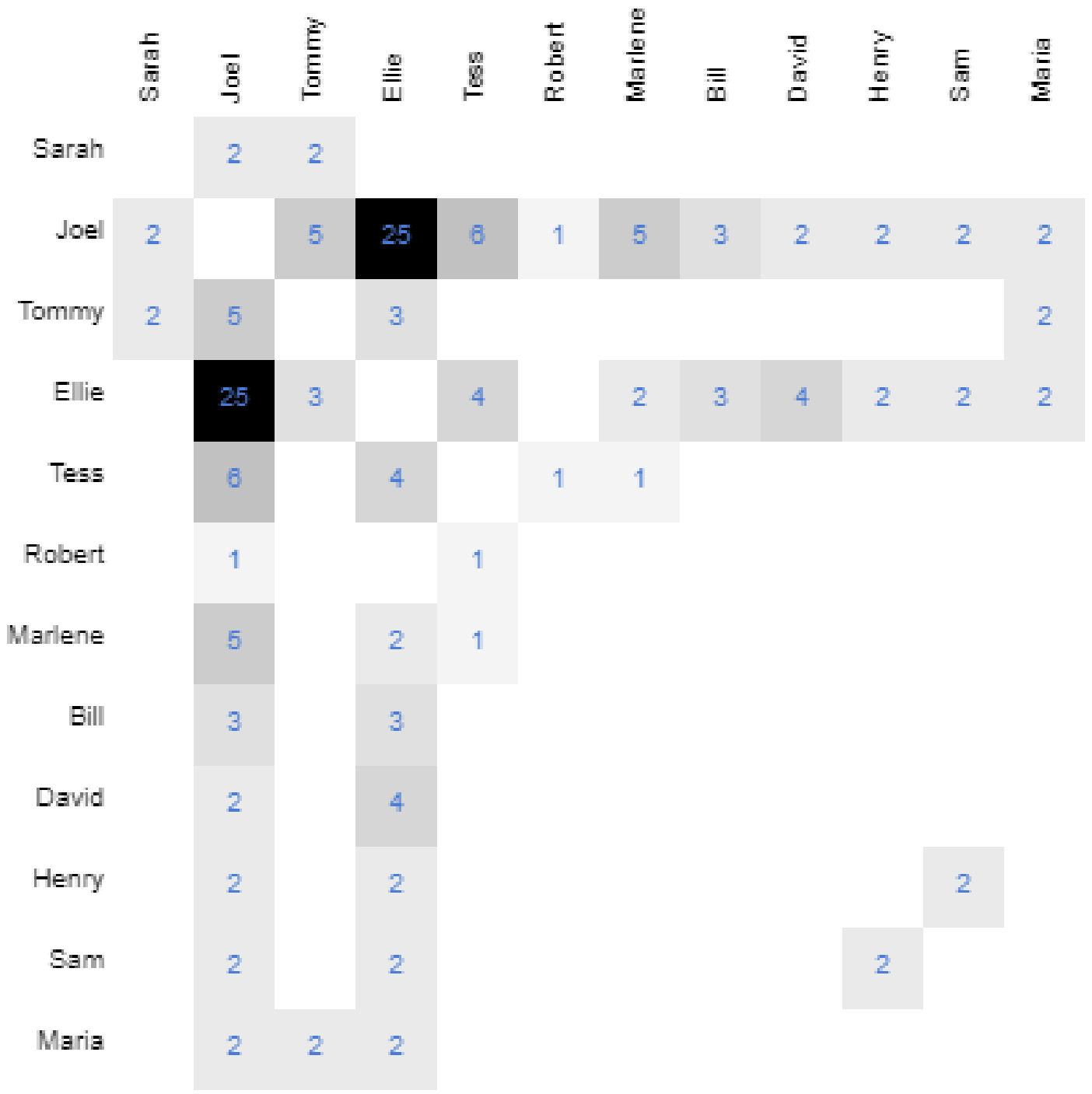


The Legend of Zelda: Ocarina of Time:

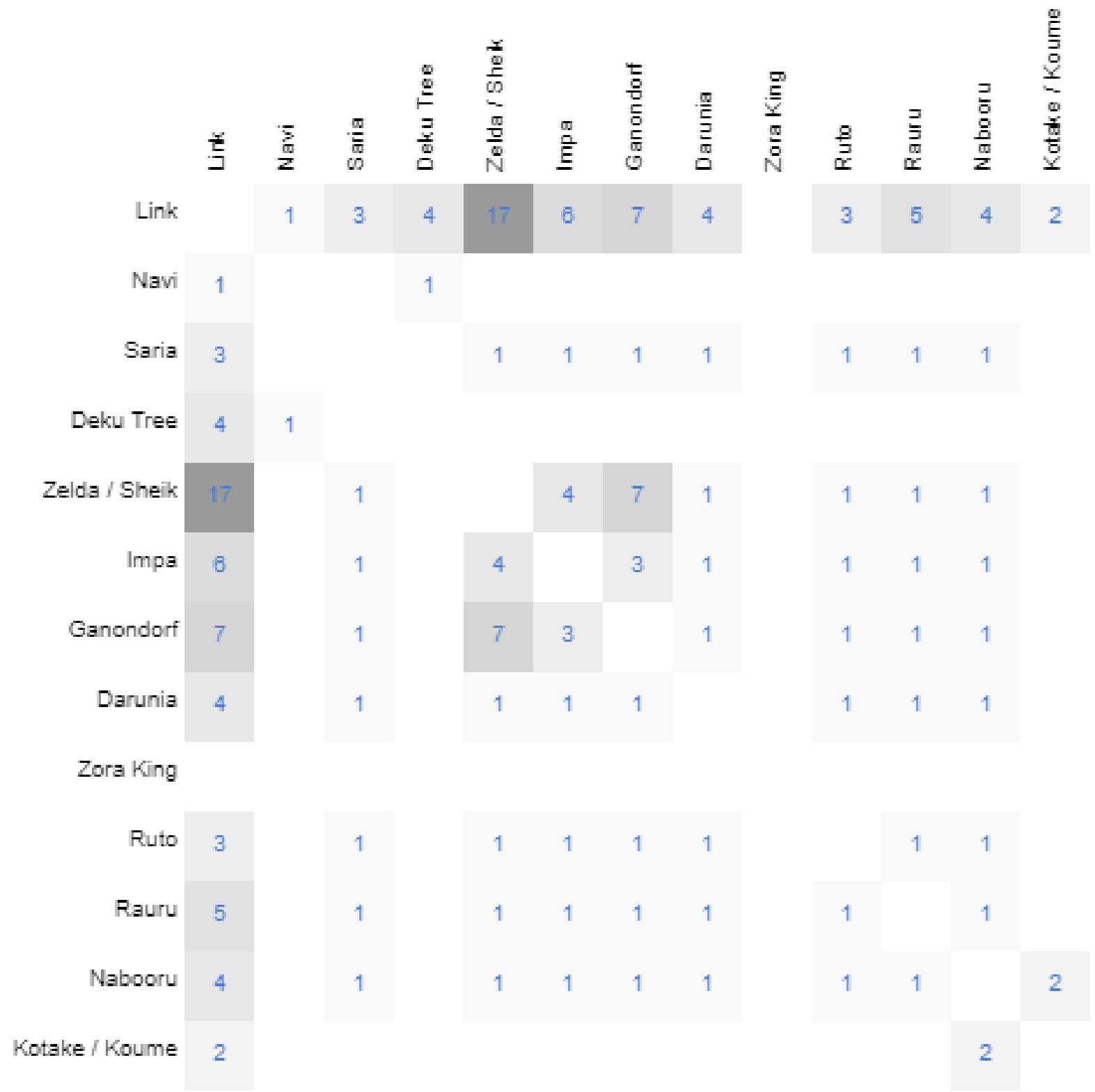




\section{Appendix 4: R Output for ANOVA Test}

$>\mathrm{df}<-$ read.table("D:/Documents/Research/CD_PD/CDPDCounts.csv", header $=\mathrm{T}$, sep $=$ ",")

$>d f$

CountPD CountCD Score gid

$1 \quad 19 \quad 3494.33 \quad 0$

$2 \quad 13 \quad 2493.751457986752$

$\begin{array}{llll}3 & 18 & 3083.67 & 206979247\end{array}$

$4 \quad 25 \quad 4889.501759301683$

$5 \quad 17 \quad 2991.671562626512$

$6 \quad 13 \quad 2996.251461322742$

$7 \quad 23 \quad 3482.001467986246$

$8 \quad 14 \quad 3781.25406526062$

$9 \quad 26 \quad 5089.60447892497$

$\begin{array}{lll}10 & 37 & 2072.601156159208\end{array}$

$11 \quad 26 \quad 4992.001617201373$

$12 \quad 40 \quad 1346.251910139021$

$13 \quad 45 \quad 5378.501437258919$

$14 \quad 52 \quad 1741.671688909195$

$\begin{array}{lll}15 & 32 & 1959.381910981236\end{array}$

$16 \quad 34 \quad 6587.90893344181$

$\begin{array}{lll}17 \quad 28 & 6883.50 & 181371315\end{array}$

$18 \quad 42 \quad 6986.251284131435$

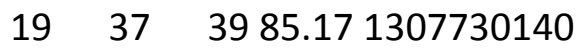

$20 \quad 41 \quad 4477.50926544296$

$>\operatorname{aov} 1=\operatorname{aov}($ CountPD $\sim$ Score, data=df $)$ 


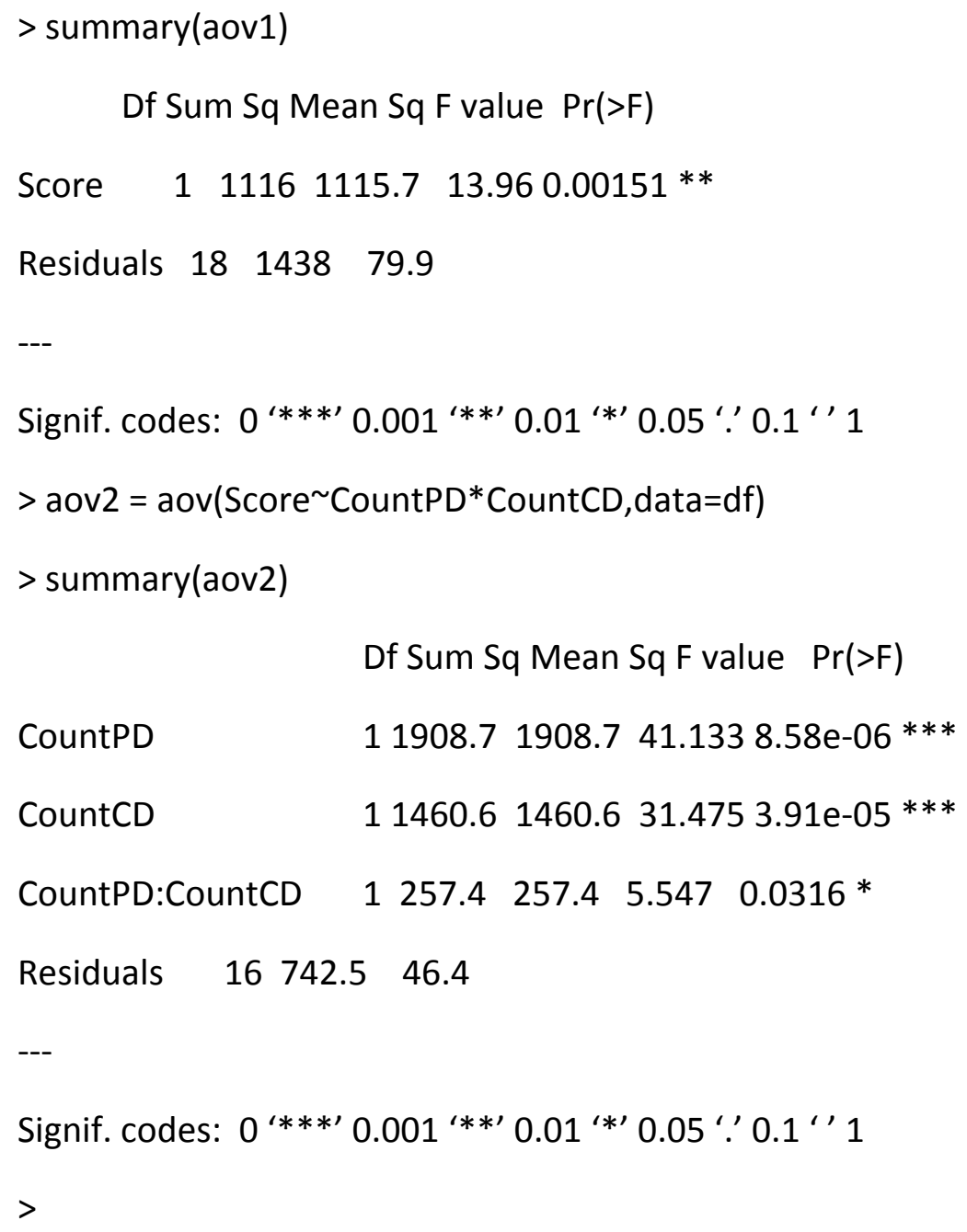




\section{Appendix 5: Character Internal / External Goal Analysis}

Internal and External goal definitions are sourced from Lisa Cron's "Wired for Story" [2]. The internal goal is abstract. The external goal is the specific action the character complets to achieve the internal goal.

\subsection{Metal Gear Solid 3}

Internal goal:

Snake: Loyalty to country and to mentor "The Boss"

The Boss: Loyalty to country

Volgin: ?

External goal: Make Volgin think she has defected in order to infiltrate and destroy Shagohod

Snake: Eliminate Boss, capture Sokalov, destroy Shagohod.

The Boss: Infiltrate volgin's ranks and destroy the Shagohod

Volgin: Overthrow KGB

Plot-Driven Event Analysis:

Plot-driven events are generally related to internal goals.

\subsection{The Last of Us}

Internal goal:

Ellie: Do what she can to help an antidote be found 
Joel: Regain the connection he had with his daughter? Do right by Tess? Loyalty to those he loves?

External goal:

Bring Ellie to the Fireflies.

When push comes to shove, Joel's internal goal conflicts with this so he rescues Ellie from death, at the cost of the antidote.

Plot-Driven Event Analysis:

Plot-driven events are usually to impress the severity of the state of the world - people being infected or injured or killed. All of this shows the pressure of infection, and makes Ellie's goal of finding a cure seem important to the player. It also shows the human connection between the survivors, and especially joel / ellie, which furthers our understanding of Joel's internal goal to remain loyal to those he cares about (such as Tess, Ellie).

\subsection{Uncharted: Drake's Fortune}

Internal goal: Discover the truth behind his ancestor's actions?

External Goal: Find the treasure before Roman

Plot-Driven Analysis:

Plot-Driven events are generally concerned with Francis Drake and what happened to him in the past, which is related to Drake's desire to learn about his ancestor.

\subsection{Final Fantasy X}


Internal Goals:

Yuna: Duty, peace for Spira

Tidus: Redemption with his Father, ???, get back home

External goals:

Yuna: Perform the Final Summoning. Later on, defeat Sin for good

Tidus: Help Yuna, fulfill the dream of the fayth

Plot-Driven Analysis:

PD Events seem to be mainly world building (Tidus learning about sin / fayth / aspects of the world). Similar to The Last of Us, they illustrate the state that the world is in, and provide motivation for Yuna to continue along with her goal of achieving peace for Spira

\subsection{Golden Sun}

External goal: The Wise One tasks Isaac and Garet with retrieving the Elemental Stars from Saturos and Menardi, in order to save the world.

This aligns with their internal goal of rescuing their friend Jenna from Saturos and Menardi.

Ivan's goal of either returning to Kalay or assisting his master are both unattainable, so he joins up with Isaac and Garet in order to prevent more bad things from befalling others.

Mia's goal to protect the Mercury lighthouse turns personal when Alex, part of Saturos and Menardi's team, is revealed to have been her former teammate. After the Mercury lighthouse 
is lit she joins up with Isaac and Garet in the hopes of stopping him from lighting any other lighthouses.

Many of the antagonists goals are not revealed until Golden Sun: The Lost Age. The two games make up a single story - Golden sun ends well before the midpoint of the overall narrative.

The main villain's internal goals are to stop the destruction of their village from the slow erosion of the world. Felix's internal goal is initially to show his gratitude to those who saved him, later to protect his sister.

Plot-Driven Analysis:

Plot-Driven events generally show the negative results of Saturos / Menardi's work, such as with Tret's illness, or for Ivan, provide information about his quest to save Master Hammet.

\subsection{The Legend of Zelda: Ocarina of Time}

Link's initial goal is to save the dying Deku Tree. The Deku Tree then sends him to Princess

Zelda, who tasks him with retrieving the 3 spiritual stones in order to stop the evil Ganondorf from retrieving the Triforce.

Link is inadvertently rocketed forward in time 7 years, where he discovers Hyrule in ruins under Ganondorf's control. His goal then becomes to defeat Ganondorf in order to save the realm.

Internal goal: Fit in, find his place in the world

External goal: Stop Ganondorf

Plot-Driven Analysis: 
PD Events provide the "next step" in Link's quest - example: the objective to find the spiritual stones, or the revelation of his transformation into adult link, or his ability to travel back and forth through time. The PD events therefore seem to be more related to the external goals than the internal goals, although link's internal goals are slightly fuzzy.

\subsection{Catherine}

Vince's initial internal goals simply revolve around getting through his day - dealing with his relationship problems and balancing his tiredness from his ever increasing dreams. After he meets Catherine and cheats with her, his goal becomes to somehow deal with the situation without losing his girlfriend Katherine.

Boss has the goal of promoting population growth by culling men who don't wish to commit and settle down with their girlfriends. This goal is external to Vincent, and indirectly has motivated him to work to overcome the dreams and his visions of Catherine.

Once Vincent becomes aware of this goal, his new goal becomes to defeat Boss's challenge, which aligns with his original goal of not losing Katherine. He forces Boss to agree to explain the Catherine situation to Katherine.

Internal goal:

External goal: Keep Catherine a secret from Katherine, defeat the nightly dreams, best Boss

Plot-Driven Analysis: 
PD Events are often explanatory - giving more information about the source of the dreams.

They also tend to involve Catherine suddenly appearing in Vincent's life again, causing him turmoil in trying to keep to his internal goal of keeping her a secret from Katherine.

\subsection{To The Moon}

The main characters of the story are Eva and Neil, the memory specialists. Their external goal is to carry out Johnny's wishes to reach the moon.

Internal Goals are slightly unclear for Neil and Eva - this is just a job to both of them. They have differences in ethical views about how far is acceptable to go in order to make this happen. Eva believes deleting his wife River is acceptable, where Neil does not. However, the entire story can be thought of as a result of Johnny's internal desire to go to the moon.

Plot-Driven Analysis:

PD events are generally related to Johnny's blocked memory, which reveal the origin of his desire to reach the moon.

\subsection{Tactics Ogre: Let Us Cling Together}

Denam:

Internal Goal: Freedom for his people

External Goal: Lead the resistance

Catiua:

Internal Goal: Not to lose her brother / be alone 
External Goal: Help Denam with the resistance, briefly help Lanselot when obtaining her birthright in order to get Denam's attention.

Plot-Driven Analysis:

PD Events generally move the pieces around the board with regards to the various factions and their position within the war - changing up the field to provide Denam new challenges between where he is, and freedom for his people.

\subsection{Destiny}

External goal is the Speaker's organization and the Guardians staving off the Darkness and protecting the city.

It's unclear what the Player character's internal motivation is.

Plot-Driven Analysis:

It's unclear what the player's internal goal is, so the PD events therefore do not seem overly related to internal goals. PD Events are at times based on external goals, but the PD events themselves seem to cause the external goals, not always related to what happened previously.

\subsection{Batman: Arkham Asylum}

Internal Goal: Justice, peace for Gotham.

External Goal: Batman's goal is to apprehend the Joker, and retrieve the asylum from his control.

Plot-Driven Analysis: 
PD events are often arbitrary barriers in Batman's quest. Some PD events reveal the backstory behind how the Joker planned the night, which helps Batman plan his retaliation strategy.

Scarecrow Fear Gas episodes give us some insight to Batman's internal thoughts.

\subsection{Legendary}

Throughout the entire game, it's unclear what Deckard's internal goals are.

The external goals are to undo the damage caused by opening Pandora's Box at the beginning of the game.

Plot-Driven Analysis:

PD events are very random and disconnected. Most seem to just be excuses to add levels to the game.

\subsection{Etrian Odyssey 2 Untold}

Arianna's goal is to complete her ritual, because it is her duty. Is this internal or external?

The Player and Flavio are initially given the external goal to assist Arianna. After the Player is branded by The Black Guardian, his goal becomes to grow his power and figure out where it comes from / why he has it.

Bertrand joins the party, with his hidden internal goal of completing the next ritual and making right his absence at the last one. 
Once the Black Guardian reveals that Ginnungagap has been irreversibly damaged, the party's goal shifts to defeating the Calamity permanently. Arianna sees this as an extension of her original goal to complete the ritual.

Internal goals:

Arianna: Carry out her duty

Player: Less clear - to figure out what's going on with their transformation in ginnungagap? Loyalty to Arianna?

External Goals:

Arianna: Traverse the Labyrinth, defeat the Calamity

Player: Traverse the Labyrinth to gain power, defeat the Calamity

Plot-Driven Analysis:

PD Events are usually related to the slow exploration of Ginnungagap, and the Black Guardian. They provide framing and the next objective towards completing the external goals. The external goals are reasonably well related to the internal goals. Some PD events are just excuses for more levels. Some early PD events are later revealed to be tests set forth by the Overlord.

\subsection{Dark}

Internal Goal: Determine what happened to him?

External goal: Drink his creator's blood in order to finish his transformation into a vampire. 
Plot-Driven Analysis:

Most of the game is PD events. Information about Eric / Vampires / Next steps is revealed randomly, out of nowhere. The flow of the game story doesn't seem overly logical - just Eric being sent on a series of missions escalating in difficultly purely because the previous mission did not work. When the plot twist of Eric's identity is revealed, there is little impact because it does not seem related to anyone's internal goals, and Eric's reaction does not seem based on any real experiences / information.

\subsection{Velvet Assassin}

Internal Goal: Very unclear. Perhaps guilt over innocents who are caught up in her missions?

External goal: Complete her missions, escape from enemy hands

Plot-Driven Analysis:

The bulk of the game are PD event dominated missions, which have little to do with Violette's current state. The only events which touch on / imply internal goals are CD. It is only very late in the game once we are in the "current time" where the PD events seem related to what we've been able to infer about Violette's internal goals.

\subsection{Fire Emblem Fates}

This game is unique in the data by being three distinct campaigns that are the result of a single player choice early on in the story.

In all three paths the main character Corrin's internal goal is to bring peace to the two kingdoms. All three paths differ in how Corrin goes about doing this. 


\subsubsection{Birthright}

Corrin chooses to side with her biological family and Hoshido because she cannot condone Garon's actions and believes he has gone mad. She resolves to defeat Garon and end the war.

Internal goal: end war, bridge her two families

External goal: Defeat Garon

Plot-Driven Analysis:

PD Events towards the end center on Nohr's current state as a decaying nation, and provide motivation to try and end the war, not just for Hoshido's sake, but for Nohr as well. Towards the beginning, it's more difficult to see how PD events are related to the internal goal.

\subsubsection{Conquest}

Corrin chooses to side with her adopted family and Nohr with the belief that she can convince Garon to stop his war from the inside.

Internal goal: end war, bridge her two families

External goal: Expose the force controlling Garon so that he can be defeated

Plot-Driven Analysis:

Some PD events are unrelated, such as Elise's fever. Others are related to the force controlling Garon, providing information / proof that there IS a force. Some PD events illustrate the effect of the war on the world, providing Corrin further motivation to end it. 


\subsubsection{Revelations}

Corrin directly tries to ask for peace, and refuses to choose a side, earning her the initial ire of both families.

In this path, the existence of Valla and Anankos' involvement in the war is made known to Corrin early on by Azura.

Internal goal: end war, bridge her two families

External goal: Convince siblings of the true threat, Defeat Anankos

Plot-Driven Analysis:

Many of the PD events are related to Corrin's desire to bridge the gap between her families, and get them to share a common goal (ending the war and defeating Anankos).

\subsection{Shadow of Mordor}

Talion:

Internal Goal: Achieve personal peace with his death and the death of his family

External Goal: Defeat the Black Hand, avenge his family

Celebrimbor:

Internal Goal: Avenge his family's death

External Goal: Defeat Sauron

Plot-Driven Analysis: 
Several PD events are related to the relics which are found throughout the game, which show Talion Celebrimbor's memories. The acquisition of these relics give Celebrimbor power, which furthers his internal goals. Few of the PD events are related to Talion, compared to Celebrimbor.

\subsection{Gravity Rush}

Kat:

Internal Goal: Very unclear.

External Goal: Return the missing parts of town, defeat Anemone?

Raven:

Internal Goal: Save the children / her brother

External Goal: Assist D’Nelica, stop Kat

Raven's goals are much clearer than Kat's. Kat seems to just do whatever is asked of her by everyone, without much of a clear internal driving force. You would think she would be trying to discover who she is and recover her memories, but I don't really get that impression.

Plot-Driven Analysis:

Many PD events are unrelated to anything. Towards the beginning, Kat is just aimlessly running around the town. Some PD events are later revealed to be a result of Raven's internal goals. However, most of the PD events still seem disjointed, and the flow of the story does not seem to be overly based on internal goals. 